\title{
ANTI-TUMOR AND RADIO-SENSITIZING PROPERTIES OF AD-IU2, A PROSTATE-SPECIFIC REPLICATION-COMPETENT ADENOVIRUS ARMED WITH TRAIL
}

Juan Antonio Jiménez

Submitted to the faculty of the University Graduate School

in partial fulfillment of the requirements for the degree

Doctor of Philosophy

in the Department of Microbiology and Immunology, Indiana University

December 2008 
Accepted by the Faculty of Indiana University, in partial fulfillment of the requirements for the degree of Doctor of Philosophy.

Thomas A. Gardner, M.D., Chair

Chinghai Kao, Ph.D.

Doctoral Committee

September 16, 2008

David W. Crabb, M.D.

Maureen A. Harrington, Ph.D.

Ann Roman, Ph.D. 
(C) 2008

Juan Antonio Jiménez

ALL RIGHTS RESERVED 
To my beautiful wife Heather

and precious daughter Sofía,

who have made this part of my life a dream come true. 


\section{Acknowledgements}

Nothing great in life can be accomplished without the help and support of friends and family. Likewise, I could not have arrived at this point in my academic career without the constant and loving support of my parents. They have been shining examples of what can be achieved with hard work and perseverance. Six years ago, I met my soul mate, Heather. Since then, she has been my best friend and my source of strength and comfort. Her unconditional love is a gift, and I look forward to spending the rest of my life with her. Together, we have been blessed with a beautiful daughter, Sofía. With each day that passes, she continues to amaze me as she grows, learns and explores the world around her.

To the members of the Urology Research Laboratory, both past and present, I would like to say thank you. I have learned a great deal from my fellow friends and colleagues. I would like to especially thank a few members without whose assistance I could not have completed my thesis research. Yan-Ping Zhang is truly the go-to person in the lab with all of the answers. She taught me all I ever wanted to know about adenoviral vector production and often provided technical expertise and assistance. During the second portion of my thesis, Yousef Mohammadi provided countless hours of assistance with irradiations, clonogenic assays and flow cytometry. Over the years, I have shared an office with Sang-Jin Lee and later Matt Mellon. Thank you both for all of the good times, great conversations, friendship and advice.

I would like to thank the faculty and staff of the Department of Microbiology and Immunology and the Department of Urology for their time, assistance and support during my Ph.D. years. Likewise, I would like to thank the staff and advisors of the MD/PhD program for providing the structure and resources necessary to complete my training as a physician-scientist. 
I would like to thank each of the members of my thesis committee for contributing time and effort to my education. Over the past four years, you have provided valuable support, guidance and advice.

I would especially like to thank Dr. Tom Gardner and Dr. Chinghai Kao for their guidance, support and mentorship. During my training I was fortunate to train under two brilliant minds. Tom, you have been an exceptional model of a physician-scientist and have taught me the importance of science in the practice of medicine. Chinghai, you have taught me the importance of a well formulated hypothesis, precise scientific technique and the excitement of discovery. Tom and Chinghai, over the past six years, I have enjoyed and appreciated our friendship and conversations, and I look forward to our continued friendship and collaboration.

Finally, I would like to thank the National Institutes of Health and the National Cancer Institute for providing the funding for my thesis research through the Ruth $\mathrm{L}$. Kirschtein National Research Service Award Individual Fellowship 1F31 CA106215-01 awarded September 2003 through September 2008. 


\begin{abstract}
Juan Antonio Jiménez

ANTI-TUMOR AND RADIO-SENSITIZING PROPERTIES OF AD-IU2, A PROSTATESPECIFIC REPLICATION-COMPETENT ADENOVIRUS ARMED WITH TRAIL
\end{abstract}

In this thesis, I investigated the preclinical utility and antitumor efficacy of TRAIL delivered by Ad-IU2, a prostate-specific replication-competent adenovirus (PSRCA), against androgen-independent prostate cancer. Through transcriptional control of adenoviral early genes $E 1 a, E 1 b$ and $E 4$, as well as TRAIL by two bidirectional prostate-specific enhancing sequences (PSES), expression of TRAIL as well as adenoviral replication was limited to prostatespecific antigen and prostate-specific membrane antigen (PSA/PSMA)expressing cells. Ad-IU2 replicated efficiently in and was restricted to PSA/PSMA-positive prostate cancer cells and induced 5-fold greater apoptosis in androgen-independent CWR22rv and C4-2 prostate cancer cells than the PSRCA control not expressing TRAIL. Ad-IU2 exhibited superior killing efficiency in PSA/PSMA-positive prostate cancer cells at doses 5 to 8 -fold lower than that required by a non-TRAIL expressing PSRCA to produce a similar effect. This enhanced cytotoxic effect was not observed in non-prostatic cells, however. As an enhancement of its therapeutic efficacy, Ad-IU2 exerted a bystander effect through either direct cell-to-cell contact or soluble factors present in conditioned media from Ad-IU2-infected cells. In vivo, Ad-IU2, as compared to a control PSRCA, markedly suppressed the growth of subcutaneous CWR22rv xenografts 
at six weeks post-treatment ( $3.1 \mathrm{vs} .17 .1$-fold growth of tumor). The treatment of androgen-independent prostate cancer with Ad-IU2 prior to external beam radiation therapy (EBRT) significantly reduced clonogenic survival with dose reduction factors of 4.91 and 2.43 for CWR22rv and C4-2 cells, respectively. Radio-sensitization by Ad-IU2 was restricted to PSA/PSMA-positive cells. Combinatorial radio-gene therapy resulted in accumulation of cells in $G_{1}$ phase and a perturbation of the radiation-induced $\mathrm{G}_{2}$ phase arrest. This multi-modal approach combining viral lysis, apoptosis-inducing gene therapy, and radiation therapy could have great impact in achieving complete local tumor control while reducing radiation dose and associated treatment morbidities. This would result in improvement of the clinical outcome of patients with high risk prostate cancer.

Thomas A. Gardner, M.D., Chair 


\section{Table of Contents}

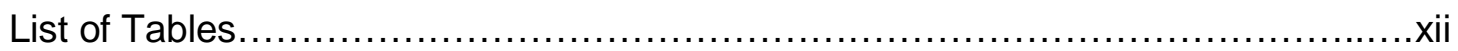

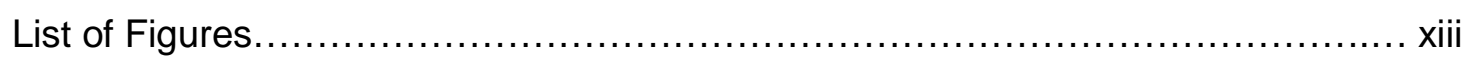

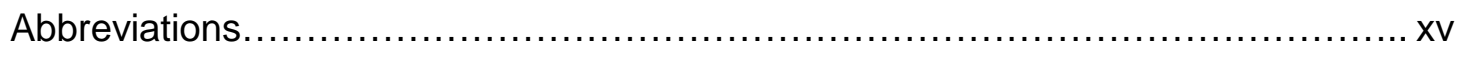

Introduction

Clinical Significance of Prostate Cancer.................................. 1

Molecular Pathogenesis of Prostate Cancer.............................. 4

Current Therapy for Locally Advanced Prostate Cancer.....................6

The Development of Molecular Therapy for Prostate Cancer.............. 9

Gene Delivery Vectors..................................... 9

Prostate- and Tumor-Specific Promoters.......................... 17

Current Gene Therapy Approaches for Advanced Prostate Cancer............22

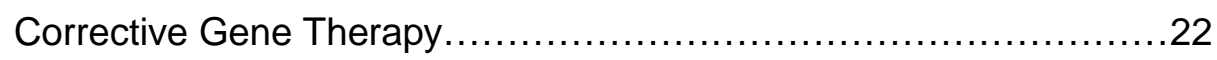

Oncolytic Viral Therapy............................................

Cytotoxic Gene Therapy...................................... 33

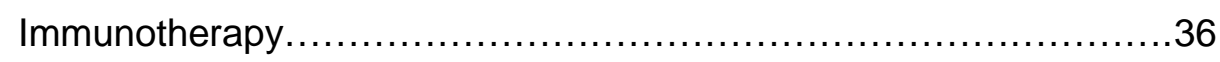

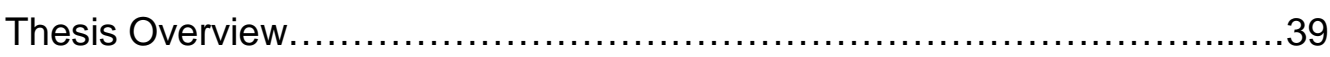

Materials and Methods

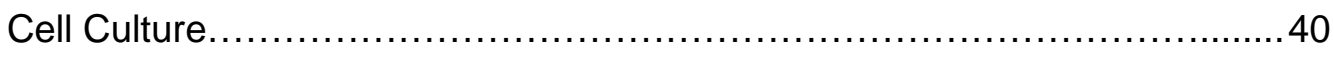

Production of Adenoviral Vectors...................................... 41

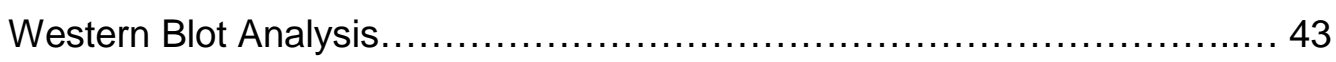

Measurement of TRAIL Surface Expression........................... 44

Viral Replication Assay.............................................. 44

Measurement of Apoptosis Induction.................................. 45

In Vitro Cell Killing Assay.............................................. 45 
Evaluation of Bystander Effect................................................. 46

In Vivo Evaluation of Ad-IU2 Anti-Tumor Effect................................. 47

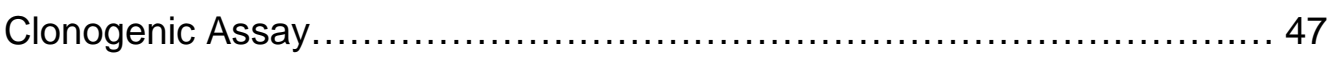

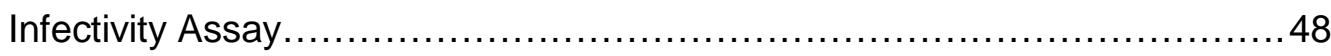

MTT Assay for In Vitro Cell Survival................................................. 49

Measurement of Apoptosis Induction Following Irradiation of Cells............ 49

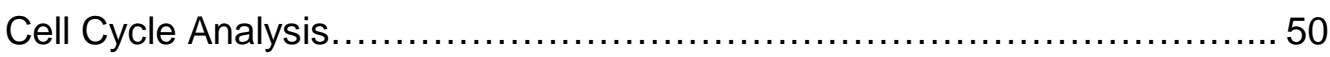

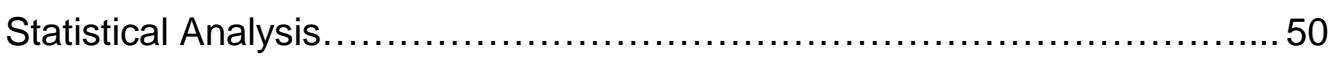

Development of Ad-IU2 for Androgen-Independent Prostate Cancer

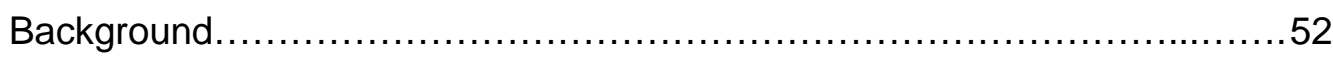

In Vitro and In Vivo Characterization of Ad-IU2 .................................. 55

Ad-IU2 Delivered Surface-Bound TRAIL and Replicated Effectively

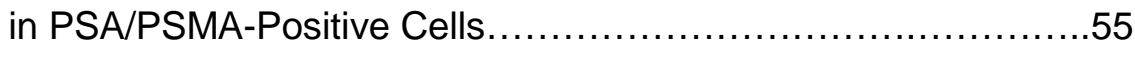

Ad-IU2 Effectively Induced Apoptosis and Reversed Resistance in PSA/PSMA-Positive Prostate Cancer Cells.............................59

Ad-IU2 Effectively Killed Prostate Cancer Cells, While It Spared

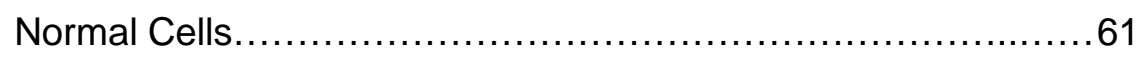

Ad-IU2 Demonstrated a Bystander Effect in PSA/PSMA-Negative

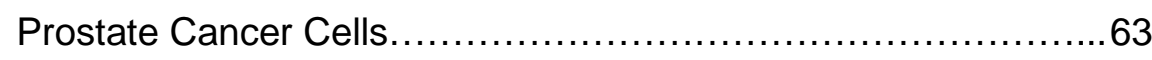

Ad-IU2 Inhibited the Growth of SQ Androgen-Independent

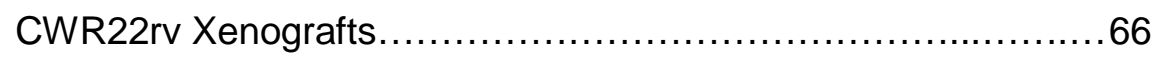

Radio-Sensitization of Androgen-Independent Prostate Cancer with Ad-IU2

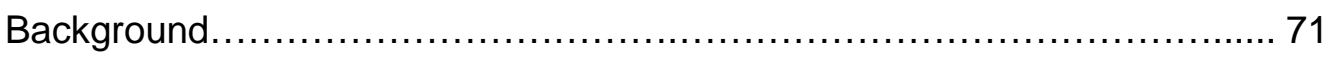

Evaluation of the Combination of Ad-IU2 and Radiation Therapy for the

Treatment of Androgen-Independent Prostate Cancer....................... 74 
Ad-IU2 Reduced the Clonogenic Survival of Irradiated Prostate Cancer Cells More Effectively Than rhTRAIL Alone............... 74

Radio-Sensitization by Ad-IU2 was Specific to PSA/PSMA-Positive

Prostate Cancer Cells.......................................... 80

Combinatorial Therapy Failed to Augment Induction of Apoptosis..... 81

Pretreatment of Androgen-Independent Prostate Cancer Cells with Ad-IU2 or rhTRAIL Resulted in $\mathrm{G}_{1}$ Phase Accumulation and Diminished Radiation-Induced $\mathrm{G}_{2}$ Phase Arrest..............84

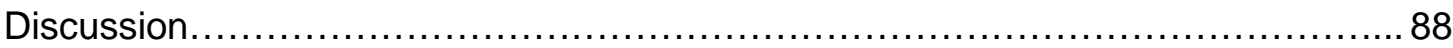

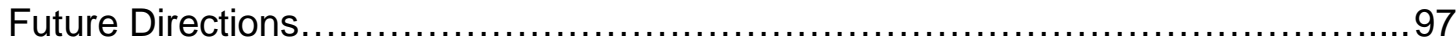

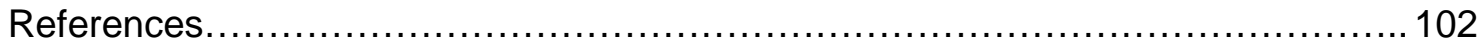

Curriculum Vitae 


\section{List of Tables}

Table 1. Characteristics of vector systems commonly used for molecular

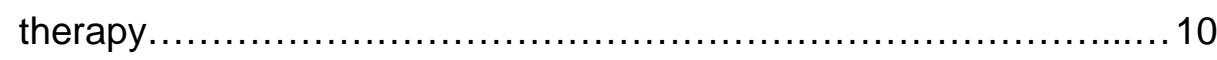

Table 2. Current approaches for prostate cancer gene therapy clinical trials........23

Table 3. Characterization of prostate cancer cell lines used in these studies........41

Table 4. Ad-IU2 replicated efficiently in and restricted to PSA/PSMA-positive

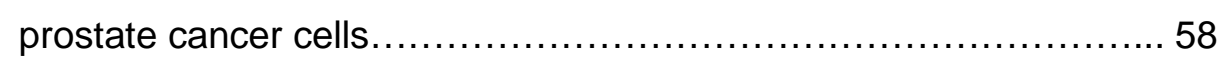

Table 5. Ad-IU2 sensitized CWR22rv cells to EBRT ................................77

Table 6. Ad-IU2 sensitized C4-2 cells to EBRT .......................................79

Table 7. Relative infectivity of LoVo and T24 cells for Ad5 ............................. 81

Table 8. Combination of Ad-IU2 or rhTRAIL and EBRT resulted in perturbation

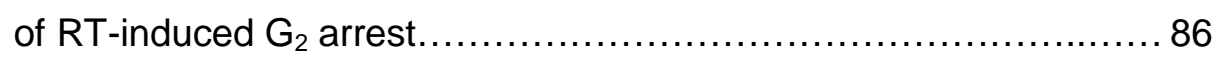




\section{List of Figures}

Figure 1. Molecular pathogenesis and progression of prostate cancer.............4

Figure 2. Organization of the adenoviral genome............................. 11

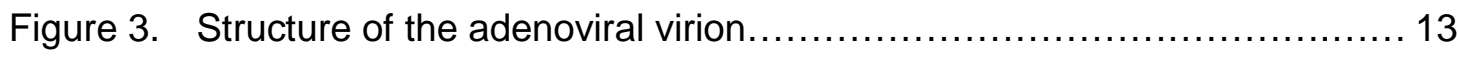

Figure 4. Induction of apoptosis by TRAIL via the extrinsic and intrinsic

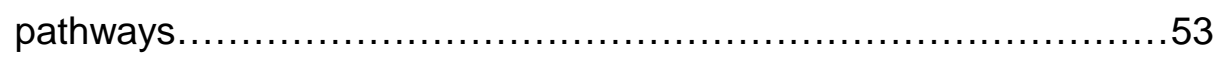

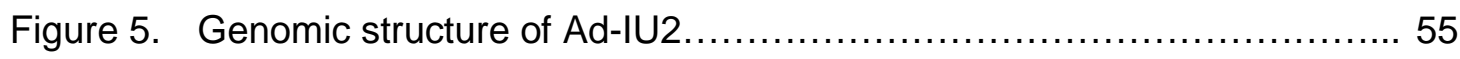

Figure 6. Expression of TRAIL in PSA/PSMA-positive CWR22rv cells was

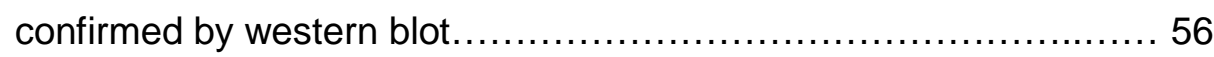

Figure 7. Cell surface expression of TRAIL was confirmed in CWR22rv prostate cancer cells following infection with Ad-IU2 ...............5 57

Figure 8. PSES-driven expression of adenoviral E1a was limited to PSA/PSMA-

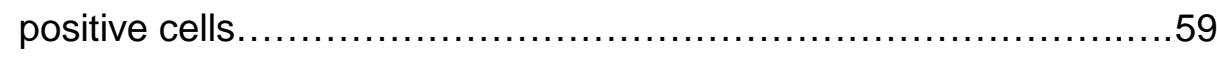

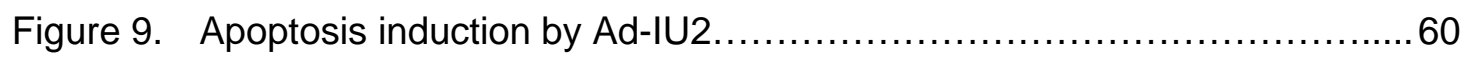

Figure 10. TRAIL resistance was reversed in LNCaP cells following prolonged

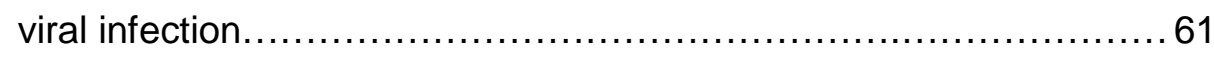

Figure 11. Ad-IU2 induced marked in vitro cell killing of PSA/PSMA-positive prostate cancer cells.............................................. 62

Figure 12. Ad-IU2 demonstrated a bystander effect in PSA/PSMA-negative cells in co-culture with infected PSA/PSMA-positive cells.

Figure 13. Heat-inactivated conditioned media from Ad-IU2-infected CWR22rv cells induced significant levels of apoptosis in PSA/PSMA-negative prostate cancer cells. .65

Figure 14. Heat treatment of conditioned media was sufficient to inactivate adenovirus. 65 
Figure 15. TRAIL was not cleaved from the surface of Ad-IU2-infected CWR22rv cells and present in conditioned medium at physiologically relevant concentrations .66

Figure 16. Ad-IU2 suppressed the growth of androgen-independent human prostate tumors in athymic mice. .67

Figure 17. Individual fold tumor growth at the 6-week study endpoint. 68

Figure 18. Histological appearance of CWR22rv tumors 6 weeks after treatment.....69

Figure 19. In situ detection of apoptosis in CWR22rv xenografts..................... 70

Figure 20. Determination of optimal viral dose for clonogenic assay................75

Figure 21. Combination of Ad-IU2 and RT resulted in a decrease in the clonogenicity of CWR22rv cells. 76

Figure 22. Combination of Ad-IU2 and RT resulted in a decrease in the clonogenicity of C4-2 cells. 78

Figure 23. Adenoviral infectivity assay for LoVo and T24 cells. .80

Figure 24. Ad-IU2 failed to sensitize PSA/PSMA-negative colorectal and bladder cancer cell lines to EBRT..... 82

Figure 25. Combination of Ad-IU2 plus EBRT did not enhance apoptosis induction at doses sufficient to decrease clonogenicity of PSA/PSMApositive prostate cancer cells.

Figure 26. Treatment of CWR22rv and C4-2 cells with Ad-IU2 or rhTRAIL resulted in accumulation of cells in $\mathrm{G}_{1}$ phase. .84

Figure 27. Combination of Ad-IU2 or rhTRAIL and EBRT resulted in perturbation of RT-induced $\mathrm{G}_{2}$ phase arrest. .87

Figure 28. Hypothetical mechanism of radio-sensitization by Ad-IU2 .100 


\section{Abbreviations}

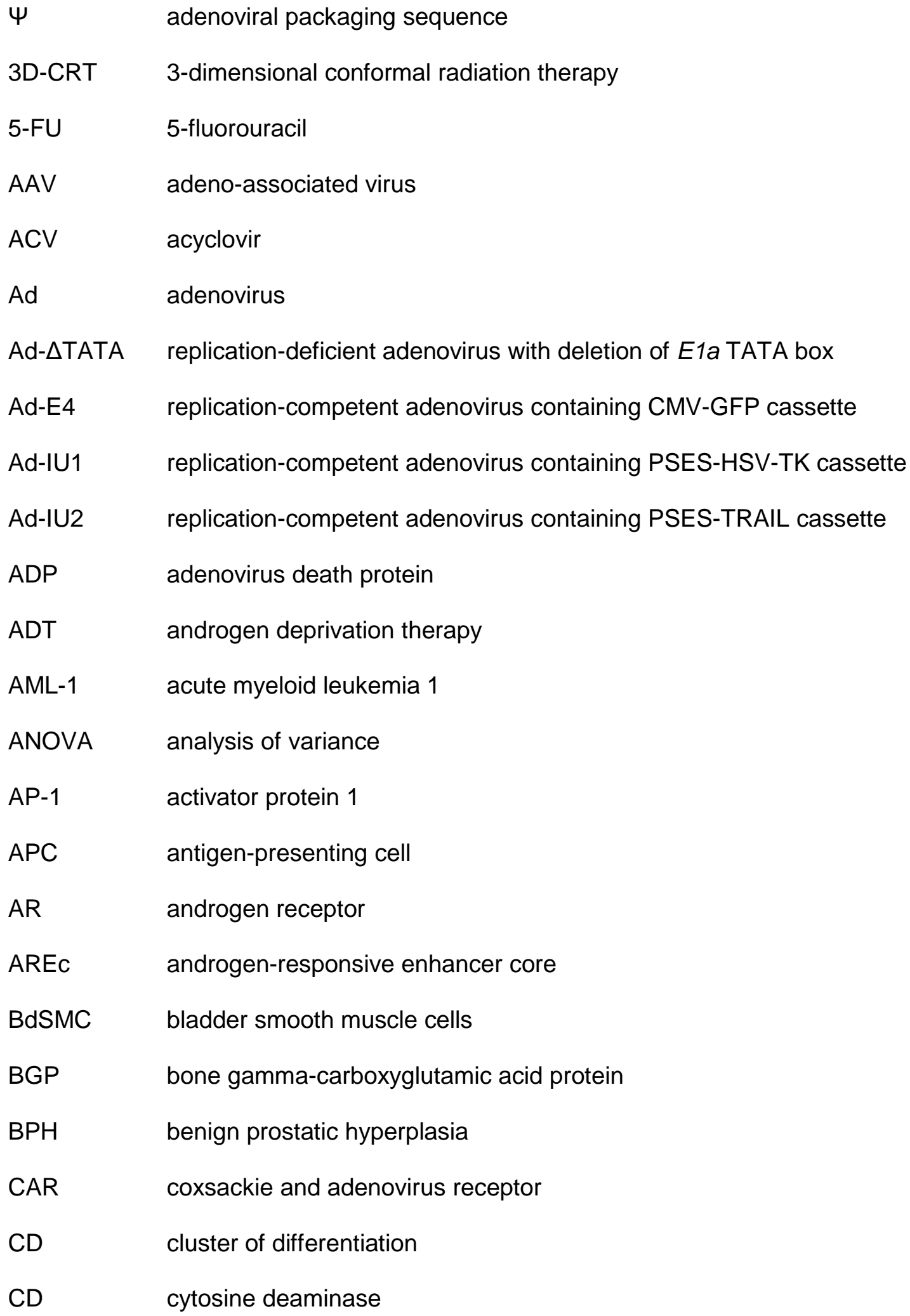




\begin{tabular}{|c|c|}
\hline CDK & cyclin-dependent kinase \\
\hline $\mathrm{CM}$ & conditioned medium \\
\hline CMV & cytomegalovirus \\
\hline CpG & cytidine-phospho-guanonsine \\
\hline CTL & cytotoxic T lymphocyte \\
\hline DC & dendritic cell \\
\hline DcR & decoy receptor \\
\hline DR & death receptor \\
\hline DMEM & Dulbecco's modified Eagle's medium \\
\hline DRF & dose reduction factor \\
\hline DTT & dithiothreitol \\
\hline EBRT & external beam radiation therapy \\
\hline ECL & enhanced chemiluminescence \\
\hline EGFP & enhanced green fluorescent protein \\
\hline ERG & v-ETS oncogene-like \\
\hline ETS & erythroblastosis virus E26 transforming sequence \\
\hline ETV1 & ETS variant gene \\
\hline FACS & fluorescence-activated cell sorting \\
\hline FADD & Fas-associated death domain \\
\hline FasL & Fas ligand \\
\hline FBS & fetal bovine serum \\
\hline GADD45 & growth arrest and DNA damage-induced gene \\
\hline GC & guanine-cytosine \\
\hline GCV & ganciclovir \\
\hline GFP & green fluorescent protein \\
\hline GM-CSF & granulocyte-macrophage colony-stimulating factor \\
\hline
\end{tabular}




$\begin{array}{ll}\text { GnRH } & \text { gonadotropin-releasing hormone } \\ \text { GRE } & \text { glucocorticoid-responsive element } \\ \text { GSTP1 } & \text { glutathione S-transferase P1 polymorphism } \\ \text { Gy } & \text { Gray } \\ \text { HDFa } & \text { adult human fibroblasts } \\ \text { H\&E } & \text { Hematoxylin and Eosin } \\ \text { HEK } & \text { human embryonic kidney } \\ \text { HER } & \text { human embryonic retinoblast } \\ \text { hK3 } & \text { human kallikrein-3 } \\ \text { HLA } & \text { human leukocyte antigen } \\ \text { HPC } & \text { hereditary prostate cancer } \\ \text { hrl } & \text { humanized Renilla luciferase } \\ \text { HRP } & \text { horse radish peroxidase } \\ \text { HPV } & \text { human papillomavirus } \\ \text { HSV-TK } & \text { herpes simplex virus-thymidine kinase } \\ \text { hTERT } & \text { human telomerase reverse transcriptase } \\ \text { HUC } & \text { human urothelial cells } \\ \text { IL } & \text { interleukin } \\ \text { IMRT } & \text { intensity-modulated radiation therapy } \\ \text { INF } & \text { interferon } \\ \text { IND } & \text { investigational new drug } \\ \text { IRES } & \text { internal ribosomal entry sequence } \\ \text { ITR } & \text { inverted terminal repeats } \\ \text { LD } & \text { lethal dose } 50 \% \\ \text { LDU } & \text { LD } 50 \text { units } \\ \text { murine double minute clone 2 } \\ \end{array}$




\begin{tabular}{ll} 
MHC & major histocompatibility complex \\
MLP & major late promoter \\
MUC & mucin \\
mRFP & monomeric red fluorescent protein \\
MTT & (3-(4,5-dimethylthiazol-2-yl)-2,5-diphenyltetrazolium bromide) \\
NFATc1 & nuclear factor of activated T-cells 1 \\
NFKB & nuclear factor-kappa B \\
NIS & sodium iodide symporter \\
NK & natural killer \\
OBA & Office of Biotechnology Activities \\
OC & osteocalcin \\
PAP & prostatic acid phosphatase \\
PBS & phosphate-buffered saline \\
PCSM & prostate cancer-specific mortality \\
PEG & polyethylene glycol \\
PI & propidium iodide \\
PI3K & phosphoinositide 3-kinase \\
PIN & prostatic intraepithelial neoplasia \\
PIP2 & phosphatidylinositol (3,4)-bisphosphate \\
PIP3 & phosphatidylinositol (3,4,5)-bisphosphate \\
PKR & RNA-dependent protein kinase \\
pRB & retinoblastoma tumor suppressor \\
PSA & prostate-specific antigen \\
PSES & prostate-specific enhancing sequence \\
PSMA & prostate-specific membrane antigen \\
PSME & PSMA enhancer \\
\hline
\end{tabular}




$\begin{array}{ll}\text { PSRCA } & \text { prostate-specific replication-competent adenovirus } \\ \text { PTEN } & \text { phosphatase and tensin homologue } \\ \text { RAC } & \text { Recombinant DNA Advisory Committee } \\ \text { RGD } & \text { arg-gly-asp } \\ \text { rhTRAIL } & \text { recombinant human TRAIL } \\ \text { RIPA } & \text { radioimmunoprecipitation assay } \\ \text { RPMI-1640 } & \text { Roswell Park Memorial Institute-1640 medium } \\ \text { RSV } & \text { Rous sarcoma virus } \\ \text { RT } & \text { radiation } \\ \text { SDS-PAGE } & \text { sodium dodecyl sulfate-polyacrylamide gel electrophoresis } \\ \text { SQ } & \text { subcutaneous } \\ \text { TGF- } \beta & \text { transforming growth factor-beta } \\ \text { TNF } & \text { tumor necrosis factor } \\ \text { TP } & \text { terminal protein } \\ \text { TRAIL } & \text { TNF-related apoptosis-inducing ligand } \\ \text { TRAMP } & \text { transgenic adenocarcinoma of the mouse prostate } \\ \text { TBST } & \text { tris-buffered saline Tween-20 } \\ \text { TR } & \text { transrectal ultrasound } \\ \text { ttk } & \text { truncated thymidine kinase } \\ \text { TUNEL } & \text { terminal dUTP nick-end labeling } \\ \text { VA } & \text { virus-associated } \\ \text { VAL } & \text { valacyclovir } \\ \text { VDRE } & \text { vitamin D-responsive element } \\ \text { VEGFR-2 } & \text { vascular endothelium growth factor receptor } 2 \\ \text { virus particles } \\ \text { wild-type }\end{array}$




\section{Introduction}

\section{Clinical Significance of Prostate Cancer}

In 2008, it is estimated that prostate cancer will account for the most new cancer diagnoses, aside from skin cancer, at 186,320 men in the United States and will be the second most common cause of cancer-specific mortality at 28,660 men. The incidence of locally advanced or high risk prostate cancer has decreased since the advent of prostate-specific antigen (PSA) screening; however $9 \%$ of newly diagnosed men will present initially with locally advanced or metastatic disease (1). For American men, the overall lifetime risk of developing clinically-detectable prostate cancer is 1 in 6 ; however, post-mortem examination of men above the age of 80 reveals a prevalence of greater than $80 \%$, indicating that most men die with the disease rather than of prostate cancer. In addition, microscopic foci of disease can be found incidentally in surgical specimens from transurethral resections of the prostate for benign prostatic hyperplasia (BPH); however, approximately $90 \%$ of these lesions remain sub-clinical throughout the entire lifetime of the patient (2). The hallmarks of this disease include an initially indolent and multi-focal cancer, temporary response to androgen depletion by growth suppression and apoptosis, development of androgen-independent tumors and distant metastasis to bone in late stages of the disease, resulting in the demise of the patient.

Significant risk factors for developing prostate cancer include age, race and family history. Prostate cancer is rare in men below the age of 50 , with an incidence of only $1 \%$. The risk of developing cancer of the prostate increases with advancing age, from $20 \%$ in men in their fifties to $70 \%$ in men in their seventies (3). Drastic differences in incidence occur among men of different races as well. Men of Asian descent in China and Japan have an age-adjusted incidence of 2 and 9 per 100,000, respectively, compared to 101 for whites in the United States. The greatest risk of developing 
prostate cancer and disease-specific mortality is among African-Americans, with an ageadjusted incidence of 137 per 100,000 (4). Interestingly, when Japanese men immigrate to the United States, their incidence of prostate cancer increases; however, not to the extent of Caucasian-American men, implicating a role for environmental and lifestyle factors. For example, obesity is associated with higher grade prostate cancer and local or biochemical recurrence following radical prostatectomy $(5,6)$. Furthermore, a study involving 46,786 men found that higher caloric intake was associated with increased risk of metastatic prostate cancer and mortality, especially in young men and men with a family history of prostate cancer (7). Higher risk of advanced prostate cancer was also associated with diets high in red meat, saturated fats, dairy products and beer (8-11). Heredity plays a major role in determining risk for the development of prostate cancer. Nearly $10 \%$ of cases world-wide are attributable to inheritance, while the remainder appear to be sporadic and influenced by other factors (12). Several autosomal dominant and X-linked alleles have been identified through segregation analysis and linked to prostate cancer risk. The best described susceptibility locus, hereditary prostate cancer (HPC1) is found on the chromosomal region 1q24-25 (13). Polymorphic variations of the gene encoding the androgen receptor (AR), specifically polyglutamic repeats (CAG), have been described in which the length of the repeat is inversely proportional to AR transcriptional activity $(14,15)$. Finally, certain polymorphisms of $S R D 5 A 2$, the gene that encodes 5- $\alpha$-reductase-2 which is responsible for activating androgens within the prostate, have been shown to have increased enzymatic activity and linked to a 2-fold increase in risk of prostate cancer and disease progression (16).

The majority of cases of adenocarcinoma of the prostate develop in the peripheral zone, along the posterior aspect of the prostate, which is palpable on digital rectal examination. Spread of the disease occurs by local extension beyond the capsule into the seminal vesicles or the bladder neck. Hematogenous spread occurs primarily as 
osteoblastic lesions to the lumbar spine, femur and pelvis, although dissemination occurs rarely to other organs, including the lungs and brain. Lymphatic spread often precedes osseous metastases and occurs initially in the obturator fossa. Grading of prostatic tumors is critical, as a correlation exists between high Gleason score and poor prognosis (17). Tumors are graded on the basis of glandular pattern and differentiation under low-power magnification and are given a score of 1 for well differentiated glands or 5 for poor differentiation. Because prostatic adenocarcinoma is heterogeneous and multi-focal, the Gleason score is comprised of the sum of the primary and secondary patterns observed. Tumor staging is classified using the TNM system where T represents the size of the primary tumor, $\mathrm{N}$ represents spread to lymph nodes, and $\mathrm{M}$ represents evidence of metastatic spread. T stage ranges from 1 for tumors that cannot be palpated and are incidental findings to 4 for tumors that have spread to adjacent tissues beyond the seminal vesicles. Elevation of PSA can occur with cancer of the prostate; however, this is not a cancer-specific marker, and other conditions such as prostatitis and benign prostatic hyperplasia (BPH) can also elevate this serum marker. Of greater prognostic utility is PSA velocity (rate of change in serum PSA values with respect to time), which predicts the aggressiveness of the cancer (18), and PSA doubling time, which is the best surrogate marker for survival in metastatic disease (19).

Historically, the extent of disease was established on clinical staging alone; however, because most prostate cancer diagnosed today is nonpalpable, more quantitative tests are performed to characterize tumors as low, intermediate or high risk of treatment failure. High risk stratification criteria include patients with a single parameter of cT3 disease, Gleason score $>7$, baseline PSA $>20 \mathrm{ng} / \mathrm{ml}$ or pretreatment PSA velocity $>2 \mathrm{ng} / \mathrm{ml}$ per year (20). Although these criteria aid in clinical decision making for the urologist, the management of prostate cancer remains challenging given 
the fact that as many as $15 \%$ of men with prostate cancer-specific mortality (PCSM) initially may have had low risk prognostic factors (21).

\section{Molecular Pathogenesis of Prostate Cancer}

Most prostate cancers are sporadic in nature and result from numerous somatic mutations, DNA hypermethylations, chromosomal rearrangements and gene amplifications. Accumulation of genomic insults is responsible for progression from normal prostatic epithelium to high-grade prostatic intraepithelial neoplasia (PIN) to malignancy, as outlined in Figure 1. Inflammation plays an early role in the development of prostate cancer, especially high-grade PIN (22), and the reactive oxygen species

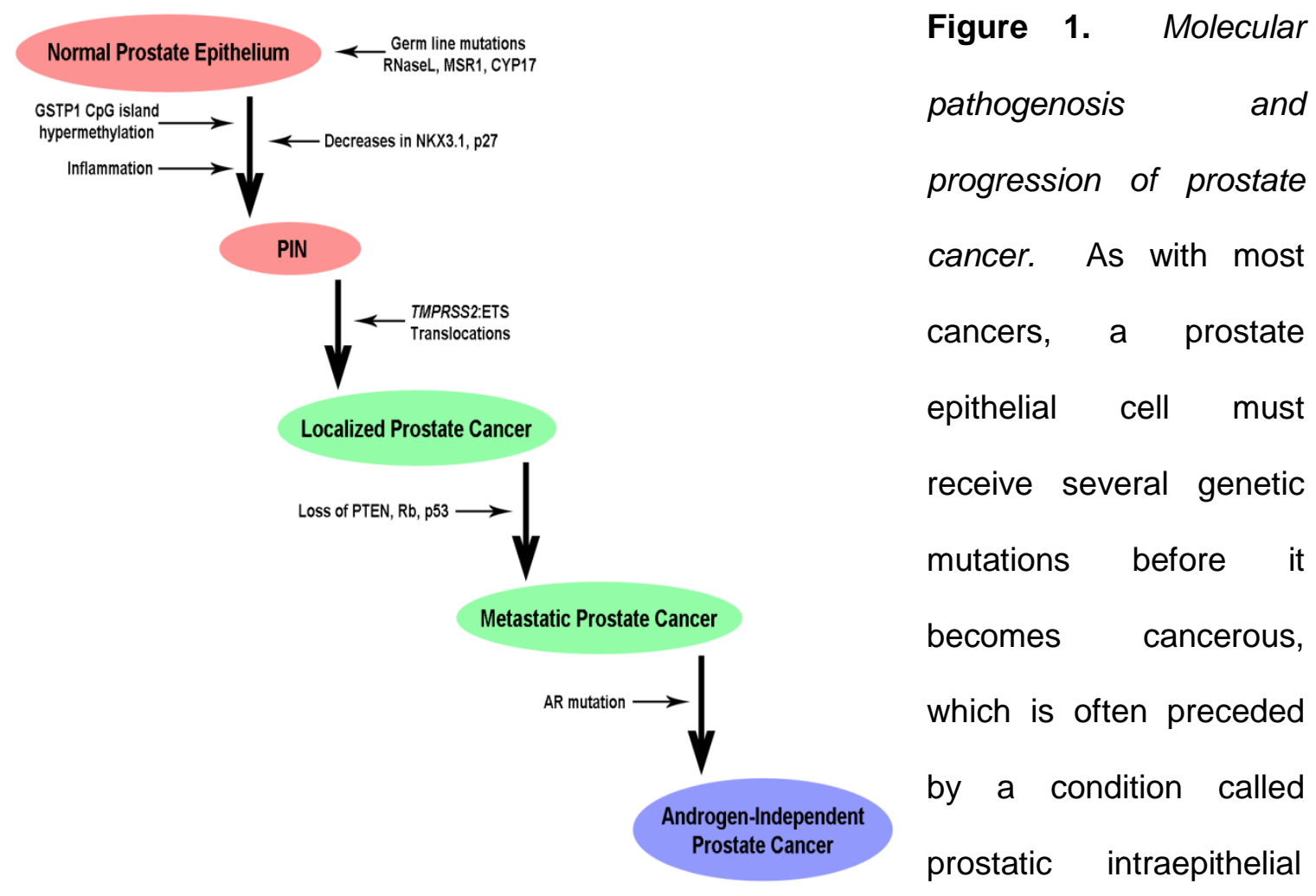

neoplasia (PIN). Aditional mutations are required for a tumor to metastasize and become hormone refractory. 
produced by inflammatory cells cause both cellular and genomic damage. Glutathione S-transferase (GST) is an enzyme produced within the prostate that protects cells against oxidative DNA damage (23), and this house-keeping gene is inactivated in greater than $90 \%$ of cases of PIN and tumors of the prostate by hypermethylation of $\mathrm{CpG}$ islands within the promoter region of $\operatorname{GSTP1}(24,25)$. Loss of chromosome 8p21, and specifically loss of the tumor suppressor gene $N K X 3.1$, occurs early during the progression from high-grade PIN to prostate cancer. This prostate-specific homeobox gene is essential for normal prostate development (26) and represses activity of the PSA promoter (27). Haploinsufficiency of NKX3.1 appears to be correlated to progression of disease, as one study found NKX3.1 expression to be absent in $20 \%$ of PIN lesions, $6 \%$ of $\mathrm{T} 1 \mathrm{a} / \mathrm{b}$ tumors, $22 \%$ of $\mathrm{T} 3 / 4$ samples, $34 \%$ of androgen-independent tumors and $78 \%$ of cancer metastases (28). Recently, chromosomal translocations and gene fusions have been identified involving ETV1 (7p21.2) and ERG (21q22.3), two members of the erythroblastosis virus E26 transforming sequence (ETS) family of transcription factors, and the 5' untranslated region of the prostate-specific transmembrane protease serine 2 (TMPRSS2, 21q22.2) (29). TMPRSS2:ETS fusions act as oncogenes, and because TMPRSS2 is induced by androgen stimulation, ETS genes are overexpressed in prostate cancer, resulting in the progression from PIN and the ability to invade (30). Another tumor suppressor associated with cancer progression is phosphatase and tensin homologue (PTEN), whose somatic mutation was correlated with high grade and stage of prostate cancer (31) and particularly with metastatic disease (32).

Finally, systemic disease is usually treated with androgen deprivation therapy (ADT); however, despite an early response, most advanced tumors will progress to a hormone-refractory state where cell proliferation occurs in the absence of androgens. This occurs through somatic mutations in the gene for AR. Gene amplification allows overexpression of $A R$, and thereby enhances the sensitivity of tumor cells to lower levels 
of circulating androgens (33). Also, somatic mutations within the ligand-binding domain of AR allow constitutive activation or activation by estrogens and anti-androgens (34-36). Treatment of androgen-independent metastatic prostate cancer remains challenging and ultimately leads to poor outcomes.

\section{Current Therapy for Locally Advanced Prostate Cancer}

In two large clinical studies, despite definitive treatment with radical prostatectomy, at least $56 \%$ of patients with cT3 disease experienced biochemical disease recurrence at 10 years, with the development of local and systemic disease recurrence in $27 \%$ of patients $(37,38)$. In a study evaluating the benefit of postoperative irradiation, the addition of adjuvant external beam radiation therapy (EBRT) significantly decreased biochemical failure at 5 years to $26 \%$ vs. $47.4 \%$ compared to the surgery alone group, and the rate of local failure decreased from $19 \%$ to $8.8 \%$; however, because follow-up was only for 5 years, no impact was observed on the rate of distant metastasis formation or PCSM (39). Given that prostate cancer is dependent on activated androgens and the AR for growth (40), adjuvant androgen deprivation therapy (ADT) is well established as standard of care for high risk prostate cancer to slow the growth and dissemination of undetectable residual cancer cells at local or distant sites. Primary hormonal therapy involves surgical castration or medical castration with gonadotropin-releasing hormone $(\mathrm{GnRH})$ agonists such as leuprolide and $A R$ antagonists such as bicalutamide. Nearly all advanced prostate cancers will become hormone refractory within 14 to 20 months of initiating ADT $(41,42)$, and secondary hormonal therapy with ketoconazole and $\mathrm{GnRH}$ antagonists such as abarelix merely provide palliation without survival benefit for this fatal disease phenotype. Most patients die within 3 to 5 years of developing osseous metastases. While there is a pressing 
need to develop better systemic therapies to treat or prevent disseminated disease, there also exists an urgent need to develop more effective local treatment modalities.

EBRT for prostate cancer can take the form of electromagnetic (X-ray) or particle (proton) beams focused directly onto the patient's tumor. Regardless of the mode of EBRT, its therapeutic effect may occur directly from ionization of DNA resulting in lethal strand breakage or indirectly from the interaction between the oxygen radical species produced and DNA or a perturbation of cellular homeostasis regulators which are responsible for control of the cell cycle, DNA repair and apoptosis (43). EBRT can be prescribed as definitive treatment for advanced prostate cancer, where a 70 to 80 Gy total dose is administered to the prostate, seminal vesicles and pelvic lymph nodes in 1.8 to 2 Gy fractions, or as palliative treatment of vertebral osseous metastases that compress the spinal cord, where a 30 Gy total dose is administered in 3 Gy fractions (44). Multiple clinical studies have demonstrated that tumor eradication is directly proportional to the dose of radiation given. Hanks et al recently reported significant improvement in biochemical disease-free survival with EBRT dose escalation in high risk patients with pretreatment serum PSA levels of 10 to $20 \mathrm{ng} / \mathrm{ml}$. Patients receiving $<71.5$, 71.5 to 75.6 or $>75.6$ Gy experienced $19 \%, 31 \%$ and $84 \%$ biochemical free survival, respectively at 8 years. Likewise, increased radiation dose enhanced freedom from distant metastases in this patient population (45). These data confirmed the results from a case-matched retrospective study in which a dose increase from 72 to $>76$ Gy for patients with intermediate to high risk prostate cancer resulted in significant improvement in biochemical disease-free survival, freedom from distant metastases and PCSM (46). However, as the radiation dose is elevated, the incidence of moderate to severe adverse effects also increases. Acute reactions are the result of damage to rapidly dividing mucosal cells and include cystitis, hematuria, dysuria, nocturia (from urinary flow obstruction due to an inflamed prostate), proctitis, diarrhea, rectal bleeding 
and tenesmus. Late or chronic reactions are caused by irreversible damage to slowly proliferating cells such as fibroblasts and endothelial cells and include vascular damage, fibrosis, urethral strictures, impotence, vesicorectal fistulas and spinal cord necrosis (in the case of palliative irradiation) $(47,48)$. To overcome these adverse events, 3dimensional conformal radiation therapy (3D-CRT) was developed to accurately limit and shape the radiation field to the target tissue through the use of image-guided beam alignment. A further enhancement of this technology is intensity-modulated radiation therapy (IMRT), where steep intensity gradients are created within the tumor by modulating the intensity of the radiation beam within each field. Despite these advancements, toxicity to adjacent tissues remains the dose-limiting factor resulting in incomplete tumor eradication and poor clinical outcomes.

Clinical evidence supports the hypothesis that poor local control of the primary tumor leads to the development of distant metastases, increased morbidity and ultimate demise of the patient. Fuks et al. documented a 4-fold increase in the relative risk of developing distant metastases in patients with local failure following pelvic lymph node dissection and $\mathrm{I}^{125}$ brachytherapy compared to the risk in patients without local failure. The relationship between distant metastases and local control was seen regardless of tumor stage or grade. The actuarial freedom from distant metastases at 15 years was $77 \%$ in the 351 patients with local control vs. $24 \%$ in 328 patients with local failure. Most importantly, patients who developed distant metastases despite adequate local control, failed sooner (37 months) than patients who experienced local recurrence (54 months). These data suggest that distant metastases in patients with local control preexisted as micrometastases prior to the initiation of therapy (49). In a larger study involving EBRT without adjuvant ADT, local failure was independently and strongly associated with distant failure. The 10 -year freedom from distant metastases was $77 \%$ in the locally controlled group vs. $61 \%$ in the local failure group. As in the previous study, the median 
time to distant metastasis detection was longer in patients with locally controlled disease (54 months) as compared to patients with local failure (34 months). The initial hazard rate of developing distant metastases was higher in patients with local failure $(3.6 \%$ vs. $3.0 \%$ ), suggesting that a more aggressive and malignant tumor may present with a higher incidence of micrometastases at the time of diagnosis. However, the hazard rate for the development of distant failure increased over time, while the risk of distant metastases decreased in the locally controlled group ( $9.1 \%$ vs. $0.8 \%$ at 12 to 15 years), supporting the theory that persistent local disease may embolize tumor cells to distant sites (50). These studies provide evidence that early and complete eradication of all foci of prostate cancer is necessary to achieve a long-term cure and reduce the need for salvage ADT, which is associated with significant morbidity. Therefore, the combination of a radiation sensitizer with EBRT will allow for RT dose reduction, resulting in less acute toxicity and enhanced therapeutic efficacy, resulting in better patient outcomes.

\section{The Development of Molecular Therapy for Prostate Cancer}

\section{Gene Delivery Vectors}

The antitumor effect of gene therapy for prostate cancer is achieved when a therapeutic gene expression cassette is delivered directly to a target cancer cell by a viral vector. The ideal vector would be specific for prostate cancer cells, have high transduction efficiency and be nonmutagenic. Furthermore, a vector should be inexpensive to produce and administer to the patient. Much research is underway to improve the specificity, transduction efficiency, and safety of gene therapy vectors. The route of administration of the therapeutic agent depends by and large on the vector employed. At the present time, most prostate cancer gene therapy trials entail the intralesional administration of the vector, which is quite suitable given the ease with which to visualize the prostate using transrectal ultrasound (TRUS) and its convenient 
transrectal or transperineal access. Ultimately, the desired route of administration is intravenous infusion of the vector. This approach would seek and destroy all foci of cancer, regardless of their location; however, limiting factors include vector half-life, immunologic clearing of the vector and the infection of non-target organs. Recent improvements in prostate-specific promoter systems and viral targeting have allowed this approach to be used in human prostate cancer clinical trials. The viral vectors currently available for use in gene therapy clinical trials are listed in Table 1; however, only adenoviral vectors will be discussed, as this is the viral vector currently under development in our laboratory.

Table 1. Characteristics of vector systems commonly used for molecular therapy. This table lists the key attributes of the vectors used previously in gene therapy clinical trials for prostate cancer.

\begin{tabular}{|c|c|c|c|c|}
\hline & Adenovirus & $\begin{array}{c}\text { Adeno- } \\
\text { associated } \\
\text { virus }\end{array}$ & Retrovirus & $\begin{array}{c}\text { Vaccinia } \\
\text { virus }\end{array}$ \\
\hline $\begin{array}{l}\text { Maximum insert size } \\
\text { In vivo gene delivery } \\
\text { Gene expression } \\
\text { Cell cycle dependent } \\
\text { Genome integration } \\
\text { Immunoreactive }\end{array}$ & $\begin{array}{l}10-35 \mathrm{~kb} \\
\text { High } \\
\text { Transient } \\
\text { No } \\
\text { No } \\
\text { Yes / No }\end{array}$ & $\begin{array}{l}2.4 \text { / } 4.8 \mathrm{~kb} \\
\text { High } \\
\text { Stable } \\
\text { No } \\
\text { Site-specific } \\
\text { No }\end{array}$ & $\begin{array}{c}8 \mathrm{~kb} \\
\text { Low } \\
\text { Stable } \\
\text { Yes } \\
\text { Random } \\
\text { No }\end{array}$ & $\begin{array}{c}>30 \mathrm{~kb} \\
\text { High } \\
\text { Transient } \\
\text { No } \\
\text { No } \\
\text { Yes }\end{array}$ \\
\hline
\end{tabular}

Adenovirus. The most commonly used viral vector in human gene therapy clinical trials is adenovirus, a non-enveloped, icosahedral virus containing a doublestranded linear DNA genome $36 \mathrm{~kb}$ in size. This non-integrating virus has a wide safety profile and is advantageous over many vectors in part due to its ability to carry a large insert and infect any cell, regardless of its cell cycle status. In addition, its genome is 
easily manipulated in the laboratory, and it is readily produced in high titers with relatively minimal expense. Early-generation adenoviral vectors induced strong innate and adaptive immune responses, thereby limiting their gene delivery potential (51). Furthermore, it has been shown that nearly all humans have developed humoral immunity to adenovirus due to previous exposure; however, only $55 \%$ of the detected immunoglobulins are neutralizing antibodies (52). Recently, adenoviral vectors have been modified to reduce immunogenicity, reinstating their hope for clinical utility (53).

The adenoviral genome, as depicted in Figure 2, is flanked on both ends by short inverted terminal repeats (ITRs) which contain identical origins of replication. Near the 5' end is a short packaging signal $(\Psi)$ which is required for proper association of the viral genome with the capsid proteins (54). Its transcription units are divided into two groups, early and late genes. The viral genome carries six early units (E1a, E1b, E2, E3 and

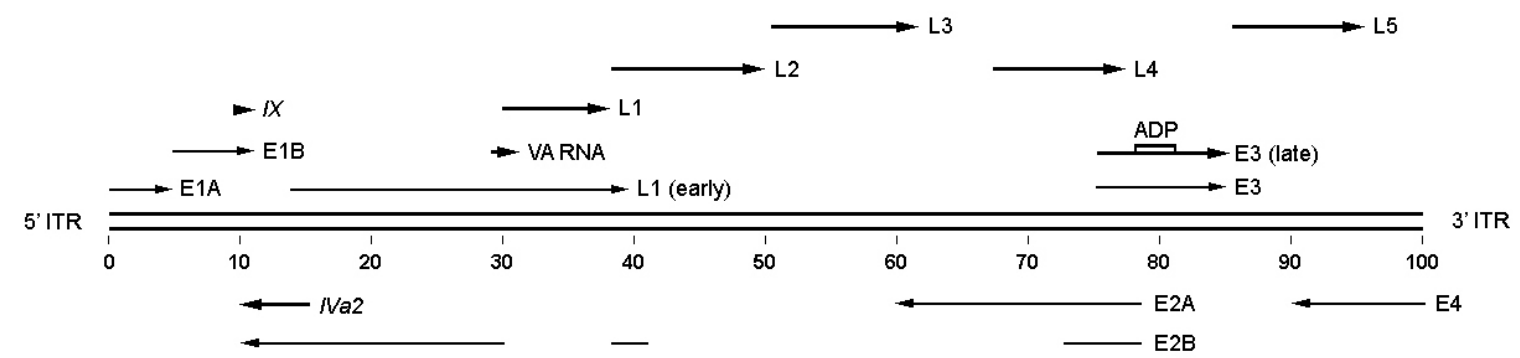

Figure 2. Organization of the adenoviral genome. Adenovirus contains a doublestranded genome that is $36 \mathrm{~kb}$ in length. Transcription occurs from both ITRs and uses alternative splicing to take advantage of overlapping sequences. This transcription map depicts the early adenoviral transcription units with thin arrows, late transcripts in heavy arrows, and delayed mRNAs in italics. Encoded within the E3 region and under the control of the major late promoter is a late transcript which includes adenovirus death protein (ADP). Map units are indicated by the hashes. Products of the late transcripts contribute to the structure of adenovirus. 
E4), two delayed early units (IVa2 and IX), and two late units, one that is processed into five mRNAs (L1-L5) and one in the E3 region that is controlled by the major late promoter (MLP). In addition, either one or two copies of virus-associated (VA) genes are encoded and transcribed by RNA polymerase III. As shown in Figure 2, the transcription of adenoviral genes occurs from both strands and uses alternative splicing and multiple poly $(A)$ sequences.

E1a is the first sequence transcribed in the adenoviral genome. Controlled by a constitutively active promoter, the E1a transcription unit encodes up to five polypeptides, of which only two, E1a $12 \mathrm{~S}$ and E1a 13S, have known functions. E1a proteins transactivate the promoters of other adenoviral genes (55). Expression of E1a is critical to the virus. In fact, deletion of the $E 1$ region of the genome results in a replicationdeficient adenovirus (56). E1a proteins also stimulate viral DNA synthesis by preventing $\mathrm{G}_{1}$ arrest and advancing the host cell into S-phase. This is accomplished by binding pRB and releasing the associated transcription factor E2F (57), antagonizing the cyclindependent kinase inhibitory protein $\mathrm{p} 27_{\mathrm{kip} 1}$ (58), or inhibiting the transactivation of $p 53$ by p300/CBP (59). Furthermore, E1a proteins induce apoptosis of infected cells through p53-dependent and p53-independent pathways (59), both of which are blocked through the action of E1b proteins (60). Acting in concert, E1b and E4 proteins shut-down host cell protein synthesis by blocking the cytoplasmic accumulation of cellular mRNAs, while stabilizing and exporting viral mRNAs from the nucleus to the cytoplasm $(61,62)$. Although adenovirus is not responsible for human malignancies, the products of the E1a, E1b and E4 genes transform cells in vitro (63); however, Ad5, the adenoviral serotype used mostly in prostate cancer gene therapy belongs to a non-oncogenic subgroup. Viral DNA synthesis occurs as E2 gene products accumulate. The $E 2$ region encodes a DNA polymerase that is essential for viral DNA replication (64) and the terminal protein (TP) which is covalently bound to the 5' ends of the viral chromosome and serves as a 
primer for DNA synthesis (65). The expression of $E 3$ is not essential for viral replication; however, it protects virally infected cells from lysis by cytotoxic T-lymphocytes (CTLs) by downregulating the expression of MHC class I antigen (66) and Fas receptor (67) on the infected cell surface. Further protection from the body's antiviral defense system is provided by VA RNA which forms a hairpin-loop structure and inhibits the activation of interferon-induced RNA-dependent protein kinase (PKR) (68).

Late gene products include ten structural proteins, of which seven (II, III, IIIa, IV, $\mathrm{VI}$, VIII and IX) form the capsid and two (V and VII) are involved with the DNA-containing core. Figure 3 depicts the structure of the adenovirus particle. The most abundant protein on the capsid surface is the trimeric hexon (II), whose assembly requires the assistance of the L4 100-kd scaffold protein (69). Neutralizing antibodies to the capsid

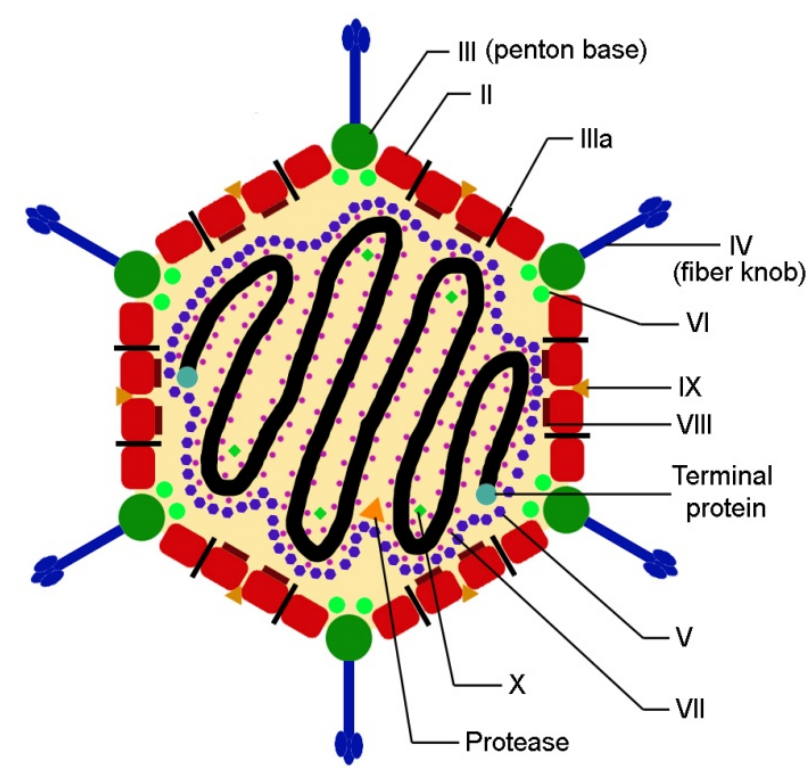

Figure 3. Structure of the adenoviral virion. Adenovirus is a non-enveloped icosahedral virus that is $1,500 \AA$ in diameter. Projecting from the vertices of the virus are twelve fiber knobs, which aid in host cell entry. Proteins II, III, IIIa, IV, VI, VIII and IX form the viral capsid. Proteins V, VII, X and TP associate with the adenoviral genome

and form the viral core. Protease is responsible for the maturation of proteins IIla, VI, VII, VIII and TP.

are formed against surface loops of the hexon structure. Polypeptides IIIa, VI, VIII and IX stabilize the hexon capsid structure and form a bridge with the adjacent core proteins. 
The penton base (III) forms at the twelve vertices of the capsid structure, with a trimeric fiber and distal knob (IV) projecting from each base. Together, polypeptides III and IV form the penton complex and mediate host cell binding. Initially, the knob domain of polypeptide IV binds to its cellular receptor, the coxsackie and adenovirus receptor (CAR) (70), followed by binding of an arg-gly-asp (RGD) motif in the penton to $\alpha_{v} \beta_{3}$ and $\alpha_{v} \beta_{5}$ integrins on the cell surface, stimulating internalization (71). The E3 region encodes one late protein called adenovirus death protein (ADP) which is controlled by the major late promoter and is responsible for cell lysis and release of viral progeny (72). Recently, vectors have been constructed that overexpress ADP, leading to enhanced viral spread and oncolysis (73).

While the majority of prostate cancer cells upregulate CAR expression (74), actual tumors may vary in adenoviral susceptibility. For this reason, efforts have been made to retarget the virus, thereby enhancing its ability to infect prostate cells. Furthermore, the retargeting of adenovirus has narrowed its expansive tropism, allowing for safer systemic delivery without infection of non-target tissues such as liver and respiratory epithelium. Early studies in adenoviral retargeting employed bispecific antibodies which cross-linked the virus to alternative cellular receptors $(75,76)$. This has been applied to prostate cancer with a bifunctional antibody to the adenoviral fiber knob and prostate-specific membrane antigen (PSMA) (77). Perhaps a more clinically feasible approach to prostate-specific retargeting of adenovirus is the genetic modification of the fiber knob. Cell-binding peptides have been displayed on the carboxyl terminus of the fiber knob (78); however, this approach is unfavorable due to size constraints and structural hindrance. Larger peptides can be incorporated into the $\mathrm{HI}$ loop of the fiber knob without structural consequences (79). Lupold et al. identified two candidates for prostate-targeting peptides by phage display library which bind to PSMA (80); however, there exist no reports of its use for the transductional targeting of 
adenovirus. A final approach to the retargeting of adenovirus was devised by Shayakhmetov et al. in which the Ad5 fiber was replaced by the short-shafted Ad35 fiber (81). This chimeric Ad5/35 uses CD46 as its cellular receptor (82) which is upregulated on the surface of many cancer cells. Most importantly, systemic administration of these vectors does not result in hepatic infection, and Ad5/35 has demonstrated prostate cancer tropism (83). In combination with transcriptional targeting, the transductional retargeting and detargeting of adenoviral vectors will increase the safety and efficacy of systemically-delivered molecular therapies for prostate cancer.

Gutless adenovirus. Although adenoviral vectors are attractive gene transfer agents, the presence of adenoviral genes within infected cells triggers an adaptive cellular immune response which produces cytotoxic T lymphocytes (CTLs), resulting in the elimination of transduced cells and shorter transgene expression (84). To overcome this, gutless or helper-dependent adenoviral vectors were developed. These highcapacity vectors (up to $36 \mathrm{~kb}$ insert size) are devoid of all adenoviral coding sequences, resulting in little to no CTL immune response and long-term transgene expression. Therefore, this vector serves best for corrective gene therapy, immunotherapy and the delivery of anti-angiogenic factors. Because gutless vectors only retain the 5' and 3' ITRs and the packaging signal, genomic space not occupied by the therapeutic expression cassette must be filled with stuffer DNA from human introns and non-coding sequences $(85,86)$. To allow for gutless adenoviral genome replication, capsid formation and packaging, wild-type proteins must be provided in trans by co-infection of packaging cells with both a gutless and helper adenovirus. However, the packaging efficiency of the helper virus must be reduced to prevent contamination of the gutless vector sample with replication-competent adenovirus. This was achieved by Parks et al. through the use of a packaging signal flanked by two loxP sequences. When amplified 
in 293Cre cells, a packaging cell line that expresses the Cre recombinase, the packaging sequence is excised, preventing encapsidation of the helper genome (87). Employing this system, contamination levels of 0.1 to $10 \%$ are achieved, which is not acceptable for clinical applications. One possible mechanism for such a high level of contamination is homologous recombination between the helper vector and adenoviral E1 sequences in HEK293 cells. To circumvent this, PER.C6 packaging cells, in which no recombination occurs, can be used for co-infection to achieve contamination levels under $1 \%$ (88). Currently, due to multiple and tedious vector co-infections, large-scale gutless virus production efficiency is limited. This must be improved before helperdependent vectors can be widely deployed for therapeutic applications.

Adeno-associated virus. Adeno-associated virus (AAV), a member of the parvovirus family, is a small single-stranded DNA virus that is dependent on a helper virus, such as adenovirus, for replication. AAV is an attractive vector for gene therapy because it elicits nearly no immune response, is known to cause no disease in humans, and integrates stably and site-specifically in a region on chromosome 19 (89). The genome consists of two ITRs, which encode a packaging signal and the origin of replication, and two genes, rep and cap. Cap encodes the viral capsid proteins while rep encodes four products of alternative splicing, Rep40, Rep52, Rep68 and Rep78, of which only Rep68 or Rep78 are necessary for replication and site-specific integration (90). Recombinant AAV vectors are made by deleting rep and cap and inserting $\sim 4.7 \mathrm{~kb}$ of therapeutic DNA; however, it has been observed that deletion of rep results in nonspecific integration of the virus (91). Nonetheless, the majority of AAV vectors used today are rep-deleted. Recombinant AAV is produced in HEK293 cells by cotransfecting a plasmid containing the therapeutic DNA cloned between two ITRs and a plasmid containing the rep and cap genes. Subsequently, the cells are infected with 
adenovirus or transfected with a plasmid containing the adenoviral genes required for AAV replication (92). Recently, methods have been devised to produce higher AAV titers, necessary for large-scale production for gene therapy applications (93). Following infection, the rate-limiting step in gene expression appears to be the synthesis of the second strand (94). This has been overcome by the recent development of selfcomplementary, double-stranded AAV vectors which carry half the insert size of the single-stranded virus (95). Similar to adenovirus, peptides can be inserted into the AAV capsid to retarget the vector specifically to receptors on the surface of prostate cancer cells (96). To achieve site-specific integration of a transgene with the targeting capability of an adenoviral vector, Recchia et al. developed a hybrid Ad/AAV vector which carries a drug-inducible rep expression cassette and a transgene cassette flanked by two AAV ITRs. Site-specific integration of the ITR-flanked transgene cassette was observed in a rep-dependent fashion (97).

\section{Prostate- and Tumor-Specific Promoters}

Recently, much effort has been made to develop tissue-specific delivery systems that reduce the risk of harming the patient. Several studies have demonstrated the importance of tissue-specific vectors, revealing systemic toxicity with the administration of high doses of nonspecific vectors $(98,99)$. Viral vectors with broad tropisms can transduce any cell in the body, provided that the cell expresses the correct receptor for the virus. Through the use of tissue-specific promoters and enhancers, the expression of a therapeutic gene can be limited to prostate cells. To date, several prostate-specific genes have been identified and their promoters have been well characterized. Because no promoter is prostate cancer-specific, the entire prostate epithelium is susceptible; however, this poses no risk, as the prostate is a non-vital organ in the post-reproductive population. 
Prostate-specific antigen promoter. PSA is released into the bloodstream when the prostatic basement membrane is compromised, such as occurs in prostate cancer and therefore is used as a sensitive serum marker for the diagnosis and progression of prostate cancer (100). PSA, a serine protease, is encoded by the human kallikrein-3 ( $h K 3$ ) gene (101). PSA expression is AR-dependent, and its transcript levels are significantly reduced in the absence of androgen (102). AR regulates PSA expression by binding to a $440 \mathrm{bp}$ androgen-responsive enhancer core (AREc) in the upstream 5' flanking region of the PSA gene $(103,104)$. In vitro experiments have confirmed the tissue-specificity and androgen-dependency of this promoter (105). In addition, this promoter has been used in multiple gene therapy studies $(106,107)$. Although this promoter confers high tissue-specificity, its utility in men undergoing androgen ablation therapy is limited. To circumvent this problem, Gotoh et al. characterized the long (5,837 bp) PSA promoter as less dependent on androgen and therefore, more active than the short (631 bp) PSA promoter in the absence of androgen (106). Two cis-acting elements within the long PSA promoter, a 440 bp AREc and a 150 bp $\mathrm{pN} / \mathrm{H}$ androgen-independent positive regulator, are responsible for this androgenindependent activity. A chimeric promoter with three-fold higher activity than the native PSA promoter has been produced by juxtaposing both elements (108). Further attempts to enhance the activity while retaining the specificity of the PSA promoter in adenoviral vectors include duplication of the AREc, which led to a twenty-fold increase (109) or tandem duplication of the PSA promoter, which led to a fifty-fold increase above basal promoter activity (110).

Prostate-specific membrane antigen promoter. PSMA was discovered by Horoszewicz et al. by a monoclonal antibody produced in mice immunized with cell membranes from LNCaP cells (111). PSMA is a type II integral membrane glycoprotein 
with folate hydrolase (112), N-acetylated alpha-linked acidic dipeptidase (113) and glutamate carboxypeptidase (114) activities. PSMA is expressed predominantly in prostate tissue and tumor neovasculature, with low levels detected in the gastrointestinal tract, salivary glands, kidney and brain (115). Its expression is elevated higher in prostate cancer than in benign hyperplastic or normal prostate epithelium (116). In addition, serum PSMA levels are highest in patients with metastatic disease, suggesting enhanced PSMA expression as prostate cancer progresses (117). Unlike PSA, PSMA expression is upregulated under androgen-depleted conditions (118). A $1.2 \mathrm{~kb}$ PSMA promoter has been identified with high promoter activity (119); however, significant nonspecific activity in PSMA-negative cells limits its clinical usefulness as a gene therapy promoter. Recently, the PSMA enhancer (PSME) was discovered within the third intron of the PSMA gene, FOLH1 (120). Lee et al. have demonstrated PSMA activity mediated by NFATc1 cooperatively binding at the AP-3 site within PSME (121). PSME has been used to transcriptionally target suicide (122) and oncolytic (123) gene therapies for prostate cancer under low androgen levels.

Prostate-specific enhancing sequence. To achieve the highest transcriptional activity with strong prostate-specificity, Lee et al. developed a novel chimeric promoter, called PSES, under the hypothesis that AREc and PSME could function synergistically in any androgen environment. Through deletion and linker scan mutagenesis, the main prostate-specific enhancer activities of the PSA AREc and PSME were located in a 189 bp region called AREc3 and a 331 bp region called PSME(del2), respectively. PSES was developed by combining both AREc3 and PSME(del2) and placing AREc3 upstream from PSME(del2). AREc3 contains six GATA transcription factor binding sites and three AR binding sites, leading to high enhancer activity once surrounding silencer regions were deleted. PSME(del2) contains eight AP-1 and three AP-3 binding sites 
acting as positive regulators in the absence of androgen and a downstream deletion of an Alu transcription-silencing repeat. PSES showed significantly stronger transcriptional activity than either AREc3 or PSME(del2) alone in the presence or absence of androgen. Furthermore, PSES demonstrated five-fold higher activity than universal promoter RSV and activity equal to CMV promoter. In vitro studies revealed that PSES is active in several PSA- and PSMA-positive prostate cancer cell lines, but not in PSA- and PSMAnegative prostate cells or non-prostate cell lines (124). Due to its small size, high level of tissue-specificity, and strong promoter activity regardless of androgen status, PSES is an ideal promoter for use in prostate cancer gene therapy.

Osteocalcin promoter. Osteocalcin (OC) is a highly conserved bone gammacarboxyglutamic acid protein (BGP) that has been shown to be transcriptionally regulated by 1,25-dihydroxyvitamin $D_{3}$ (125). This noncollagenous bone protein constitutes $1-2 \%$ of the total protein in bone, and its expression is limited to differentiated osteoblasts and osteotropic tumors, especially primary and metastatic prostate cancer (126). The osteoblastic nature of osseous prostate cancer metastases is well characterized (127), and the mechanism is believed to be via its osteomimetic properties, specifically its ability to express bone-related proteins such as OC (128). The human OC promoter contains numerous regulatory elements including a vitamin Dresponsive element (VDRE), making it inducible by vitamin $D_{3}$ administration $(129,130)$, a glucocorticoid-response element (GRE), an AP-1 binding site (131), and an AML-1 binding site which has been shown to be responsible for $75 \%$ of $O C$ expression (132). The OC promoter retained its tissue-specificity in a recombinant OC promoter-driven herpes simplex virus-thymidine kinase (HSV-TK)-expressing adenoviral vector. Ko et al. developed a gene therapy for osteosarcoma in which co-administration of Ad-OC-TK and acyclovir (ACV) resulted in osteoblast-specific cell toxicity (133). A similar strategy 
was developed for the intralesional injection of Ad-OC-TK to osseous prostate cancer metastases followed by administration of valacyclovir (VAL). In phase I clinical trials, this therapy induced apoptosis in every lesion treated, without serious adverse effects to the patients $(134,135)$.

Human telomerase promoter. Telomeres are tandem repeat structures found at the termini of chromosomes that maintain chromosomal integrity by preventing DNA rearrangements, degradation and end-to-end fusions. In most normal somatic cells, the telomeric cap is shortened with each cycle of DNA replication and cell division. When telomeres shorten to a critical length, cells progress toward irreversible arrest of growth and cellular senescence (136). In contrast, tumor cells have evolved a means to prevent telomere shortening through the activation of the catalytic component of human telomerase reverse transcriptase (hTERT) (137). The hTERT promoter region has been cloned and characterized, and contains a high GC content. Unlike most promoters, it does not contain TATA or CAAT boxes (138). Importantly, the hTERT promoter is active in most cancer cells including prostate cancer (139) and inactive in most normal cells, thereby providing a unique approach to specifically targeting cancer cells. Promising results have been reported using the hTERT promoter to deliver TRAIL (140) and Bax (141), inducers of apoptosis, to prostate cancer cells. Recently, the hTERT promoter was used to control adenoviral genes $E 1 a$ and $E 1 b$ to control the replication of an oncolytic Adenovirus in a tumor-specific manner. This virus replicated efficiently in and killed a broad spectrum of cancer cells without harming normal human cells lacking telomerase activity (142). Clinical use of this promoter may be limited, however, to local intralesional gene therapy, because systemic delivery of an hTERT virus could have toxic effects on normal proliferating cells and stem cells, in which telomerase is active. 


\section{Current Gene Therapy Approaches for Advanced Prostate Cancer}

The ideal therapy for hormone-refractory prostate cancer would be one that kills cancer cells, but spares normal cells. This therapy would have limited toxicities, increase survival, and enhance the quality of life of men afflicted with prostate cancer. Due to its prior success in clinical studies, gene therapy promises to answer these challenges. While locally advanced prostate cancer can cause much suffering, mortality is usually secondary to the inability to inhibit metastatic spread. Lack of successful conventional therapies for locally advanced and metastatic prostate cancer makes this patient population an excellent candidate for gene therapy. Of course, as with most therapies, the ability to treat men with low volume disease should enhance the success of the therapy.

Over the past decade, four categories of gene therapy approaches for prostate cancer have emerged: corrective gene therapy, oncolytic viral therapy, cytotoxic gene therapy and immunotherapy. Each molecular therapy has a strong foundation of preclinical data allowing for the approval of several clinical studies. Currently, 88 gene transfer protocols registered with the Office of Biotechnology Activities (OBA) are targeted against prostate cancer. This accounts for $15 \%$ of all cancer gene therapy protocols listed to date (143). Table 2 summarizes the approaches registered with the OBA and the Recombinant DNA Advisory Committee (RAC).

\section{Corrective Gene Therapy}

This approach repairs inherited or acquired genetic defects that give the cancer a survival advantage such as those affecting tumor suppressors or growth-promoting oncogenes. These mutations affect the regulation of the cell growth cycle and are among the multiple mutations that occur in the pathogenesis and progression of prostate cancer. Correction of a single genetic insult, however, may not be sufficient to change 
Table 2. Current approaches for prostate cancer gene therapy clinical trials. This list includes all clinical trials listed on the Office of Biologic Activities (OBA) protocol list as of February 29, 2008 (143).

\begin{tabular}{|c|c|c|}
\hline Strategy & Vector & Transgene \\
\hline \multirow[t]{2}{*}{ Corrective } & Adenovirus & $\begin{array}{l}\text { p16 } \\
\text { p53 }\end{array}$ \\
\hline & Retrovirus & c-myc antisense \\
\hline \multicolumn{3}{|l|}{ Cytoreductive } \\
\hline (suicide) & Adenovirus & $\begin{array}{l}\text { CD/HSV-TK } \\
\text { HSV-TK }\end{array}$ \\
\hline & & NIS \\
\hline & & TRAIL \\
\hline (oncolytic) & Adenovirus & $\begin{array}{l}\text { OC promoter } \\
\text { PSA promoter }\end{array}$ \\
\hline \multirow[t]{25}{*}{ Immunotherapy } & AAV & GM-CSF \\
\hline & Adenovirus & IL-12 \\
\hline & & INF- $\beta$ \\
\hline & & MUC-1/CD40L \\
\hline & & p501 \\
\hline & & PSA \\
\hline & & PSMA \\
\hline & & PTVP-1 \\
\hline & Liposome & hTERT/lg heavy chain \\
\hline & & $\mathrm{IL}-2$ \\
\hline & & Prostatic acid phosphatase (PAP) \\
\hline & & PSA \\
\hline & & PSMA \\
\hline & Retrovirus & $\alpha(1,3)$ galactosyltransferase \\
\hline & & GM-CSF \\
\hline & & HPV-E6 \\
\hline & RNA & hTERT \\
\hline & & PSA \\
\hline & & Tumor RNA \\
\hline & Vaccinia & $5 \mathrm{~T} 4$ \\
\hline & & MUC-1/IL-2 \\
\hline & & PSA \\
\hline & & PSA/B7.1 \\
\hline & & PSA/B7.1/ICAM-1/LFA-3 \\
\hline & & PSA/B7.1/ICAM-1/LFA-3/GM-CSF \\
\hline
\end{tabular}


the cell phenotype. Nevertheless, in vivo correction of single gene defects have shown success in several preclinical and clinical studies.

p53. Tumor suppressor p53 is referred to as the molecular gatekeeper, protecting the integrity of the genome (144). When cellular DNA damage occurs, wild type p53 is activated and stimulates the expression of GADD45 (growth arrest and DNA damage-induced gene) and the cyclin-dependent kinase (CDK) inhibitor, p21. p21 inhibits the CDK-cyclin $\mathrm{D}$ complex required to phosphorylate $\mathrm{Rb}$, thereby halting the cell at the $G_{1} / S$ checkpoint to allow for DNA repair. If GADD45-mediated DNA repair is unsuccessful, p53 activates bax which mediates apoptosis (145). p53 mutations occur in approximately one third of early prostate cancers (146), and this increases in patients with advanced and metastatic disease (147). Replacement of wild-type p53 with recombinant adenoviral vectors (Ad-p53) resulted in growth inhibition and induction of apoptosis in prostate cancer both in vitro $(148,149)$ and in vivo $(149,150)$. In addition, intratumoral administration of Ad-p53 has been shown to slow the progression of localized prostate cancer to metastatic disease (151). Hernandez et al. described a natural variant of $\mathrm{p} 53, \mathrm{p} 53(\mathrm{R} 172 \mathrm{~L})$, in which an arginine-to-leucine mutation at codon 172 confered stronger protection against malignancy. When introduced into a transgenic adenocarcinoma of the mouse prostate (TRAMP) model, higher levels of bax were detected in addition to a lower incidence and reduced rate of prostate cancer growth (152). Perhaps the most powerful use of $p 53$ replacement is in combination with conventional therapies. Ad-p53 has been shown to sensitize prostate cancer cells in vitro and in vivo to DNA-damaging drugs such as cisplatin, doxorubicin, 5-fluorouracil (5FU), methotrexate and etoposide (153). Similarly, correction of p53 mutations with adenoviral vectors sensitized radio-resistant prostate cancer cells to radiation in vitro $(154,155)$. Cowen et al. described an additive effect when orthotopic LNCaP mouse 
xenografts were treated with both Ad-p53 and radiation therapy (156). Phase I/II clinical trials are ongoing to determine the safety of such therapies $(157,158)$.

Murine double minute clone 2. A key regulator of p53 is the oncoprotein murine double minute clone 2 (mdm2). It binds to the native tetrameric form of $p 53$ to inactivate its transcriptional function (159) or to promote the ubiquitination (160) and degradation (161) of p53. Mdm2 primarily binds to p53 in the N-terminal transactivation domain, overlapping the p300 and TAF binding sites (162). Overexpression of $m d m 2$ in prostate cancer is associated with more aggressive tumors, increased cell proliferation, and larger tumor volumes (163). Zhang et al. developed second-generation antisense oligonucleotides against $m d m 2$ to target the degradation of $m d m 2$ mRNA. This led to decreased proliferation, increased apoptosis, and sensitization to paclitaxel in LNCaP, PC-3 and DU-145 cell lines in addition to chemosensitization and growth suppression in LNCAP and PC-3 xenografts (164). As expected, antisense mdm2 sensitized prostate cancer cell lines and xenografts to radiation therapy $(165,166)$. Interestingly, the delivery of antisense $m d m 2$ to $\mathrm{LNCaP}$ cells restored the apoptotic response to androgen deprivation (167). The addition of antisense $m d m 2$ to existing p53 corrective gene therapy may enhance the antitumor effect and further sensitize prostate cancer to conventional therapies.

Phosphatase and tensin homologue. PTEN is a tumor suppressor with plasma membrane lipid phosphatase activity. Its primary function is to remove the 3 ' phosphate on phosphatidylinositol $(3,4,5)$-triphosphate (PIP3) in the phosphatidylinositol signaling cascade. Upon growth factor stimulation, phosphoinositide 3-kinase (PI3K) phosphorylates phosphatidylinositol $(3,4)$-bisphosphate (PIP2), generating PIP3 which transmits growth and survival signals. By regenerating PIP2, PTEN reverses the PI3K 
signal (168). Loss of PTEN occurs in $20 \%$ of prostate tumors and is associated with higher Gleason score and advanced stage cancer (31). PTEN loss also leads to altered cell cycle regulation (169), increased cellular invasion (170), enhanced cell survival (171), and stimulation of angiogenesis (172). Davies et al. demonstrated the inhibition of cell cycle progression in PC-3 cells infected with Ad-PTEN. Intratumoral injection of AdPTEN into orthotopic PC-3 tumors did not result in tumor growth inhibition; however, it suppressed metastasis formation (173). In Bcl-2 overexpressing PC-3 and LNCaP prostate cancer cell lines, Ad-PTEN sensitized cells to radiation therapy and induced a $\mathrm{G}_{2} / \mathrm{M}$ cell cycle arrest (174). Similarly, Ad-PTEN sensitized PC-3 and DU-145 cells to doxorubicin treatment (175).

\section{Oncolytic Viral Therapy}

Safety concerns of nonspecific viral replication in immune-compromised cancer patients limited the early clinical trials to the use of replication-deficient adenoviral vectors; however, previous studies have shown that the replication of adenovirus within a cell is sufficient to kill prostate cancer cells (176). By placing adenoviral early genes under the control of prostate-specific promoters, viral replication can be limited to prostate cells. This strategy allows the viral vector to propagate from a limited number of infected cells to the whole tumor mass, overcoming the problem of inadequate in vivo infectivity. This local viral amplification limits the number of injections required.

ONYX-015. The first tumor-specific oncolytic adenovirus, named ONYX-015, was developed by Bischoff et al. to replicate specifically in p53-deficient cells, a mutation common to several cancers. Tumor-specificity was achieved by deleting the adenoviral E1b-55K gene, whose gene product binds to and inactivates p53, enabling infected cells to enter S-phase and promote viral replication. Theoretically, normal cells would not be 
permissive to ONYX-015 replication. Originally, this virus was reported to demonstrate mutant p53-dependent replication (177), effective tumor regression following intratumoral or systemic injection in several nude mouse xenograft models including colon, cervical and laryngeal carcinomas $(178,179)$, and augmentation of tumor killing with co-administration of chemotherapeutic agents such as cisplatin and 5-fluorouracil (178). To date, ONYX-015 has been tested in sixteen phase I and II clinical trials for multiple malignancies including head and neck carcinoma, pancreatic cancer, ovarian cancer, colorectal cancer, hepatobiliary cancer, gastric cancer and gliomas. In these trials, no dose-limiting toxicities were observed; however, only modest to no objective responses were achieved (180-186). Although the virus has been shown clinically not to destroy normal tissues (187), several groups have reported that ONYX-015 replication is independent of $p 53$ status (188-190). Recently, O'Shea et al. reported that the tumorspecific replication of ONYX-015 is due to differential late viral mRNA export in malignant cells rather than the cell's p53 status (191). Despite the conflicting reports, ONYX-015 appears to favor viral replication in malignant cells over normal cells. The clinical utility of ONYX-015 for prostate cancer has not been tested, but prostate cancer gene therapy vectors with similar E1b-55K deletions have been developed (192).

Calydon virus 706 and 787 . The first prostate-specific replication-competent adenovirus (PSRCA) was developed by Rodriguez et al. Calydon virus (CV706) was engineered by placing the adenoviral E1a gene under the control of the minimal PSA promoter and enhancer sequences. In vitro, E1a expression was limited to PSA-positive LNCaP cells. Furthermore, in vivo analysis demonstrated the powerful antitumor efficacy of the virus against LNCaP mouse xenografts (193). When combined with radiation therapy, a significant synergistic effect was demonstrated both in vitro and in vivo in LNCaP xenograft models (194). CV706 was the first PSRCA tested in a human 
gene therapy clinical trial. The virus was found to be safe, as the maximum tolerable dose was not reached, and a drop in serum PSA greater than $50 \%$ was demonstrated in patients treated with the highest doses of CV706 (194). Yu et al. developed CV787, a PSRCA with higher killing efficiency than CV706. This virus retains the entire adenoviral E3 region, which enhances the virus' oncolytic effect. In addition, two promoters drive the expression adenoviral early genes. E1a is under the control of the prostate-specific rat probasin promoter, and $E 1 b$ is controlled by the human PSA promoter/enhancer. This virus replicated as efficiently as wild-type adenovirus and eliminated LNCaP xenograft tumors via tail vein injection (195). Further in vivo analysis revealed a synergistic enhancement of CV787 with both radiation therapy (196) and chemotherapeutic agents such as paclitaxel or docetaxel (197). Results from ongoing CV787 clinical studies are pending. Although CV706 and CV787 promise success in combination with conventional therapies, the androgen-dependency of the promoter systems used may limit their success.

Ad-OC-E1a and Ad-hOC-E1. Matsubara et al. developed a PSRCA using the mouse $O C$ promoter to restrict the expression of E1a to prostate epithelia and its supporting bone stroma in osseous metastases of prostate cancer. This virus, named Ad-OC-E1a, appears to be more effective than a PSA-controlled virus at killing a broader spectrum of prostate cancer cells including LNCaP, C4-2, and ARCaP (PSA-positive) as well as PC-3 and DU-145 (PSA-negative). Intratumoral injection of Ad-OC-E1a was effective at obliterating subcutaneous androgen-independent PC-3 athymic mouse xenograft models. In addition, intraosseous C4-2 prostate cancer xenografts responded very well to the systemic administration of Ad-OC-E1a. $100 \%$ of the treated mice responded with a drop in serum PSA below detectable levels. At the conclusion of the study, $40 \%$ of the treated mice were cured of prostate cancer, as no PSA rebound or 
prostate cancer cells in the skeleton were detected (198). To improve upon this virus, Hsieh et al. developed a second PSRCA, Ad-hOC-E1, containing a single bidirectional human $O C$ promoter to control the expression of both E1a and E1b (199). Previous studies have shown that controlling the expression of the early gene $E 1 b$ in addition to E1a results in better viral replication control (195). Under the control of this VDREcontaining promoter, Ad-hOC-E1 replication was induced 10-fold higher than wild-type viral replication and cytotoxicity was enhanced by the administration of vitamin D (199). Although still controversial (200), some preclinical studies indicate that vitamin D has an anti-proliferative effect on androgen-independent prostate cancer $(201,202)$. In preclinical studies, administration of vitamin $D_{3}$ in nude mice with subcutaneous DU-145 xenografts demonstrated a therapeutic effect; however, the systemic administration of Ad-hOC-E1 in combination with vitamin D showed marked repression of the tumors, indicating the potential for clinical use (199).

Ad-E4PSESE1a. As discussed above, the control of viral replication has been achieved by tightly controlling both $E 1 a$ and $E 1 b$ adenoviral genes under prostatespecific promoters. Recent studies have also demonstrated that tight control of viral replication can be achieved by placing E4 in addition to E1a under the control of two separate or duplicate tissue-specific promoters $(203,204)$. Due to the difficulty of finding two active and tightly regulated promoters for the prostate and because promoter duplication might induce recombination, $\mathrm{Li}$ et al. developed a novel strategy to control E1a and E4 genes under a single promoter. This is also the first report of the use of PSES to drive the replication of a PSRCA. The virus, Ad-E4PSESE1a, also contains the gene encoding green fluorescent protein (GFP) driven by CMV promoter for the purpose of in vivo viral tracking. In vitro, the replication and cell killing abilities of this virus were similar to that of wild-type adenovirus in PSA/PSMA-positive cells. As expected, 
replication of Ad-E4PSESE1a was severely impaired in PSA/PSMA-negative cells. AdE4PSESE1a effectively suppressed the growth of androgen-independent CWR22rv mouse xenograft tumors (205). The oncolytic properties of this virus could be augmented by the replacement of CMV-GFP with a therapeutic gene under control of a prostate-specific promoter.

Overcoming current limitations of oncolytic vectors. Through the use of intravital imaging, Li et al. mapped the replication and spread of a PSRCA throughout prostate xenografts. Three days after Ad-E4PSESE1a injection, a burst in GFP expression was observed, indicating rapid replication and viral spread, which decreased one week after injection. At two weeks, tumor growth and cell killing reached equilibrium. After this, tumor growth exceeded the killing rate. A subsequent rebound in GFP expression was observed, indicating renewed viral amplification. Although immunohistochemistry analysis revealed persistent viral infection for up to 28 days, the xenograft tumors did not completely respond to the viral therapy (205). Other studies have also described limited viral spread and lack of tumor response despite the high oncolytic activity of a virus (206). One explanation for this is that conditions within the established tumor may become unfavorable to support viral replication. As tumor lysis occurs, the tumor environment becomes highly necrotic and hypoxic, unlike the welloxygenated tissues adenovirus usually infects. The fact that virus particles accumulate around blood vessels within a tumor provides further supporting evidence (207). Shen et al. found that levels of E1a protein but not mRNA are reduced in Ad-infected cells under hypoxic conditions (208). The significance of this finding is great because transcription of adenoviral genes, and therefore adenoviral replication, is dependent on E1a. Further work in this area is needed to improve the stability of E1a under hypoxic conditions. 
A second hypothesis to explain the poor tumor response to oncolytic therapy also involves the hypoxic environment created within the tumor. Such a condition would induce angiogenesis, and the resulting neovasculature could then support new tumor growth. Eventually, this growth may overcome viral replication. If this is a significant factor in lowering the efficacy of oncolytic viral therapy, then the use of an antiangiogenic factor as adjuvant therapy should enhance the tumor response to oncolytic therapy. Recently, Li et al. combined the PSRCA Ad-E4PSESE1a with a replication-defective adenovirus expressing the anti-angiogenic fusion product of endostatin and angiostatin, called EndoAngio. It has been reported that replication-deficient vectors can co-amplify along with replication-competent vectors, resulting in enhanced transgene expression (209). When co-administered in CWR22rv androgen-independent prostate xenografts in nude mice, 7 of 8 tumors completely regressed, while one demonstrated growth suppression for at least 14 weeks post-treatment (210). Because the co-administration of multiple adenoviral vectors could result in reduced gene transduction efficiency, due to the need for both vectors to infect each tumor cell, Li et al. improved this therapy by inserting the EndoAngio expression cassette into a PSRCA. The results were more dramatic, with 9 out 10 CWR22rv xenografts demonstrating complete regression and one tumor remaining dormant for at least 28 weeks (211).

Another limitation to the use of early tissue-restricted replicative adenoviruses was the inability to deliver large or multiple therapeutic transgenes. Critical vector backbone modifications have been made to allow for larger insert sizes. The first modification is the chimeric Ad5/35 vector which retargets the virus by substituting the fiber from Ad5, which binds to CAR, with the shorter fiber from Ad35, which uses CD46 as its cellular receptor. The resulting chimeric genome is 756 bp shorter than wild-type Ad5. In addition to this packaging advantage, Ad5/35 has greater infectivity for prostate cancer cells than Ad5, and neutralizing antibodies to $\mathrm{Ad} 35$ are not as prevalent as Ad5 
neutralizing antibodies (81). Modification of the adenoviral E4 region also results in genomic space conservation. Open reading frames 1,2 and 4 encode proteins with minimal or unknown function on viral replication and the lytic process (212), and the function of the gene products of open reading frames 3 and 6 overlap (213). Deletion of open reading frames 1 to 4 does not significantly alter viral replication in tumor cells (213), and the deletion results in approximately $1.2 \mathrm{~kb}$ greater insert size. The E3 region encodes several proteins that enable an infected cell to evade the host immune system and prevent apoptosis induction, functions that are not critical for viral replication (66). In addition, it encodes ADP which is expressed late in infection and necessary for efficient cell lysis (72). By removing the E3 region and reinserting the ADP coding sequence, approximately $2 \mathrm{~kb}$ of genomic space is conserved. If all three vector backbone modifications are performed, approximately $4 \mathrm{~kb}$ additional space is allowed for insertion of therapeutic transgenes and transcriptional control elements.

One final consideration is the circumvention of the host's immune response. Transgene expression is limited by the adaptive cellular responses mounted against transduced cells. One possible solution is to temporarily suppress the host's immune system; however, shut-down of the entire immune system could be problematic during infection with replication-competent adenoviral vectors. This has been attempted using cyclosporin A (214) and antibodies to deplete both CTLs and CD4 T-cells $(215,216)$. Activation of innate immunity also limits viral transduction efficiency and limits availability of active viral particles. Previous studies have attempted to deplete macrophages with limited clinical utility (217). Perhaps more appealing than systemic immunosuppression would be the local delivery of an immunomodulator. This could best be achieved by direct delivery via the oncolytic vector. Among the immune regulators, TGF- $\beta$ is likely to the best candidates for incorporation into a PSRCA. TGF- $\beta$ is a mediator of immune suppression that allows tumors to escape immune surveillance (218), and its expression 
in transduced tumor cells would decrease activation of macrophages, natural killer cells and CTLs. Although local suppression of innate and adaptive immunity would be ideal to enhance transgene expression, it would inhibit any potential anti-tumor immune responses. A third approach is to suppress the humoral anti-adenoviral immunity. As stated previously, modifications to the capsid, including the fiber knob can decrease humoral neutralization of the vector. Covalent modification of the viral capsid with inert compounds such as polyethylene glycol (PEG) has been described as a means to mask the neutralizing epitopes on adenoviral vectors (219). Importantly, this modification is performed after viral production and purification, does not inhibit cell penetration and infectivity (219), and reduces both innate (220) and adaptive immune responses (221); however, its benefit would only be realized for one viral replication cycle.

\section{Cytotoxic Gene Therapy}

This approach to the molecular therapy of prostate cancer results in the killing of cancer cells by delivery and expression of pro-drug enzyme genes such as herpes simplex virus thymidine kinase (HSV-TK) or cytosine deaminase (CD) and apoptosisinducing genes such TRAIL or FasL. To ensure the safety of such a therapy, the use of prostate-specific promoters is crucial to protect non-target tissues from the expression of such deadly gene products. Furthermore, the use of PSRCA vectors to deliver the cytotoxic genes overcomes the need for multiple doses of the virus due to limited viral transduction and poor transgene expression.

Herpes simplex virus-thymidine kinase. In this approach HSV-TK is delivered to prostate cancer cells followed by systemic administration of any one of several antiherpetic agents such as acyclovir (ACV), ganciclovir (GCV) or valacyclovir (VAL). These nucleoside analogues are phosphorylated specifically by HSV-TK, allowing incorporation 
of the activated analogues into cellular DNA during DNA replication, resulting in chain termination and ultimately cell death. The safety of this therapy resides in the fact that only the cells receiving HSV-TK are capable of converting the non-toxic pro-drug into an active metabolite. Fortunately however, the toxic effect is not limited to the cells in which the pro-drug is activated. Through a bystander effect mediated by intercellular gap junctions and phagocytosis of debris from dying cells, the activated drug is delivered to neighboring cells (222), resulting in an amplification of the cytotoxic effect throughout the entire tumor site.

Previously, Eastham et al. demonstrated the sensitivity of human prostate cancer cells PC-3 and DU-145 to GCV cytotoxicity following the in vitro transduction of the cells with HSV-TK using a recombinant replication-deficient adenoviral vector (223). Similar results were obtained in vivo in murine subcutaneous xenograft prostate cancer models following the intralesional injection of Ad-RSV-TK and Ad-CMV-TK (224, 225). Intratumoral injection of the vector is required if universal promoters such as RSV or CMV are used to drive the expression of HSV-TK. Herman et al. developed the initial HSV-TK clinical trial in which a replication-deficient adenovirus carrying HSV-TK driven by RSV promoter was injected intralesionally, followed by administration of GCV in men with locally recurrent prostate cancer one or more years after definitive external beam radiotherapy. This trial demonstrated the tumoricidal activity of this combination therapy, as evidenced by sustained decreases in serum PSA. Unfortunately, several of the patients experienced self-limiting toxicities and one patient experienced moderate but reversible hepatic dysfunction and thrombocytopenia (99). To circumvent such toxicities, Gotoh et al. developed a replication-deficient adenoviral vector which controlled HSV-TK expression by the PSA promoter (106).

The first transcriptionally-targeted HSV-TK gene therapy to be translated into a clinical protocol was developed by Koeneman et al. to test the hypothesis that the OC 
promoter could regulate HSV-TK expression specifically within prostate cancer cells and the supportive stroma of a metastasis (134). Kubo et al. performed the phase I clinical trial in which two post-surgical local recurrences and nine metastatic lesions (five osseous and four lymph nodes) were injected with replication-defective Ad-OC-TK vector followed by the administration of oral VAL. All patients tolerated this therapy with no severe adverse effects. Of the eleven men, local cancer cell death was observed in seven patients; however, the treated lesions of all eleven men showed histological changes as a result of the treatment. One patient demonstrated regression and stabilization of the treated lesion for up to 317 days post-treatment without additional treatments (135). In follow-up Phase I/II testing of this virus, one of three men treated with intralesional injection of Ad-OC-TK demonstrated maintenance of a drastically reduced serum PSA for over 200 days post-therapy and complete radiographic regression of a treated lumbar metastasis by 180 days post-treatment (226). To enhance transcriptional activity and transduction efficiency, Ahn et al. incorporated a PSES-HSV-TK expression cassette into a PSRCA called Ad-IU1 (227).

To improve the efficacy of HSV-TK gene therapy, Freytag et al. developed a novel three-legged approach to gene therapy for prostate cancer in which HSV-TK was fused with $C D$ cDNA and delivered within an oncolytic virus, followed by treatment with external beam radiation therapy (192). In this approach, CD, an enzyme found only in bacteria and certain yeast, converts the pro-drug 5 -fluorocytosine $(5-\mathrm{FC})$ to a highly toxic nucleoside analogue, 5-FU, conferring an additive effect to HSV-TK gene therapy. This virus, Ad5-CD/TKrep, is based on the oncolytic ONYX-015 viral backbone. In the initial phase I study, this virus showed moderate efficacy as demonstrated by decreased serum PSA values for several patients and complete tumor destruction in two of sixteen patients one year post-treatment (228). In murine orthotopic C4-2 tumors, Ad5$\mathrm{CD} / \mathrm{TK}$ rep was shown to be an effective adjuvant to radiation therapy (229). This 
efficacy was tested in a phase I study which combined Ad5-CD/TKrep prodrug therapy with radiation therapy. All patients experienced a decrease in serum PSA, with a mean PSA half-life of 0.6 months, versus two months for patients receiving Ad5-CD/TKrep without radiation therapy or 2.4 months for patients receiving radiation therapy alone. Furthermore, up to nine months post-treatment, five out of ten patients maintained PSA levels $\leq 0.5 \mathrm{ng} / \mathrm{ml}(230)$.

\section{Immunotherapy}

Prostate cancer, like most cancers, has developed mechanisms to evade the host immune system. Such mechanisms include the down-regulation of class I major histocompatibility complex (MHC) molecules on the tumor cell surface (231) as well as the down-regulation of the co-stimulatory B7 molecules (232). These means of evasion result in decreased presentation of tumor antigens to CD-4 T lymphocytes. The goal of immunotherapy is to enhance the host immune response to prostate cancer cells. Current approaches involve ex vivo gene therapy of autologous or allogeneic tumor cells and subsequent vaccination with the irradiated cells now expressing cytokines such as interleukin 2 (IL-2) and granulocyte-macrophage colony-stimulating factor (GM-CSF), ex vivo gene transfer of prostate-specific cDNAs, such as PSA, PSMA and prostatic acid phosphatase (PAP), into autologous dendritic cells (DCs), and in vivo intratumoral gene transfer of cytokine genes.

Prostate Cancer Vaccines. The host immune system is capable of recognizing and eliminating malignant cells; however, the ability of tumor cells to evade immune surveillance and the inefficiency of the body's antitumor response allows prostate cancer to persist and progress. To develop a tumor vaccine, prostate cancer cells are harvested from the patient during radical prostatectomy, transfected with cytokines that 
upregulate cell surface antigens such as B7 or MHC class I and II, expanded in culture, and irradiated to kill the cells. After infusion of the autologous vaccine cells, the enhanced immunogenicity of the cells aids the immune system to mount a local or systemic immune response to the cancer $(233,234)$. The first prostate vaccine clinical trial was developed by Simons et al. In this study, eight men with metastatic prostate cancer were administered autologous, GM-CSF-transduced, irradiated cancer vaccines. Side effects were limited to pruritus, erythema and swelling at the site of vaccination. Both $B$ and $T$ cell responses were observed in seven of the eight patients, while $T$ cell responses were observed in only two of the eight prior to vaccination (235). A subsequent clinical trial demonstrated no therapeutic value of systemic administration of recombinant GM-CSF, thereby suggesting the importance of local secretion of GM-CSF by the cancer vaccines (236). Clearly, a limitation of this approach is the harvesting and ex vivo manipulation of prostate cancer cells. To overcome this, Simons et al. developed an allogeneic tumor vaccine, GVAX, from GM-CSF-transduced irradiated PC3 and LNCaP prostate cancer cell lines. In a phase II clinical trial, the vaccines were well tolerated, and no dose-limiting toxicities were observed. Two years post-treatment, the survival rate of patients receiving low booster doses was $41 \%$, compared to $70 \%$ in patients who received higher booster doses (237). A phase I/II clinical trial evaluated the safety of GVAX reengineered to secrete higher levels of GM-CSF (238). Phase III clinical studies of this cancer vaccine are underway.

Dendritic Cell Immunotherapy. A second approach to prostate cancer vaccination involves the use of dendritic cells (DCs), the most potent antigen presenting cell (APC) of the immune system. DCs produce a strong systemic T cell response by presenting tumor antigens on both MHC class I and II molecules (239). In this vaccination strategy, autologous DCs are collected from the patient by leukopheresis, 
expanded in culture, challenged with a prostate-specific antigen, such as PSA, PAP or PSMA by either peptide pulsing or transfection with antigen CDNA, and reintroduced into the patient. Murphy et al. demonstrated the safety of HLA-A2-specific PSMA peptidepulsed DC immunotherapy in a phase I clinical trial in which an enhanced cellular immune response was observed in all HLA-A2-positive patients; however, only seven out of fifty-one patients experienced a decrease in serum PSA $\geq 50 \%(240,241)$. In a phase II clinical trial, GM-CSF was administered as a systemic adjuvant in patients receiving PSMA-pulsed autologous DC vaccines. Nineteen out of sixty-two patients were identified as partial or complete responders. Of the responders, $58 \%$ appeared to have durable responses $(236,242)$.

In Vivo Immunotherapy. A third approach to immunotherapy for prostate cancer is the delivery of cytokines to the local tumor environment. IL-2 is a potent activator of CD4+ and CD8+ T cells as well as natural killer (NK) cells, and its effect on prostate cancer is due to its ability to expand T cell populations with antitumor activity. Tumors that respond to IL-2 therapy demonstrate an infiltration of lymphocytes. In mouse PC-3 orthotopic tumor models, tumor growth was suppressed by $94 \%$ following systemic injection of IL-2 (243). Belldegrun et al. developed a gene therapy protocol in which twenty-four men with locally advanced prostate cancer were injected intraprostatically with a DNA-liposome complex encoding the IL-2 gene. This therapy was well tolerated. Immunohistochemical analysis of the tumor site demonstrated T cell infiltration, and serum PSA responses were observed in sixteen of the men on day one and fourteen of the twenty-four on day eight (244). Trudel et al. developed a phase I clinical trial to evaluate the safety of intraprostatic injection of adenoviral vectors encoding the IL-2 gene. Twelve men were injected with Ad-IL-2 four weeks prior to prostatectomy. No dose-limiting toxicities were observed. On pathological evaluation, 
all patients experienced a local inflammatory response with an infiltrate of CD3+CD8+ cells. At low viral doses, five out of five patients experienced a mean decrease in PSA of $33 \%$, while patients receiving the highest viral doses experienced transient elevations in PSA levels before returning to baseline (245). IL-12 is also under investigation as a potent immunotherapy for prostate cancer. In addition to activation of NK cells and CTLs, IL-12 has direct cytotoxic effects on tumor cells and anti-angiogenic properties (246, 247).

\section{Thesis Overview}

Dose-limiting toxicities and treatment resistance with conventional therapies for advanced prostate cancer warrant the development of novel therapeutic strategies. The studies described in this thesis characterize a novel molecular therapy for advanced prostate cancer and examine its efficacy in combination with conventional therapy. The first aim of the thesis is to determine whether the addition of the cytotoxic transgene TRAIL will enhance the in vitro and in vivo anti-tumor activity of a replication-competent adenovirus against androgen-independent prostate cancer. The second aim is to determine whether the neoadjuvant treatment of advanced prostate cancer with a PSCRA expressing TRAIL will result in the radio-sensitization of tumor cells, thereby enhancing the therapeutic effect of EBRT. The third aim is to delineate the mechanism responsible for the enhancement of the therapeutic efficacy of EBRT by a PSRCA expressing TRAIL. I expect that these studies will provide insight into new treatment modalities for high risk prostate cancer and lead to translation to a clinical trial. 


\section{Materials and Methods}

\section{Cell Culture}

The packaging cell line HER911E4 stably expresses the adenoviral E4 gene under control of the inducible tetR promoter (248) and was derived from the human embryonic retinoblast (HER911) cell line which was transformed with a plasmid containing the adenoviral genome (bp 79-5789) (249). HER911E4 cells were cultured in DMEM supplemented with 10\% FBS (Atlanta Biologicals, Lawrenceville, GA), 1\% penicillin-streptomycin (Gibco, Grand Island, NY), $0.1 \mathrm{mg} / \mathrm{ml}$ hygromycin B (Calbiochem, San Diego, CA) and $2 \mu \mathrm{g} / \mathrm{ml}$ doxycycline (Sigma, St. Louis, MO). To induce adenoviral E4 gene expression, HER911E4 cells were cultured in medium without doxycycline for 24 hours prior to infection. CWR22rv is an androgen-independent, PSA/PSMA-positive prostate cancer cell line derived by the propagation of the androgen-dependent parental xenograft, CWR22, in nude mice (250). LNCaP is an androgen-dependent, PSA/PSMApositive prostate cancer cell line established from a lymph node of a patient with metastatic disease (251). C4-2, an androgen-independent, PSA/PSMA-positive prostate cancer line, was derived by co-injection of $\mathrm{LNCaP}$ and bone stromal cells into nude mice (252). PC-3 is an androgen-independent, PSA/PSMA-negative prostate cancer cell line that was originally derived from the bone marrow aspirates of a patient with bone metastases (253). DU-145, an androgen-independent prostate cancer cell, is PSA/PSMA-negative and was derived from a brain lesion from a patient with confirmed metastatic disease (254). Prostate cancer cell lines used in these studies (Table 3) were cultured in RPMI 1640 supplemented with 10\% FBS and 1\% penicillin and streptomycin. Adult human dermal fibroblasts (HDFa) were cultured in Medium 106 supplemented with $2 \%$ FBS, $1 \mu \mathrm{g} / \mathrm{ml}$ hydrocortisone, $10 \mathrm{ng} / \mathrm{ml}$ human epidermal growth factor, $3 \mathrm{ng} / \mathrm{ml}$ basic fibroblast growth factor and $10 \mu \mathrm{g} / \mathrm{ml}$ heparin (Cascade Biologics, Portland, OR). LoVo 
is a colorectal adenocarcinoma cell line derived from a supraclavicular lymph node of a patient with metastatic Dukes' type C, grade IV disease (255). T24 was established from a transitional cell carcinoma of the urinary bladder (256). LoVo and T24 cell lines were cultured in RPMI 1640 media supplemented with 10\% FBS and 1\% penicillin and streptomycin. All cells were maintained in a humidified incubator at $37^{\circ} \mathrm{C}$ and $5 \% \mathrm{CO}_{2}$.

Table 3. Characterization of prostate cancer cell lines used in these studies. PSES is transcriptionally active in PSA/PSMA-positive cells. Common mutations in prostate cancer cell lines include androgen receptor (AR) and tumor suppressor p53.

\begin{tabular}{ccccc}
\hline Cell Line & PSA/PSMA & Androgen & Mutations & Origin \\
\hline CWR22rv & + & Independent & AR, p53 & Primary tumor \\
LNCaP & + & Dependent & AR & Lymph node met. \\
C4-2 & + & Independent & AR & LNCaP derivative \\
PC-3 & - & Independent & p53 & Lumbar met. \\
DU-145 & - & Independent & p53 & Brain met. \\
\hline
\end{tabular}

\section{Production of Adenoviral Vectors}

Ad-IU2 was developed by modifying Ad-E4PSESE1a, the previously described PSRCA with a CMV promoter-driven enhanced green fluorescent protein (EGFP) marker (205). To construct Ad-IU2, human full-length TRAIL cDNA from pORF-hTRAIL (InvivoGen, San Diego, CA) was cloned downstream of PSES into pAd1020SfidA (OD 260, Boise, ID), the adenoviral cloning vector containing the left ITR and packaging signal, to make pAd1020SfidA-PSES-TRAIL, which was further digested with Sfil to release the left ITR and PSES-TRAIL expression cassette. This fragment was cloned into pAd288E1b-E4PSESE1a (205), the modified adenoviral genome vector, and the ligation product was transformed into TOP10 E. coli competent cells (Invitrogen, 
Carlsbad, CA). The adenoviral genome was released by digestion with $\mathrm{Pacl}$ and transfected into HER911E4 cells with Lipofectamine 2000 (Invitrogen) at 80\% confluency in a 6-well plate. After 7 to 10 days, once cytopathic effect of the virus was evident by light microscopy, the viral supernatant was harvested by 3 rounds of freezing (mixture of 95\% ethanol and dry ice) and thawing $\left(37^{\circ} \mathrm{C}\right.$ water bath). To further amplify Ad-IU2, HER911E4 cells were infected with the viral supernatants, in succession from a P60 dish, to a T75 flask, to a triple flask, and finally to 10 triple flasks. The final viral supernatant was applied to a CsCl gradient $(1.5 \mathrm{~g} / \mathrm{ml}, 1.35 \mathrm{~g} / \mathrm{ml}$ and $1.25 \mathrm{~g} / \mathrm{ml})$ and centrifuged at $75,000 \times \mathrm{g}$ and $10^{\circ} \mathrm{C}$ for 1 hour. The adenoviral band, found between the $1.25 \mathrm{~g} / \mathrm{ml}$ and $1.35 \mathrm{~g} / \mathrm{ml}$ gradients, was applied to $1.35 \mathrm{~g} / \mathrm{ml} \mathrm{CsCl}$ and centrifuged overnight at $75,000 \times \mathrm{g}$ and $10^{\circ} \mathrm{C}$. The double gradient-purified adenoviral stock was dialyzed for 24 hours against a dialysis buffer of $1 \mu \mathrm{M} \mathrm{MgCl}_{2}, 10 \mu \mathrm{M}$ Tris- $\mathrm{HCl}(\mathrm{pH} 7.5)$ and $10 \%$ glycerol, changing the buffer every 8 hours. Dialyzed virus was aliquoted and stored at $-70^{\circ} \mathrm{C}$. Experiments were repeated using a second batch of adenovirus to verify results.

Replication-competent control viruses used in this study include Ad-E4PSESE1a and Ad-IU1. Ad-IU1 was constructed in a similar fashion as Ad-IU2; however, a PSESHSV-TK expression cassette replaces the PSES-TRAIL expression cassette (227). Without administration of a nucleoside analog prodrug, the only cytotoxicity provided by Ad-IU1 is due to replication. As a replication-defective control, Ad- $\triangle T A T A-E 1 a$, in which the E1a TATA box was deleted from the Ad-E4PSESE1a viral backbone, was used. To achieve equal bioactivity of Ad-IU2 and control viruses, a titer assay was performed. $1 \mathrm{x}$ $10^{4}$ HER911E4 cells were plated overnight in 96-well plates and infected with serial dilutions of Ad-IU2, Ad-IU1, Ad-E4PSESE1a or Ad- $\triangle$ TATA-E1a, so that 10 wells in every row received the same dose, ranging from $10^{-3}$ to $10^{-10}$. Media were changed 24 hours after infection, and cells were observed daily under light microscopy for cytopathic effect. 
7 days after infection, lethal dose $\left(\mathrm{LD}_{50}\right)$ was determined to be the dose at which $50 \%$ of cells or greater were killed (at least 5 wells per row). Viral titers were calculated as $L_{50}$ units (LDU) per $\mu \mathrm{l}$. Conversions from viral particles (vp) to LDU were as follows: Ad-

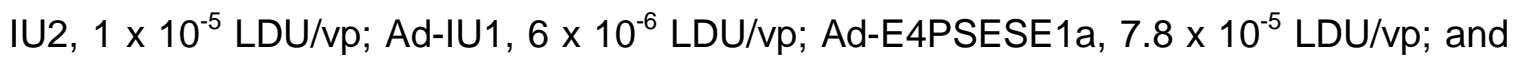
Ad- $\triangle$ TATA-E1a, $1.5 \times 10^{-5}$ LDU/vp.

\section{Western Blot Analysis}

For TRAIL expression, 1 × $10^{6}$ CWR22rv cells were cultured overnight in 6-well plates and infected with $0.01 \mathrm{LDU} /$ cell Ad-IU2 or Ad- $\triangle T$ TATA-E1a. As a positive control, CWR22rv cells were transfected with pORF-hTRAIL using Lipofectamine 2000. Media were changed 24 or 3 hours after infection or transfection, respectively. For adenoviral E1a expression, $1 \times 10^{6}$ C4-2, CWR22rv, DU-145 and PC-3 cells were cultured overnight in 6-well plates and treated with Ad-IU1, Ad-IU2, Ad5-Wt and PBS at 100 vp/cell for C4-2 and CWR22rv and 1000 vp/cell for DU-145 and PC3 cells. Media were changed 24 hours after infection. 48 hours after infection, cells were washed with cold PBS and harvested with radioimmunoprecipitation assay (RIPA) buffer containing $1 \mathrm{ml}$ modified RIPA buffer, $20 \mu \mathrm{l} 57 \mathrm{mmol} / \mathrm{L}$ phenylmethylsulfonyl fluoride and $2.5 \mu \mathrm{l}$ phosphatase inhibitor cocktail (Sigma-Aldrich, St. Louis, MO). Cell lysates were kept on ice for 1 hour, centrifuged to pellet debris, and supernatants kept at $-70^{\circ} \mathrm{C}$. To determine whether cleaved TRAIL was present in Ad-IU2 conditioned media, $1 \times 10^{6}$ CWR22rv cells were infected with $0.01 \mathrm{LDU} /$ cell, as above. 48 hours after infection, the medium was harvested, centrifuged at $800 \times \mathrm{g}$ for 10 minutes, and the cell lysate was prepared as above. As positive controls, various dilutions from 100 to $25 \mathrm{ng} / \mathrm{ml}$ recombinant human TRAIL (rhTRAIL) (BioSource, Camarillo, CA) were loaded. Protein concentration was analyzed by Bradford assay (Bio-Rad, Hercules, CA), and $20 \mu \mathrm{g}$ of protein (with 0.3 M DTT) were separated by 10 or $12 \%$ SDS-PAGE and transferred to a nitrocellulose 
membrane. Membranes were blocked overnight at $4^{\circ} \mathrm{C}$ in $5 \%$ fat-free milk and TBST and incubated with primary antibodies against human TRAIL (Santa Cruz Biotechnology, Santa Cruz, CA), $\beta$-actin (Sigma) or Ad5 E1a (BD Biosciences, Franklin Lakes, NJ). Blots were incubated with appropriate HRP-conjugated secondary antibodies, and specific binding was detected by ECL (Pierce, Rockford, IL).

\section{Measurement of TRAIL Surface Expression}

$1 \times 10^{5}$ CWR22rv cells were plated overnight in 24-well plates and infected with $0.01 \mathrm{LDU} /$ cell Ad-IU2 or Ad-IU1 or treated with PBS. Fresh media were replaced 24 hours after infection. 48 hours post-infection, cells were harvested and resuspended in $100 \mu \mathrm{l}$ ice cold staining buffer (PBS containing 10\% FBS and $1 \%$ sodium azide) in fluorescence-activated cell sorting (FACS) tubes. Cells were stained with PEconjugated anti-human TRAIL antibody (Abcam, Cambridge, MA) for 1 hour at $4^{\circ} \mathrm{C}$, protected from light. Cells were washed twice with ice cold PBS and resuspended in $500 \mu \mathrm{l}$ staining buffer. Analysis was performed on a FacScan flow cytometer (BD Biosciences) and data analyzed using WinMDI 2.9 software.

\section{Viral Replication Assay}

$1 \times 10^{6}$ CWR22rv, C4-2, LNCaP, PC-3 and DU-145 prostate cancer cell lines were seeded in 6-well plates overnight and infected with standardized doses of virus based on each cell line's infectivity (205). Media were changed 24 hours after infection, and cells were observed daily by light microscopy for cytopathic effect. Viral supernatants were harvested 3 days after infection by subjecting the cells and media to three freeze-thaw cycles and centrifuging to remove the cell debris pellet. HER911E4 cells were plated in 96-well plates and infected with serial dilutions of viral supernatant ranging from 1 to $10^{-11}$, so that every well in each column received the same dose. Cells 
were examined for cytopathic effect on day 7 , and $L_{50}$ was recorded as the dose causing cytopathic effect in at least four of eight wells in one column.

\section{Measurement of Apoptosis Induction}

CWR22rv, C4-2, LNCaP, PC-3 and DU-145 prostate cancer cells were seeded in 24-well plates overnight and infected with $0.01 \mathrm{LDU} /$ cell Ad-IU2 or Ad-IU1 or treated with PBS. 24 hours after infection, media and cells were harvested, washed with PBS and resuspended in $100 \mu$ l binding buffer. LNCaP cells were also infected with $0.01 \mathrm{LDU} /$ cell Ad-IU2 or Ad-IU1 or treated with PBS, and 48 hours after treatment, media and cells were harvested, washed with PBS and resuspended in $100 \mu \mathrm{l}$ binding buffer. Cells were stained with $2.5 \mu \mathrm{l}$ each of Annexin V-FITC and propidium iodide (PI) (BD Biosciences, Pharmingen) for 15 minutes at room temperature and analyzed by FACS analysis as above. Cells that were single-positive for Annexin V-FITC or double-positive for Annexin V-FITC and PI were considered positive for apoptosis.

\section{In Vitro Cell Killing Assay}

CWR22rv, C4-2, LNCaP and HDFa cells were seeded in 24-well plates overnight and treated with various doses of Ad-IU2, Ad-E4PSESE1a, Ad- $\triangle T A T A-E 1 a$ or PBS. Media were replaced with fresh medium 24 hours after infection, and cells were maintained in culture, changing media every other day, until a cytopathic effect was observed under light microscopy. Once a cytopathic effect was evident, cells were fixed with $1 \%$ paraformaldehyde, washed twice with cold PBS, stained with $0.5 \%$ crystal violet solution for 10 minutes and washed with cold tap water. To quantitate the remaining attached cells, stained cells were permeabilized with $1 \%$ SDS and analyzed for optical density at $570 \mathrm{~nm}$ on a Spectra Max Plus spectrophotometer (Molecular Devices, Sunnyvale, CA). Relative cell number was determined as the ratio of the $A_{570}$ value for 
Ad-IU2-, Ad-E4PSESE1a- or Ad- $\triangle$ TATA-E1a-treated cells to the $A_{570}$ value for untreated (PBS) cells at each viral dose and was used to evaluate cell survival.

\section{Evaluation of Bystander Effect}

CWR22rv cells were plated in a 12-well plate overnight and infected with 0.01 LDU/cell Ad-IU2 or Ad-IU1 or treated with PBS. 24 hours post-infection, cells were washed three times with cold PBS to remove residual virus, and fresh medium was replaced. PC-3 cells stably expressing a fusion of humanized Renilla luciferase (hrl) and monomeric red fluorescent protein (mrfp) reporter genes were co-cultured with the CWR22rv cells at a ratio of 3 CWR22rv cells to 1 PC-3 cell. The plasmid conferring expression, pcDNA3.1-CMV-hrl-mRFP, was constructed from pcDNA3.1-CMV-hrl-mrfpttk (a gift from Dr. Sanjiv Gambhir, Stanford University, CA) by deleting truncated thymidine kinase (ttk) from the vector. 24 hours after co-culture, media and cells were harvested, washed with PBS and resuspended in $100 \mu$ l binding buffer. Cells were stained with $2.5 \mu \mathrm{l}$ Annexin V-FITC for 15 minutes at room temperature and analyzed by FACS analysis as above. Percent apoptotic PC-3 cells was determined as the fraction of Annexin V-FITC-positive cells in the mRFP-positive population.

To determine whether direct cell-to-cell contact was required to mediate a bystander effect, CWR22rv cells were seeded overnight in 6-well plates and infected with 0.01 LDU/cell Ad-IU2, Ad-IU1 or Ad-E4PSESE1a. 48 hours after infection, media were harvested, centrifuged at $800 \mathrm{~g}$ for 10 minutes to remove dead cells and debris, and heat-inactivated at $56^{\circ} \mathrm{C}$ for 30 minutes. CWR22rv or PC-3 cells were seeded in 24-well plates overnight and treated with the heat-inactivated medium for 24 hours, at a ratio of 1:1. Media and cells were harvested and analyzed for apoptosis by FACS analysis as described above. To determine whether adenovirus was inactivated by heat-treatment, CWR22rv cells were seeded onto 24-well plates overnight and treated with conditioned 
medium from Ad-E4PSESE1a-infected CWR22rv cells, as above, before or after heatinactivation at $56^{\circ} \mathrm{C}$ for 30 minutes. 24 hours after treatment, cells were washed with cold PBS, harvested, fixed with $2 \%$ paraformaldehyde, resuspended in PBS, and analyzed for GFP expression by FACS analysis.

\section{In Vivo Evaluation of Ad-IU2 Anti-Tumor Effect}

CWR22rv xenografts were established by injecting $2 \times 10^{6}$ CWR22rv cells subcutaneously (SQ) into the flanks of 6 week-old male athymic nude mice. One week after injection, mice were anesthetized and bilateral orchiectomies were performed to ablate the production of androgens. Once tumors were established (33.5 to $65.5 \mathrm{~mm}^{3}$ ), mice were injected intratumorally with $2 \times 10^{4}$ LDU Ad-IU2, Ad-IU1 (PSRCA control) or PBS (vehicle control). Tumor sizes were monitored weekly, and tumor volumes were calculated as $\left(\mathrm{L}^{2} \times \mathrm{W}\right) /(\pi / 6)$. Tumor data was presented as fold-increase in tumor size relative to initial size at time of treatment. Mice were sacrificed at 6 weeks, and tumors were harvested, fixed in formalin and embedded in paraffin. All animal procedures were approved by the Indiana University School of Medicine Institutional Animal Care and Use

Committee (IACUC). Tumor sections were deparaffinized with xylene, hydrated in ethanol and distilled water, and stained with Hematoxylin and Eosin (H\&E). Tumor sections were evaluated for in situ apoptosis using a fluorometric terminal dUTP nickend labeling (TUNEL) assay (Promega, Madison, WI). Nuclei were counterstained with DAPI and tumor sections visualized by confocal microscopy on a Bio-Rad MRC1024 laser scanning dual-photon confocal microscope (Bio-Rad).

\section{Clonogenic Assay}

$1 \times 10^{6}$ CWR22rv or C4-2 cells were plated overnight in T25 flasks and treated with 0.003 LDU/cell Ad-IU2, Ad-E4PSESE1a, 75 ng/ml rhTRAIL or PBS. 24 hours after 
infection, media were replaced with fresh media, and each treatment group was irradiated using a Gammacell 40 Cs-137 irradiator (MDS Nordion, Ottawa, ON, Canada) for a dose of $0,1,2,3$ or 4 Gy. 24 hours after irradiation, cells were washed with cold PBS, harvested, counted and reseeded at low densities into 5 P100 dishes for each treatment group. Cell seeding was adjusted for the relative plating efficiencies of each experimental group. Cells were maintained in culture for $\sim 3$ weeks (CWR22rv) and $\sim 2$ weeks (C4-2), changing the media once per week. Colony formation was monitored by light microscopy, and at the end of the incubation period, colonies were washed with cold PBS, stained with $0.5 \%$ crystal violet solution for 10 minutes and washed with cold tap water. Colonies of at least 50 cells were counted, and plating efficiencies were calculated as the number of colonies formed / the number of cells plated. Survival for each experimental group was calculated as the plating efficiency for the irradiated cells / the plating efficiency of non-irradiated cells within each treatment group. Survival data was analyzed on a logarithmic scale using the linear-quadratic model $\left[\ln \left(S F / S F_{0}\right)=-\alpha D\right.$ $\left.+\beta D^{2}\right]$ to determine the shape of the curve. The mean lethal dose $\left(D_{0}\right)$ was determined as the RT dose at which $37 \%$ of cells survive, and was compared to that of PBS for each treatment group to calculate the dose reduction factor (DRF).

\section{Infectivity Assay}

To achieve equal infectivity in non-prostatic bladder and colorectal cell lines, GFP expression was compared for these cells to that of prostate cancer cells following infection with Ad-GFP, a replication-deficient adenovirus expressing GFP under control of the CMV promoter. $7.5 \times 10^{4}$ CWR22rv, LoVo and T24 cells were plated overnight in 24-well plates and infected with Ad-GFP at doses ranging from 0 to $1000 \mathrm{vp} / \mathrm{cell}$ for CWR22rv, 0 to $5,000 \mathrm{vp} /$ cell for LoVo and 0 to 10,000 for T24 cells. 24 hours after infection, cells were washed with cold PBS, harvested, fixed with $2 \%$ paraformaldehyde, 
resuspended in PBS, and analyzed for GFP expression by FACS analysis. GFP expression of LoVo and T24 cell lines was compared to that of CWR22rv cells to determine relative infectivity, which was used in subsequent experiments.

\section{MTT Assay for In Vitro Cell Survival}

$1 \times 10^{4}$ LoVo and $7.5 \times 10^{3}$ T24 cells were cultured overnight in 96-well plates and infected with 0.2 LDU/cell (LoVo) or 4 LDU/cell (T24) Ad-IU2, Ad-E4PSESE1a or PBS. 24 hours after infection, media were changed and the cells were irradiated using a Nordion Gammacell 40 for a dose of 0, 1, 2, 3 or 4 Gy. Cells were maintained in culture, changing the media every other day. 96 hours after irradiation for LoVo cells or 48 hours after irradiation for T24 cells, cell survival was assessed by MTT assay. $25 \mu \mathrm{l}$ of $5 \mathrm{mg} / \mathrm{ml}$ MTT solution (3-(4,5-dimethylthiazol-2-yl)-2,5-diphenyltetrazolium bromide) was added to each well of cells containing $100 \mu$ of medium and incubated at $37^{\circ} \mathrm{C}$ for 3 hours. Following the incubation period, the media were removed, and the converted dye was solubilized with $75 \mu \mathrm{l}$ acidic isopropanol $(0.04 \mathrm{~N} \mathrm{HCl}$ in absolute 2-propanol). Absorbance of the reduced dye was analyzed by photospectroscopy at a wavelength of $570 \mathrm{~nm}$. Cell viability at each radiation dose was determined as the percentage of the $A_{570}$ value for each viral treatment group compared to the $A_{570}$ value for untreated (PBS), Ad-IU2- or Ad-E4PSESE1a-treated cells at 0 Gy.

\section{Measurement of Apoptosis Induction Following Irradiation of Cells}

$7.5 \times 10^{4}$ CWR22rv and C4-2 cells were cultured overnight in 24-well plates and infected with 0.003 LDU/cell Ad-IU2, Ad-E4PSESE1a or PBS. 24 hours after infection, media were replaced with fresh media, and the cells were irradiated using a Nordion Gammacell 40 for 0 or 3 Gy. 24 hours after irradiation, media and cells were harvested, washed with PBS and resuspended in $100 \mu$ l binding buffer. Cells were stained with 2.5 
$\mu$ l each of Annexin V-FITC and propidium iodide (PI) (BD Biosciences, Pharmingen) for 15 minutes at room temperature and analyzed by FACS analysis as above. Cells that were single-positive for Annexin V-FITC or double-positive for Annexin V-FITC and PI were considered as positive for apoptosis.

\section{Cell Cycle Analysis}

To determine the effect of Ad-IU2 infection on the cell cycle of PSA/PSMA prostate cancer cells, $1.5 \times 10^{5}$ CWR22rv and C4-2 cells were plated in 12-well plates overnight and treated with 0.003 LDU/cell Ad-IU2, Ad-E4PSESE1a, $100 \mathrm{ng} / \mathrm{ml} \mathrm{rhTRAIL}$ or PBS. 24 hours after treatment, the cells were washed with cold PBS, harvested and fixed with $2 \%$ paraformaldehyde. To determine the effect of Ad-IU2 infection on the cell cycle of irradiated prostate cancer cells, CWR22rv and C4-2 cells were treated as above. 24 hours after treatment, cells were irradiated with a dose of 0 or 3 Gy RT. 8,16 and 24 hours after irradiation, cells were washed with cold PBS, harvested and fixed with $2 \%$ paraformaldehyde. All cells were then permeabilized with $0.6 \%$ NP-40 detergent, treated with $1 \mathrm{mg} / \mathrm{ml}$ RNaseA and stained with $0.1 \mathrm{mg} / \mathrm{ml} \mathrm{PI}$ for 30 minutes. DNA content was analyzed by FACS analysis on a Beckton Dickinson Facscan flow cytometer. Cell cycle data was plotted as a histogram from FL2-area on WinMDI 2.9, and cell cycle calculations were performed by the standard algorithms within the Windows software, Cylchred. The percentage of cells in $G_{1}, S$ and $G_{2} / M$ following combined treatment was compared for all treatment groups.

\section{Statistical Analysis}

Statistical significance was determined using two-way ANOVA with Bonferroni's post-test or unpaired $t$ test, as indicated, using GraphPad Prism version 5.01 for Windows (GraphPad Software, San Diego, CA). Statistical significance was defined as 
a $P$ value $<0.05$, or better. Experiments were plated in triplicate, unless otherwise noted, and performed as at least three independent experiments. 


\section{Development of Ad-IU2 for Androgen-Independent Prostate Cancer}

\section{Background}

Current therapies for men presenting with localized prostate cancer include radical prostatectomy, external beam radiation therapy and brachytherapy; however, $25 \%$ of these men will experience local failure within ten years of treatment $(50,258)$. ADT is used for locally advanced and high risk prostate cancer, resulting in a slower rate of cancer growth and dissemination to distant sites; however, nearly all advanced prostate cancers eventually fail hormone therapy. Results from phase III clinical studies have recently suggested a role for docetaxel in the treatment of androgen-independent prostate cancer, demonstrating a two month survival advantage in addition to palliation $(259,260)$. Unfortunately, dose-limiting toxicities associated with such therapies limit the amount of the drug that can be delivered to the tumor, allowing the cancer to survive and fail therapy. Due to its ability to selectively target prostate cancer cells through the use of tissue-specific promoters and its widely demonstrated clinical safety profile, molecular therapy for androgen-independent prostate cancer is an attractive adjuvant to conventional therapies.

Tumor necrosis factor-related apoptosis-inducing ligand (TRAIL), also known as Apo-2 ligand, is a member of the tumor necrosis factor (TNF) family. Originally discovered because of its similarity to Fas-ligand, TRAIL is a $32 \mathrm{kDa}$ type II transmembrane protein, whose C-terminal extracellular domain (amino acids 114-281) is homologous to other members of the TNF family $(261,262)$. After binding of homotrimeric TRAIL to the death domain-containing receptors DR4 (263) and DR5 (264), the apoptotic signal is transduced via the adapter molecule, Fas-associated death domain (FADD), which recruits the initiator caspases to the death-inducing signaling complex (DISC) (265) (Figure 4). TRAIL has been shown to preferentially kill tumor cells 


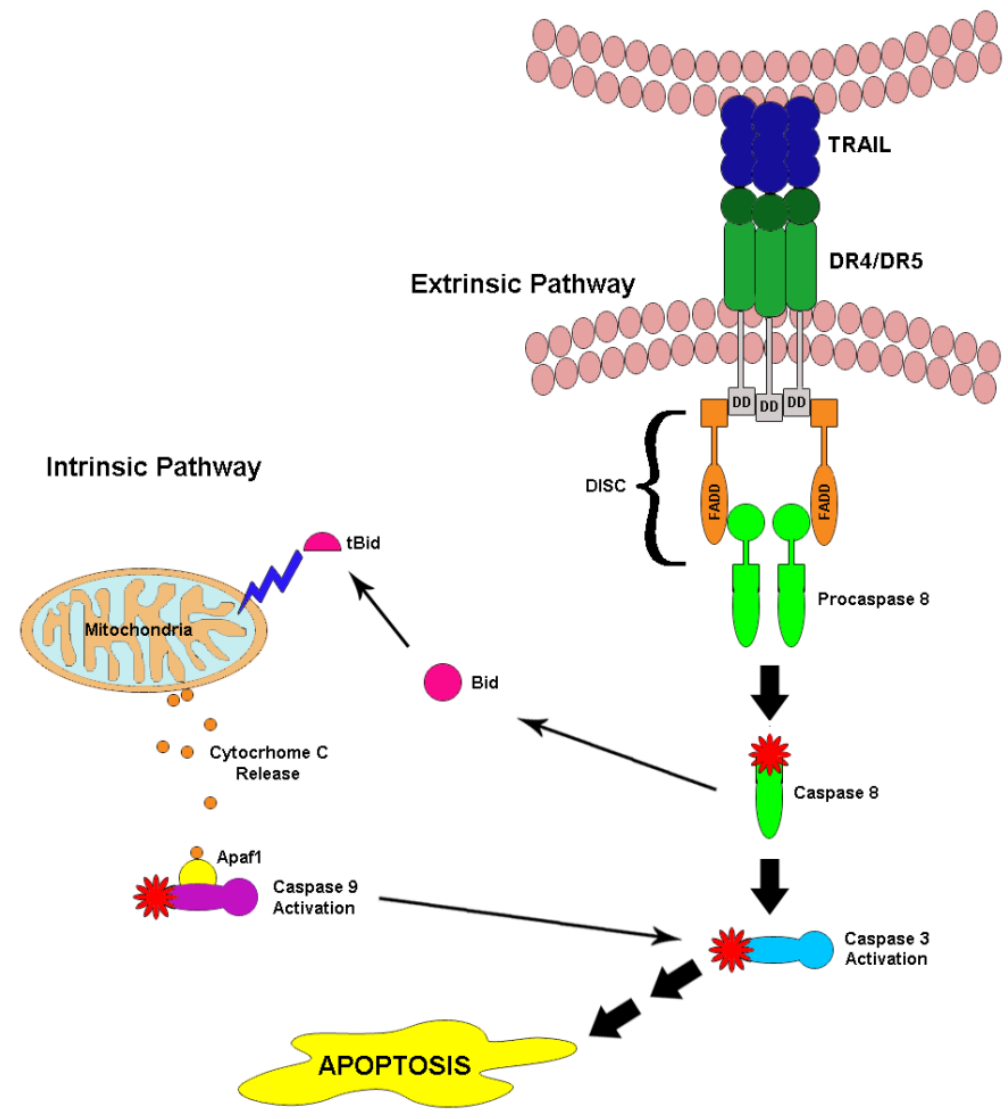

Figure 4. Induction of apoptosis by TRAIL via the extrinsic and intrinsic pathways. Trimerized TRAIL induces trimerization of the TRAIL receptors (DR4 or DR5). This in turn causes clustering of the death domains (DD) and association with the Fasassociated death domain (FADD), which serves as an adaptor molecule for

the death effector domain (DED) of the initiator caspases. Upon formation of the deathinducing signaling complex (DISC), procaspase 8 is recruited to the membrane and autocatalytically cleaved to its active state, caspase 8 . In the extrinsic pathway, the caspase cascade continues with activation of caspase 3 by caspase 8 . The apoptosis signal is amplified through a loop in the intrinsic pathway. Activated caspase 8 truncates Bid to tBid, which destabilizes the mitochondrial membrane, releasing cytochrome $\mathrm{C}$ from the cytosol. Cytochrome $\mathrm{c}$ binds to apoptotic protease activating factor 1 (Apaf1) which results in the cleavage and activation of caspase 9. This in turn further activates caspase 3 leading to enhanced apoptotic protease activation. 
over normal cells, which reflects its role as a key tumor immunosurveillance molecule in the body (266). This marked specificity for cancer cells gives TRAIL a distinct advantage over other cancer therapies.

TRAIL expression has been detected in several normal human tissues, suggesting that TRAIL is not toxic to those cells in vivo (263). These cells are shielded from TRAIL by the surface expression of antagonistic decoy receptors. Three nonapoptotic signaling receptors exist for TRAIL, and these include DcR1 which lacks an intracellular death domain (264), DcR2 which contains a truncated death domain that activates anti-apoptotic NFKB signaling (267), and osteoprotegrin, a secreted receptor for TRAIL which also inhibits osteoclastogenesis (268). Several prostate cancer cell lines including ALVA-31, DU-145 and PC-3 are extremely sensitive to TRAIL and undergo apoptosis when exposed; however, other cell lines such as LNCaP are highly resistant (269). This resistance has been shown to be reversed by infection with adenovirus (270), treatment with chemotherapeutic agents such as paclitaxel, vincristine, etoposide, doxorubicin or camptothecin (271), or treatment of the cells with radiation therapy $(272)$.

Recent preclinical and clinical studies of molecular therapy approaches with TRAIL have involved a soluble form of the protein that is secreted out of producer cells and into the surrounding tumor matrix. This strategy proved advantageous over the repeated intravenous administration of recombinant TRAIL protein, as the pharmacologic half-life of the protein in serum is only 32 minutes (273). Although repeated administration of soluble TRAIL was not toxic to normal tissues in mice (274) and non-human primates (273), recent data suggest that cultured human hepatocytes may be sensitive to soluble forms of $\operatorname{TRAIL}(275,276)$. To enhance the safety and clinical feasibility of this gene therapy strategy for high risk prostate cancer, I developed a PSRCA, called Ad-IU2, encoding full-length membrane-bound TRAIL under the 
transcriptional control of PSES. Furthermore, to limit the replication of Ad-IU2 to prostate cancer cells, adenoviral E1a, E1b and E4 genes were placed under control of PSES. As PSES is active only in PSA/PSMA-positive cells, adenoviral replication and TRAIL expression should be limited to PSA/PSMA-positive cells. To date, this is the first prostate-specific promoter-driven TRAIL molecular therapeutic strategy for advanced prostate cancer. In this portion of my thesis, I test the hypothesis that the addition of the cytotoxic transgene TRAIL will enhance the in vitro and in vivo anti-tumor activity of a replication-competent adenovirus against androgen-independent prostate cancer.

\section{In Vitro and In Vivo Characterization of Ad-IU2}

Ad-IU2 Delivered Surface-Bound TRAIL and Replicated Effectively in PSA/PSMAPositive Cells

The structure of Ad-IU2 (Figure 5) is based on the PSRCA, Ad-E4PSESE1a, in which the E1 promoter was deleted and E1a moved to the right ITR E4 region under control of the bidirectional PSES enhancer sequence (205). Full-length, membranebound TRAIL CDNA was inserted at the left ITR in the E1a region upstream from

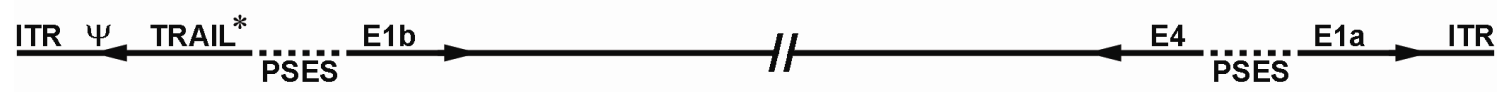

Figure 5. Genomic structure of Ad-IU2. TRAIL cDNA was cloned into the left ITR under control of the bidirectional PSES enhancer. To avoid interference with the adenoviral packaging sequence $(\psi)$, E1a was placed at the right ITR under the transcriptional control of PSES along with E4. Replication competent adenoviral control vector, Ad-IU1 was constructed by replacing the PSES-TRAIL cassette with a PSES-HSV-TK expression cassette $\left(^{*}\right)$. 
adenoviral E1b, both under the control of PSES. As depicted in Figure 6, full-length TRAIL protein expression was confirmed by western blot in Ad-IU2-infected PSA/PSMApositive CWR22rv prostate cancer cells. Immunoblot of Ad-IU2-infected cells revealed a $32 \mathrm{kD}$ band comparable in size to that of full-length TRAIL expressed in pORF-hTRAILtransfected cells. Infection with Ad- $\triangle T A T A-E 1 a$ confirmed the lack of endogenous TRAIL expression or the upregulation of TRAIL by adenoviral infection in CWR22rv cells.

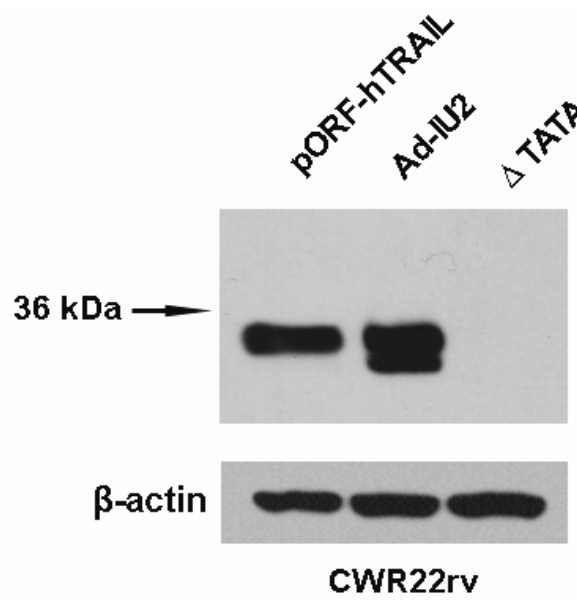

Figure 6. Expression of TRAIL in PSA/PSMA-positive CWR22rv cells was confirmed by western blot. CWR22rv cells were infected with $0.01 \mathrm{LDU} / \mathrm{cell}$ Ad-IU2 or Ad- $\triangle T A T A-E 1 a$ (negative vector control). As a positive control, cells were transfected with pORF-hTRAIL. Forty-eight hours after infection or transfection, cells lysates were harvested and $20 \mu \mathrm{g}$ of sample were separated by $10 \%$ SDS-PAGE. The blot was probed with anti-human TRAIL antibody. Full length monomer TRAIL was detected in the lysates of Ad-IU2-infected and pORF-hTRAIL-transfected CWR22rv cells at approximately $32 \mathrm{kDa}$. The double band detected in Ad-IU2-infected lysates demonstrates a commonly observed phenomenon in our laboratory, likely due to alternative ATG sites in adenoviral-expressed transgenes. No endogenous TRAIL expression was detected following infection with Ad- $\triangle T A T A-E 1 a$.

To confirm that TRAIL was expressed on the cellular membrane of infected cells, FACS analysis for cell-surface expression of TRAIL was performed. Infection of CWR22rv 
prostate cancer cells with Ad-IU2 resulted in a significant enhancement of TRAIL surface expression $(28.6 \%$ vs. $2.7 \%, p<0.001)$, compared to infection with the non-TRAIL expressing vector, Ad-IU1 (Figure 7).

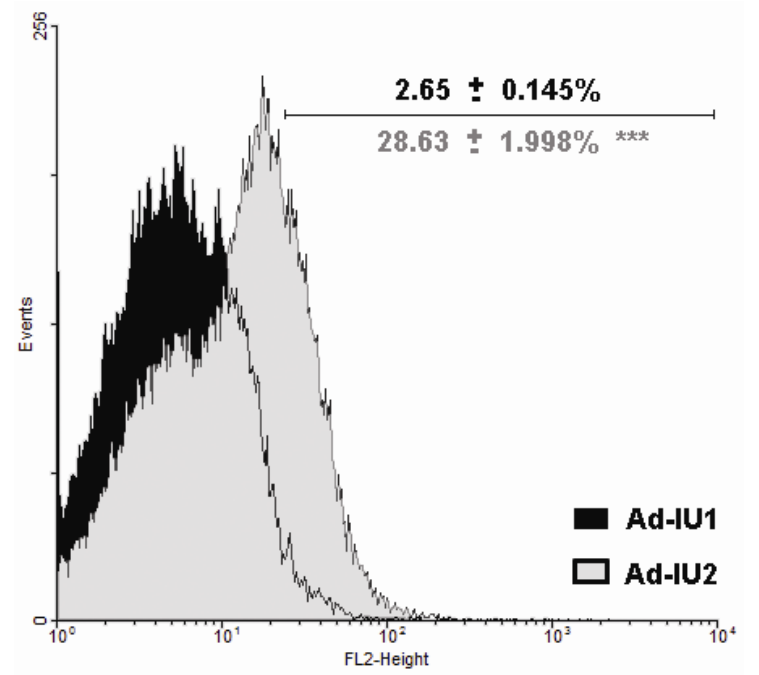

Figure 7. Cell surface expression of TRAIL was confirmed in CWR22rv prostate cancer cells following infection with Ad-IU2. CWR22rv cells were infected with $0.01 \mathrm{LDU} /$ cell Ad-IU2 or Ad-IU1. Forty-eight hours after infection, cells were harvested and stained with PE-conjugated anti-human TRAIL antibody for one hour and analyzed for cell surface expression by FACS. Membrane-bound TRAIL was detected in Ad-IU2-infected CWR22rv cells.

Previous studies have demonstrated that adenoviral vectors expressing apoptosis-inducing transgenes and death ligands replicate poorly due to decreased adenoviral gene expression and producer cell toxicity. This results in decreased replication efficiencies, low production yields and poor transduction efficiencies (277). Expression of TRAIL protein in cells may be inversely proportional to the ability of the virus to replicate in those cells; therefore, I performed a replication assay in prostate cancer cell lines to determine whether Ad-IU2 replication efficiency was inhibited by TRAIL expression. Ad-IU2 replicated as efficiently as the PSRCA, Ad-E4PSESE1a, in PSA/PSMA-positive cells; however, Ad-IU2 failed to propagate in PSA/PSMA-negative cells, resulting in viral output yields comparable to that of the replication-deficient virus, 
Ad- $\triangle$ TATA-E1a (Table 4). These results demonstrate that Ad-IU2 replication was not hindered by the expression of TRAIL in PSA/PSMA-positive prostate cancer cells. Because control of viral replication was achieved through prostate-specific transcriptional regulation of adenoviral early genes, including E1a, I tested whether E1a protein expression was limited to PSA/PSMA-positive cells. As demonstrated in Figure 8, E1a protein was expressed in all cell lines following infection with Ad-wt. On the other hand, after infection with Ad-IU2, E1a protein expression was restricted to PSA/PSMApositive C4-2 and CWR22rv cells. These data are consistent with the results from the propagation assay, suggesting that the selectivity of Ad-IU2 replication for PSA/PSMA-

Table 4. Ad-IU2 replicated efficiently in and restricted to PSA/PSMA-positive prostate cancer cells. 1 x 106 PSA/PSMA-positive (CWR22rv, C4-2 and LNCaP) and PSA/PSMA-negative (PC-3 and DU-145) cells were infected with the indicated input dose of either Ad-ATATA-E1a, Ad-E4PSESE1a or Ad-IU2. Viral supernatants were harvested 72 hours post-infection and applied to HER911E4 producer cells at serial dilutions ranging from 1 to $10^{-11}$. Cytopathic effect was examined by light microscopy, and $\mathrm{LD}_{50}$ was calculated as the greatest viral dilution factor producing a cytopathic effect in at least 4 of 8 wells. ${ }^{*}$ Replication-deficient negative control. ${ }^{* *}$ Replication-competent negative control.

\begin{tabular}{|c|c|c|c|c|}
\hline \multirow{2}{*}{ Cell Line } & \multirow{2}{*}{$\begin{array}{l}\text { Input Dose } \\
\text { (LDU) }\end{array}$} & \multicolumn{3}{|c|}{ Output Viral Dose $\left(L D_{50}\right)$} \\
\hline & & $\Delta$ TATA $^{*}$ & Ad-E4PSESE1a** & Ad-IU2 \\
\hline CWR22rv & $1 \times 10^{4}$ & $3.4 \times 10^{2}$ & $3.7 \times 10^{6}$ & $3.7 \times 10^{6}$ \\
\hline C4-2 & $1 \times 10^{4}$ & $7 \times 10^{1}$ & $4 \times 10^{5}$ & $4 \times 10^{5}$ \\
\hline LNCaP & $1 \times 10^{4}$ & $7 \times 10^{1}$ & $7 \times 10^{5}$ & $3.7 \times 10^{6}$ \\
\hline PC-3 & $1 \times 10^{5}$ & $7 \times 10^{1}$ & $4 \times 10^{1}$ & $4 \times 10^{1}$ \\
\hline DU-145 & $1 \times 10^{5}$ & $7 \times 10^{1}$ & $7 \times 10^{1}$ & $7 \times 10^{1}$ \\
\hline
\end{tabular}


positive prostate cancer cells was due to limited expression of adenoviral early genes in PSA/PSMA-negative cells.

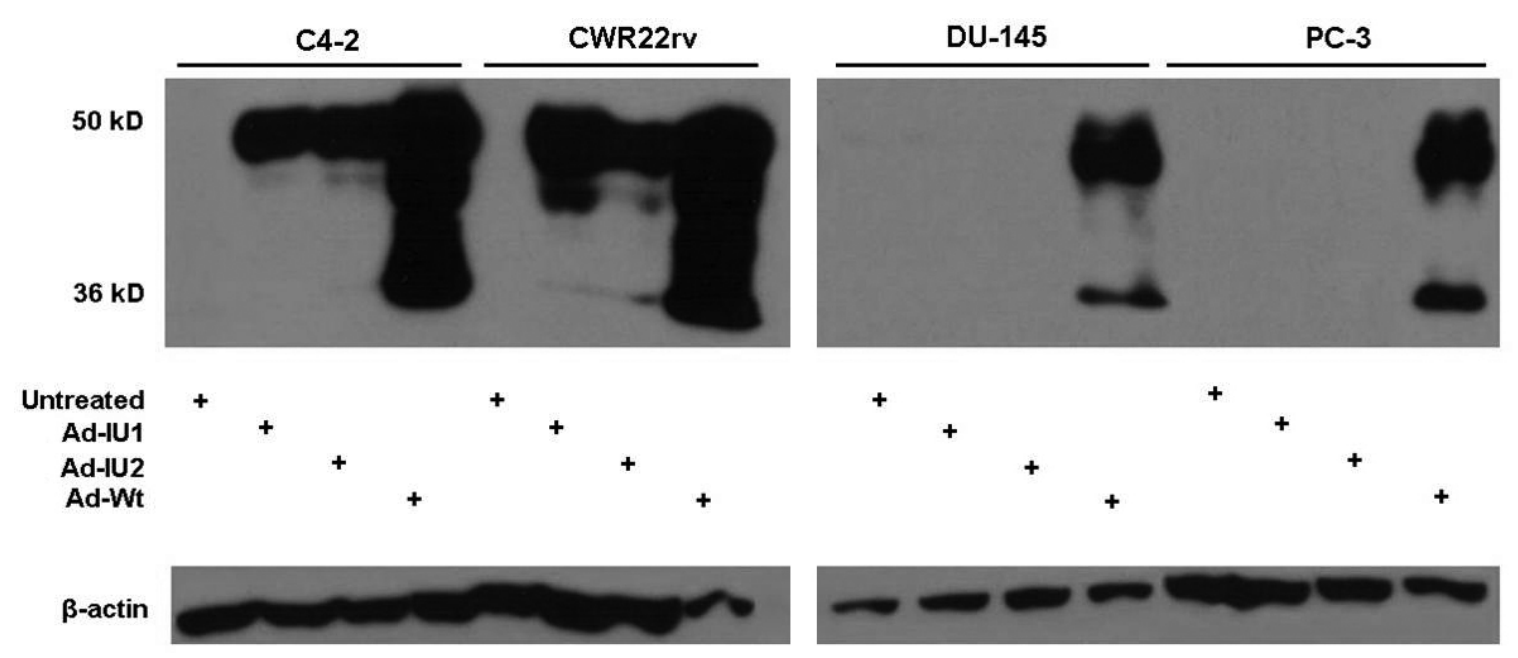

Figure 8. PSES-driven expression of adenoviral E1a was limited to PSA/PSMA-positive cells. PSA/PSMA-positive C4-2 and CWR22rv cells were infected with $100 \mathrm{vp} / \mathrm{cell}$ and PSA/PSMA-negative DU-145 and PC-3 cells were infected with 1000 vp/cell Ad-IU1 (PSRCA control), Ad-IU2 or Ad-Wt. Forty-eight hours after infection or transfection, cells lysates were harvested and $20 \mu \mathrm{g}$ of sample were separated by $10 \%$ SDS-PAGE. The blot was probed with anti-Ad5 E1a antibody. Adenoviral E1a proteins were detected by western blot from $36 \mathrm{kD}$ to $50 \mathrm{kD}$. E1a expression, which controls adenoviral replication, was limited to PSA/PSMA-positive prostate cancer cells.

\section{Ad-IU2 Effectively Induced Apoptosis and Reversed Resistance in PSA/PSMA- Positive Prostate Cancer Cells}

Apoptosis-inducing agents such as TRAIL have shown promising clinical potential against solid tumors $(83,270,278)$; therefore, I tested the ability of Ad-IU2 to induce apoptosis in prostate cancer cell lines. PSA/PSMA-positive prostate cancer cells, CWR22rv, C4-2 and LNCaP, as well as PSA/PSMA-negative cell lines, PC-3 and DU- 
145 were treated with PBS, Ad-IU2 or Ad-IU1 for 24 hours and analyzed for apoptosis by FACS analysis. As depicted in Figure 9, apoptosis induction within 24 hours of Ad-IU2

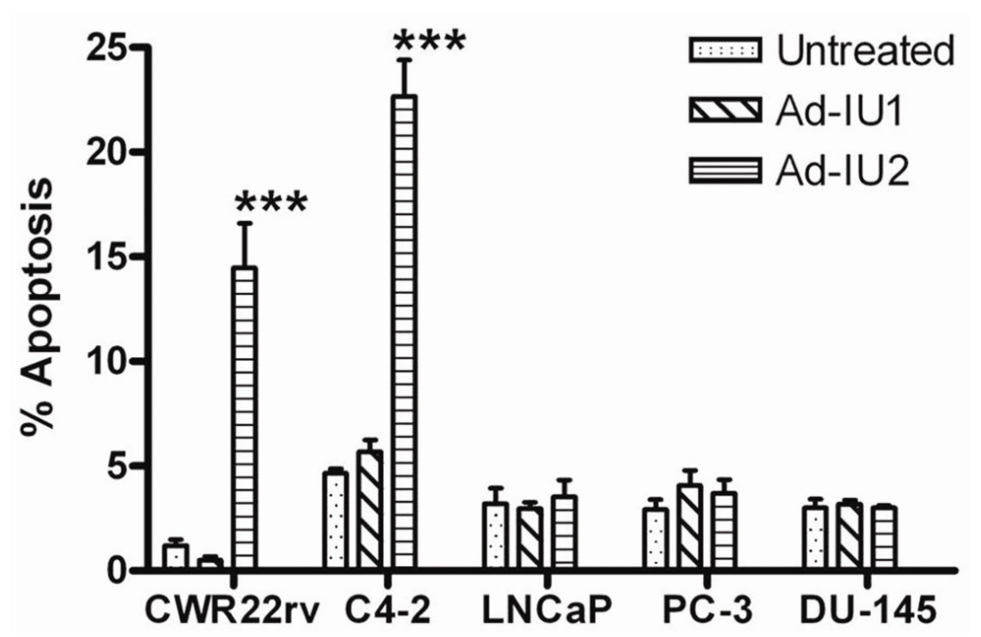

Figure $\quad 9 . \quad$ Apoptosis induction by Ad-IU2. CWR22rv, C4-2, LNCaP, PC-3 and DU-145 cells were plated overnight in a 24-well plate and treated with PBS or $0.01 \mathrm{LDU} / \mathrm{cell}$ Ad-IU1 or AdIU2. Twenty-four hours after

Infection, media and cells were harvested, stained with Annexin V-FITC and PI and analyzed by FACS. Ad-IU2 induced 5 -fold greater apoptosis at 24 hours than the PSRCA control, Ad-IU1, specifically in PSA/PSMA-positive prostate cancer cells. PSA/PSMA-positive LNCaP cells were resistant to apoptosis induction at 24 hours. ${ }^{* * *}=$ $\mathrm{p}<0.001$ difference between Ad-IU2 and Ad-IU1.

infection in CWR22rv and C4-2 cells was nearly 5-fold higher than baseline or Ad-IU1induced levels. As expected, no apoptosis above baseline was detected in PSA/PSMAnegative PC-3 and DU-145 prostate cancer cell lines. Likewise, no apoptosis was detected in the PSA/PSMA-positive LNCaP cell line, which has been shown to be highly resistant to TRAIL-mediated apoptosis due to high AKT activity (269). This TRAIL resistance was overcome however, by prolonged infection with Ad-IU2. LNCaP cells infected with Ad-IU2 for 48 hours demonstrated greater than 5-fold induction of apoptosis above that of control virus infection (Figure 10). This is consistent with 
previous reports demonstrating that TRAIL resistance can be overcome by coexpression of TRAIL and adenoviral E1a (279).

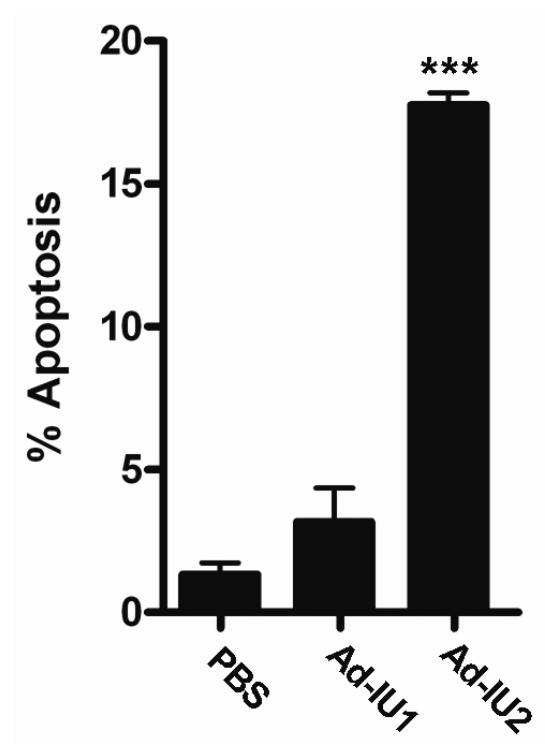

Figure 10. TRAIL resistance was reversed in LNCaP cells following prolonged viral infection. LNCaP cells were treated with PBS, 0.01 LDU/cell Ad-IU1 or Ad-IU2 for 48 hours. Infection of TRAIL-resistant PSA/PSMA-positive LNCaP cells with Ad-IU2 for 48 hours reversed resistance to TRAIL-mediated apoptosis. $\quad{ }^{* * *}=\mathrm{p}<0.001$ difference between Ad-IU2 and Ad-IU1.

\section{Ad-IU2 Effectively Killed Prostate Cancer Cells, While It Spared Normal Cells}

To assure that apoptosis induction and viral replication within cells was sufficient to kill prostate cancer cells, an in vitro killing assay was performed on CWR22rv, C4-2, LNCaP and adult human dermal fibroblast (HDFa) cells. Following treatment with PBS or serial dilutions of Ad-IU2, Ad-E4PSESE1a or Ad- $\triangle$ TATA-E1a (replication-defective adenoviral control), cytopathic effect of the virus was monitored by light microscopy and viral killing was determined by staining attached cells with crystal violet. The oncolytic effect was observed earliest in C4-2 and LNCaP cells, resulting in assay end-points of 3 days post-infection for C4-2 and LNCaP and 4 days post-infection for CWR22rv. This difference in time course between prostate cancer cell lines is consistent with delayed replication and transgene expression due to slightly reduced PSES transcriptional activity in CWR22rv cells (124). Ad-IU2 exhibited greater killing efficiency in PSA/PSMA-positive prostate cancer cells at doses markedly lower than the PSRCA, Ad- 

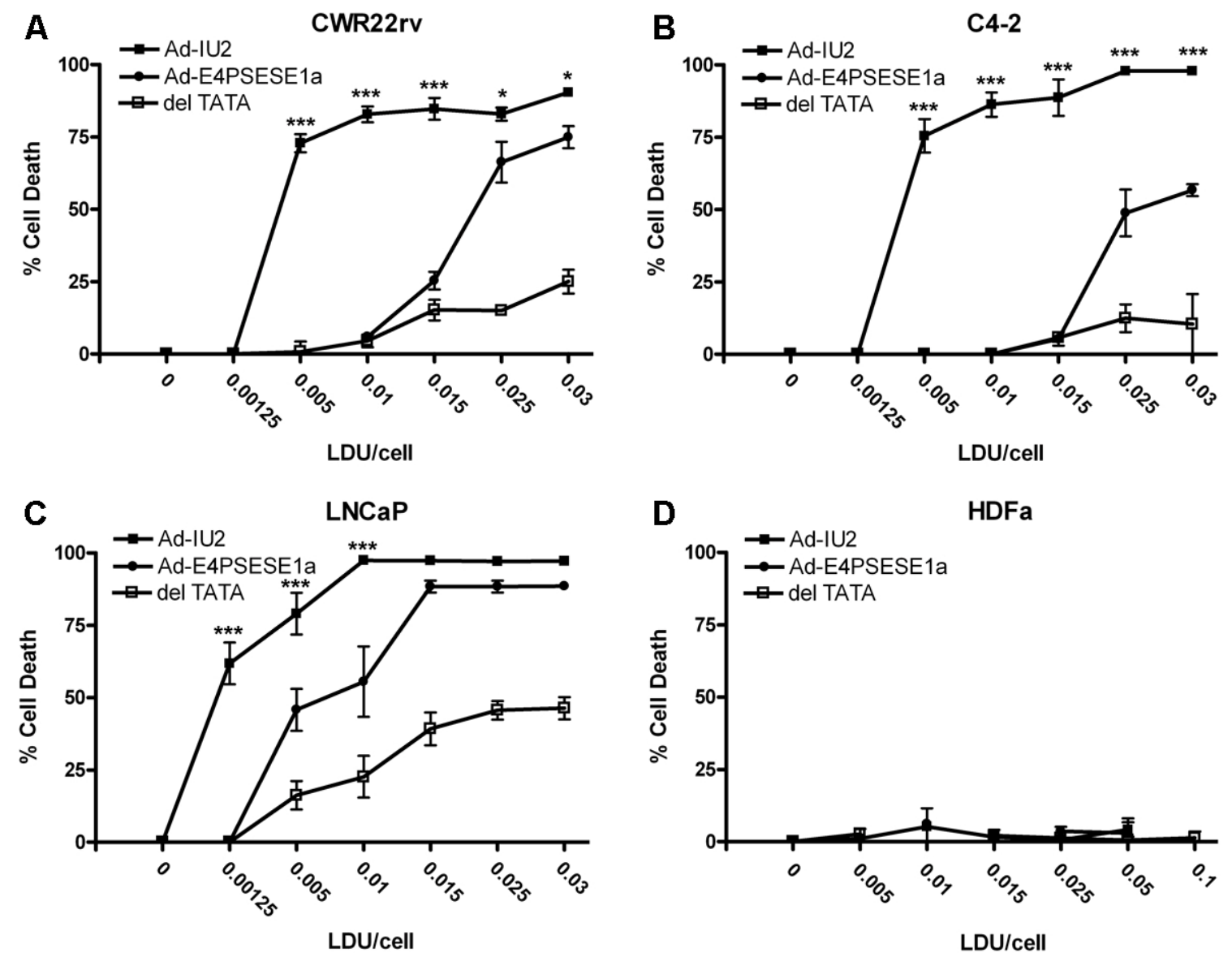

Figure 11. Ad-IU2 induced marked in vitro cell killing of PSA/PSMA-positive prostate cancer cells. Cells were plated in 24-well plates and treated with various doses of AdIU2, Ad-E4PSESE1a (replication-competent control), Ad- $\triangle T A T A-E 1 a$ (replicationdeficient control) or PBS. Crystal violet survival curves for CWR22rv (A), C4-2 (B), LNCaP (C) and human dermal fibroblasts (D). Ad-IU2 induced marked cell killing above that of Ad-E4PSESE1a at earlier time points specifically in PSA/PSMA-positive prostate cancer cells. Viability was assayed once cytopathic effect was detected by light microscopy at 4 days (CWR22rv), 3 days (C4-2 and LNCaP) and 7 days post-infection (HDFa, cytopathic effect was not detected). \% cell death was calculated as the ratio of $A_{570}$ for viral-treated cells to that of PBS-treated cells. ${ }^{*}=p<0.05,{ }^{* * *}=p<0.001$ difference between Ad-IU2 and Ad-E4PSESE1a. 
E4PSESE1a. As depicted in Figure 11A-C, the dose of Ad-IU2 required to kill at least 50\% of CWR22rv, C4-2 and LNCaP cells was 5-, 6- and 8-fold lower than that of AdE4PSESE1a, respectively. To demonstrate specificity for PSA/PSMA-positive cells, this experiment was repeated using normal human fibroblasts. Despite treatment of cells for a longer period of time, Ad-IU2 produced no cytotoxicity in HDFa cells (Figure 11D). These data suggest that expressing TRAIL in a PSRCA enhanced its anti-tumor cytotoxicity, while maintaining its PSA/PSMA-specificity.

\section{Ad-IU2 Demonstrated a Bystander Effect in PSA/PSMA-Negative Prostate Cancer Cells}

Due to limited viral transduction efficiency in vivo and the heterogeneity of human prostate tumors with regards to PSA/PSMA-expression, the ability to target and destroy prostate cancer cells in which a PSRCA cannot replicate and lyse the cell is critical to prevent the development of foci of untreated cells within a tumor. The killing power of Ad-IU2 might be enhanced through cell-to-cell contact of neighboring cells with infected prostate cancer cells or cell contact with the apoptotic bodies from dying cells. To determine whether Ad-IU2 imparted a bystander killing effect on neighboring PSA/PSMA-negative prostate cancer cells, I co-cultured Ad-IU1- or Ad-IU2-infected CWR22rv cells with mRFP-stably transfected PC-3 cells and detected the level of apoptosis induction in the mRFP-labeled PC-3 cells. As depicted in Figure 12, PC-3 cells, which failed to undergo apoptosis following direct infection with Ad-IU2 (Figure 9), exhibited a 4-fold induction of apoptosis above the level induced by Ad-IU1 co-culture when co-cultured with Ad-IU2-infected CWR22rv cells.

To determine whether direct cell-to-cell contact was necessary to produce a bystander killing effect, I tested the ability of conditioned media from Ad-IU2-infected cells to elicit a similar response. Conditioned media collected from CWR22rv cells 


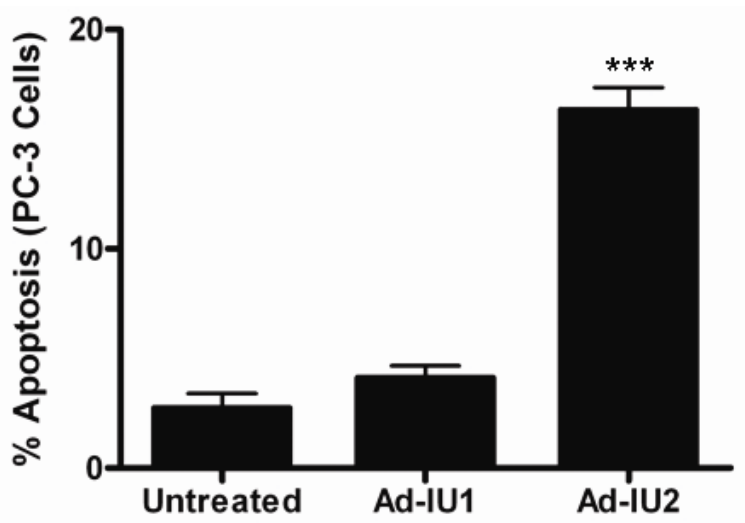

Figure 12. Ad-IU2 demonstrated a bystander effect in PSA/PSMA-negative cells in co-culture with infected PSA/PSMA-positive cells. CWR22rv cells were infected with $0.01 \mathrm{LDU} /$ cell Ad-IU1 or Ad-IU2. Twenty-four hours post-infection, cells were washed three times with PBS and mRFP-labeled PSA/PSMA-negative PC-3 cells were co-plated at a ratio of 3 CWR22rv cells to 1 PC-3 cell. After 24 hours in co-culture, media and cells were harvested, and the percent apoptotic mRFP-labeled PC-3 cells was analyzed by FACS analysis. A significant level of apoptosis was induced in PC-3 cells co-cultured with Ad-IU2-infected cells.

infected with Ad-IU2 or control virus were heat-treated to inactivate any adenoviral particles present. When treated with conditioned media, both CWR22rv and PC-3 cells achieved a similar level of apoptosis induction (Figure 13). To assure that this effect was not directly mediated by adenoviral infection and that adenovirus was inactivated effectively by heat treatment, CWR22rv cells were treated with conditioned media from Ad-E4PSESE1a-infected CWR22rv cells. Heat inactivation of the conditioned media resulted in a 40-fold reduction in GFP-positivity compared to conditioned media without heat inactivation (Figure 14), demonstrating effective inactivation of adenovirus by heat treatment of the conditioned media. These results suggest that unidentified soluble factors can mediate the Ad-IU2 bystander effect. This in turn would enhance distribution of the cytotoxic effects throughout the entire tumor, as direct cell-to-cell contact is not required. To determine whether soluble TRAIL was cleaved from the cell membranes of infected cells and contributed to the bystander killing of prostate cancer cells, I 


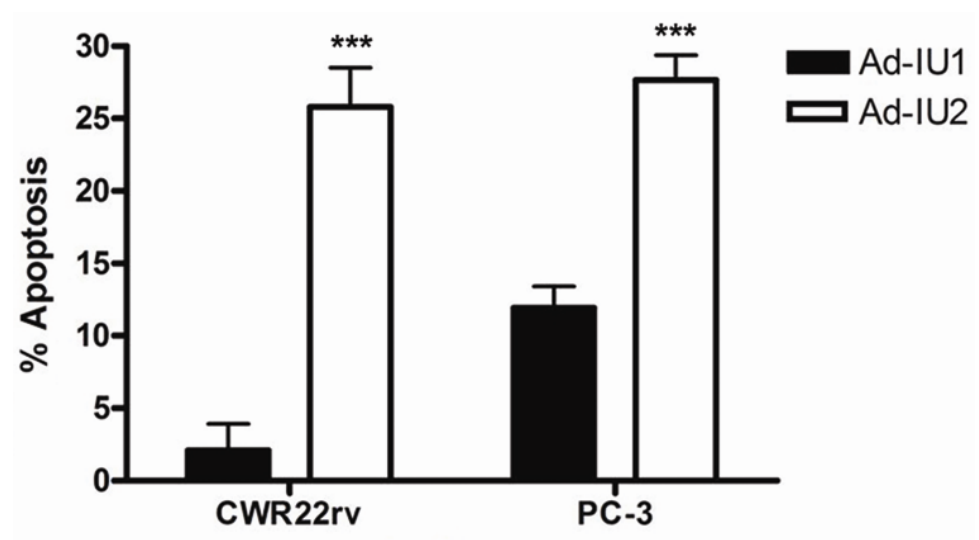

Figure 13. Heat-inactivated conditioned media from AdIU2-infected CWR22rv cells induced significant levels of apoptosis in PSA/PSMAnegative prostate cancer cells. CWR22rv cells were infected with $0.01 \mathrm{LDU} /$ cell Ad-IU1 or Ad-IU2. Forty-eight hours after infection, media were harvested and heat-inactivated at $56^{\circ} \mathrm{C}$ for 30 minutes. Conditioned media were applied to CWR22rv or PC-3 cells for 24 hours. Marked induction of apoptosis was achieved in PSA/PSMA-positive CWR22rv and PSA/PSMA-negative PC3 cells following treatment with Ad-IU2-infected conditioned media. ${ }^{* \star *}=p<0.001$.
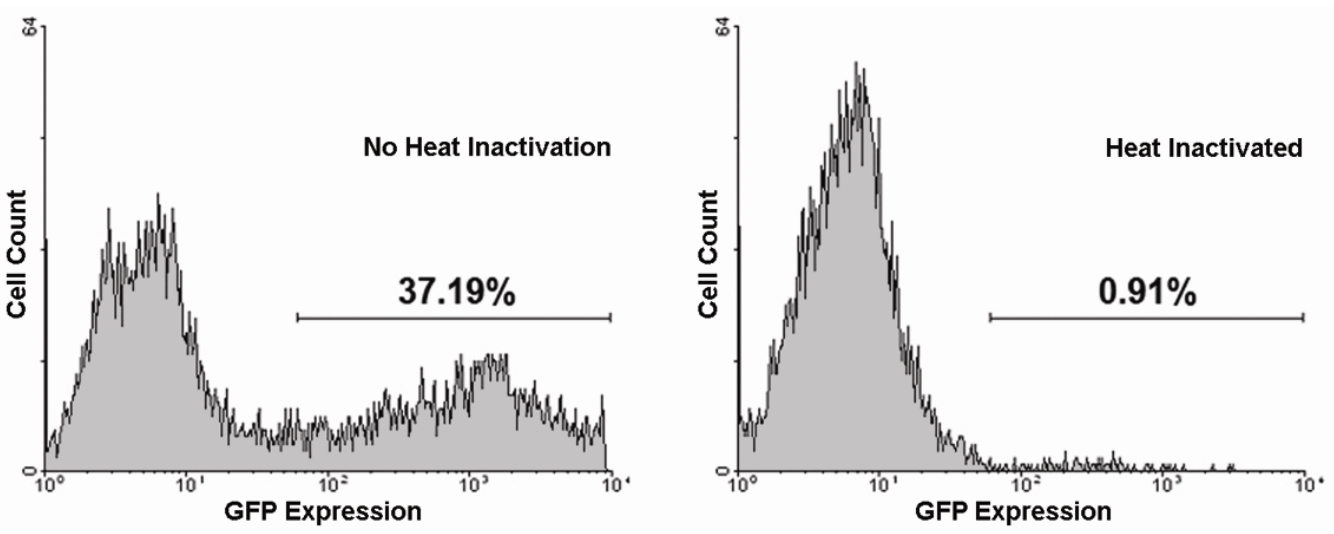

Figure 14. Heat treatment of conditioned media was sufficient to inactivate adenovirus. Media from CWR22rv cells infected with 0.01 LDU/cell Ad-E4PSESE1a for 48 hours was harvested and heat-inactivated at $56^{\circ} \mathrm{C}$ for 30 minutes. Conditioned media was applied to CWR22rv cells for 24 hours before or after heat-inactivation. Heat inactivation of conditioned media from Ad-E4PSESE1a-infected cells resulted in a drastic reduction in GFP-positive CWR22rv cells, demonstrating significant inactivation of adenovirus. 
performed western blot analysis on conditioned media from Ad-IU2-infected CWR22rV cells for TRAIL protein. As shown in Figure 15, cell lysate from Ad-IU2-infected CWR22rv cells expressed full-length, 32 kD TRAIL protein; however, no band was detected in the conditioned media from these cells. As a control, various dilutions of rhTRAIL were immunoblotted from 100 to $25 \mathrm{ng} / \mathrm{ml}$, and the intensity of the $18 \mathrm{kD}$ bands decreased with respect to protein concentration. These results confirm that soluble TRAIL was not secreted or cleaved from Ad-IU2 infected cells at concentrations suitable to induce cytotoxicity in prostate cancer cells.

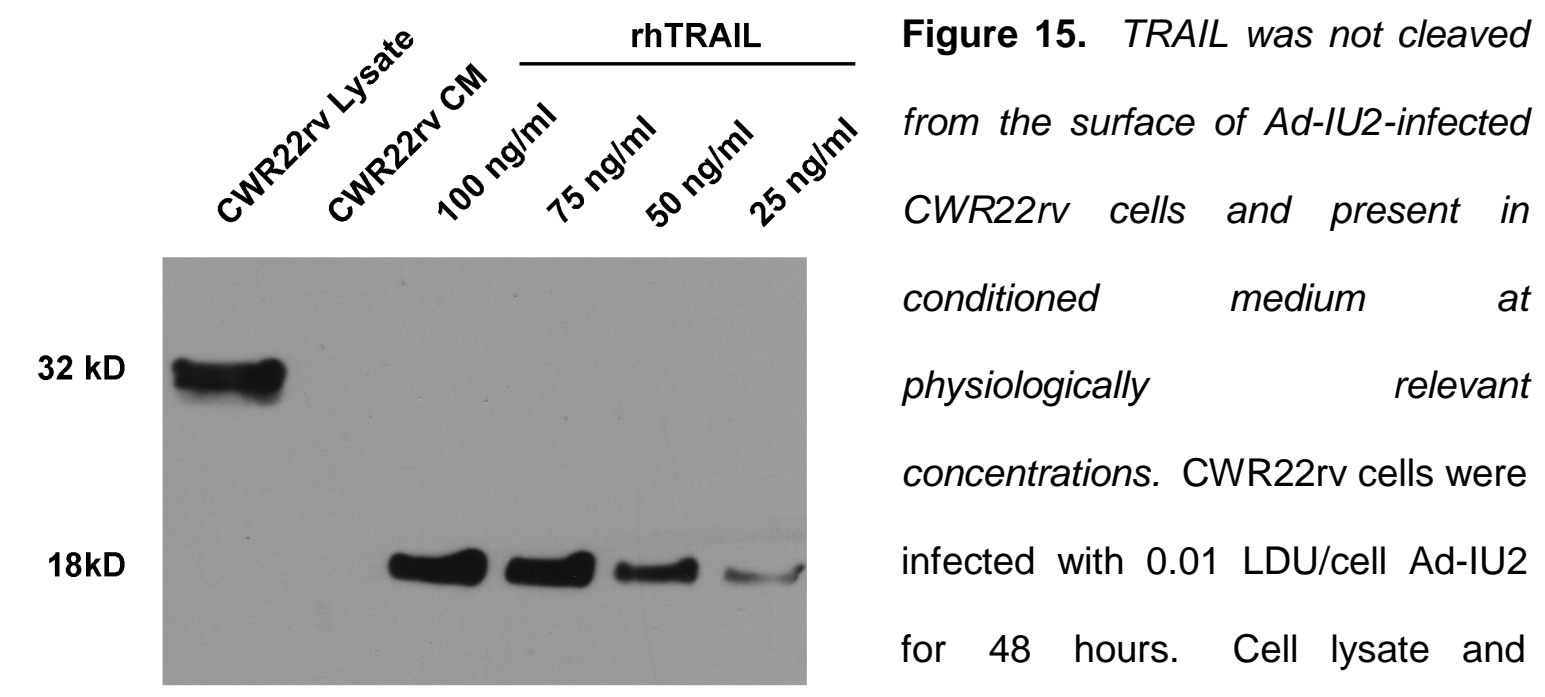

conditioned medium (CM) were harvested. Cell lysate, $\mathrm{CM}$ and various concentrations of rhTRAIL were separated by $12 \%$ SDS-PAGE and immunoblotted with anti-human TRAIL antibody. Soluble TRAIL was not detected in the CM from Ad-IU2-infected cells.

\section{Ad-IU2 Inhibited the Growth of SQ Androgen-Independent CWR22rv Xenografts}

Previously, we investigated the oncolytic potential of Ad-E4PSESE1a, a PSRCA, which significantly inhibited the growth of CWR22rv xenografts as compared to control virus; however, the response only lasted two weeks, after which the tumor growth 
exceeded the rate of oncolysis. Rapid intratumoral viral replication and spread peaked at 3 days and was diminished by 1 week after injection (205). For this reason, I determined whether TRAIL could augment the in vivo antitumor effects of a PSRCA. Androgen-independent CWR22rv human prostate cancer xenografts were established SQ in the flanks of castrated athymic male mice and injected with Ad-IU2, Ad-IU1 (replication-competent control) and PBS (vehicle control). As shown in Figure 16, AdIU2 significantly suppressed the growth of CWR22rv tumor xenografts as compared to Ad-IU1 (3.1- vs. 17.1-fold growth of tumor, respectively). 4 weeks after treatment, AdIU1-treated tumors began to fail therapy, resulting in a rebound of tumor growth. On the other hand, Ad-IU2 continued to inhibit tumor growth through the 6-week end-point of the

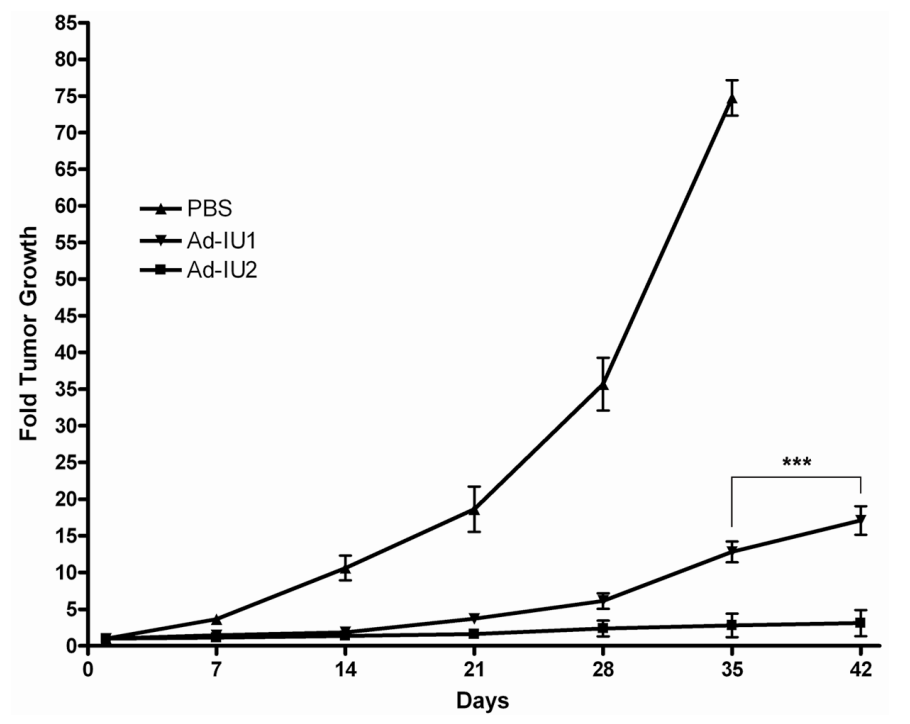

Figure 16. Ad-IU2 suppressed the growth of androgenindependent human prostate tumors in athymic mice. SQ androgen-independent CWR22rv xenografts were established in castrated male athymic mice and treated with intratumoral injections of $2 \times 10^{4}$ LDU Ad-IU1 $(n=6)$, Ad-IU2 $(n=9)$ or PBS $(n=5)$. Tumor volumes were calculated as $\left(L^{2} \times W\right) /(\pi / 6)$. Four weeks after treatment, Ad-IU1-treated tumors began to fail therapy, resulting in rebound of tumor growth. PBS-treated mice were sacrificed at 5 weeks due to tumor burden. Ad-IU2 significantly suppressed the growth of CWR22rv tumors as compared to Ad-IU1 (3.1-vs. 17.1-fold growth, respectively). ${ }^{* * *}=p<0.001$ (Ad-IU2 vs. Ad-IU1). 
study. Mock-treated mice were sacrificed at 5 weeks due to overwhelming tumor burden. Of the nine tumors treated with Ad-IU2, six responded favorably with partial regression in four of six or complete regression in two of six tumors. Of the three tumors that failed to respond, two were significantly suppressed compared to Ad-IU1-treated tumors at 6-weeks (Figure 17). Given the fact that CWR22rv xenografts are clonogenic, the variation in treatment outcome may be attributable to incomplete infiltration of the tumor or leakage at the time of injection. Histological examination of PBS-treated

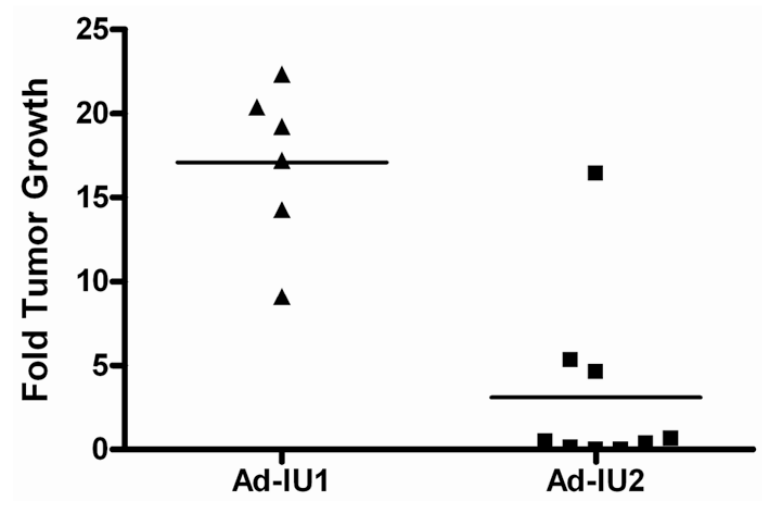

Figure 17. Individual fold tumor growth at the 6-week study endpoint. Of 9 AdIU2-treated tumors, 6 responded favorably with partial regression in 4 of 6 or complete regression in 2 of 6 tumors. Of the 3 tumors that failed, two were significantly suppressed compared to Ad-IU1-treated tumors at 6 weeks.

tumors revealed healthy cells arranged in normal tumor architecture with significant tumor vasculature in the margins of the growing tumor (Figure 18A). Ad-IU1-treated tumors were characterized by scattered necrotic patches surrounded by healthy tumor cells, indicative of incomplete oncolysis due to limited viral replication and propagation throughout the entire tumor mass (Figure 18B). Although patches of healthy tumor cells remained within the Ad-IU2-treated tumors, necrotic centers of viral replication and oncolysis were more diffuse throughout the entire tumor. Furthermore, cells immediately surrounding the necrotic centers appeared unhealthy with condensed nuclei, indicating spread of the cytotoxic and apoptotic effect beyond the necrotic centers (Figure 18C). 
To determine whether apoptosis contributed significantly to the tumor killing process, in situ TUNEL assays were performed on the tumor sections. No apoptotic nuclei were detected in the control tumors (Figure 19A,B). On the other hand, Ad-IU2-treated tumors displayed marked apoptosis in the margins surrounding necrotic centers of oncolysis (Figure 19C). These data suggest that TRAIL potentiated the in vivo killing power of a PSRCA through apoptosis induction in cells beyond the margin of viral replication.
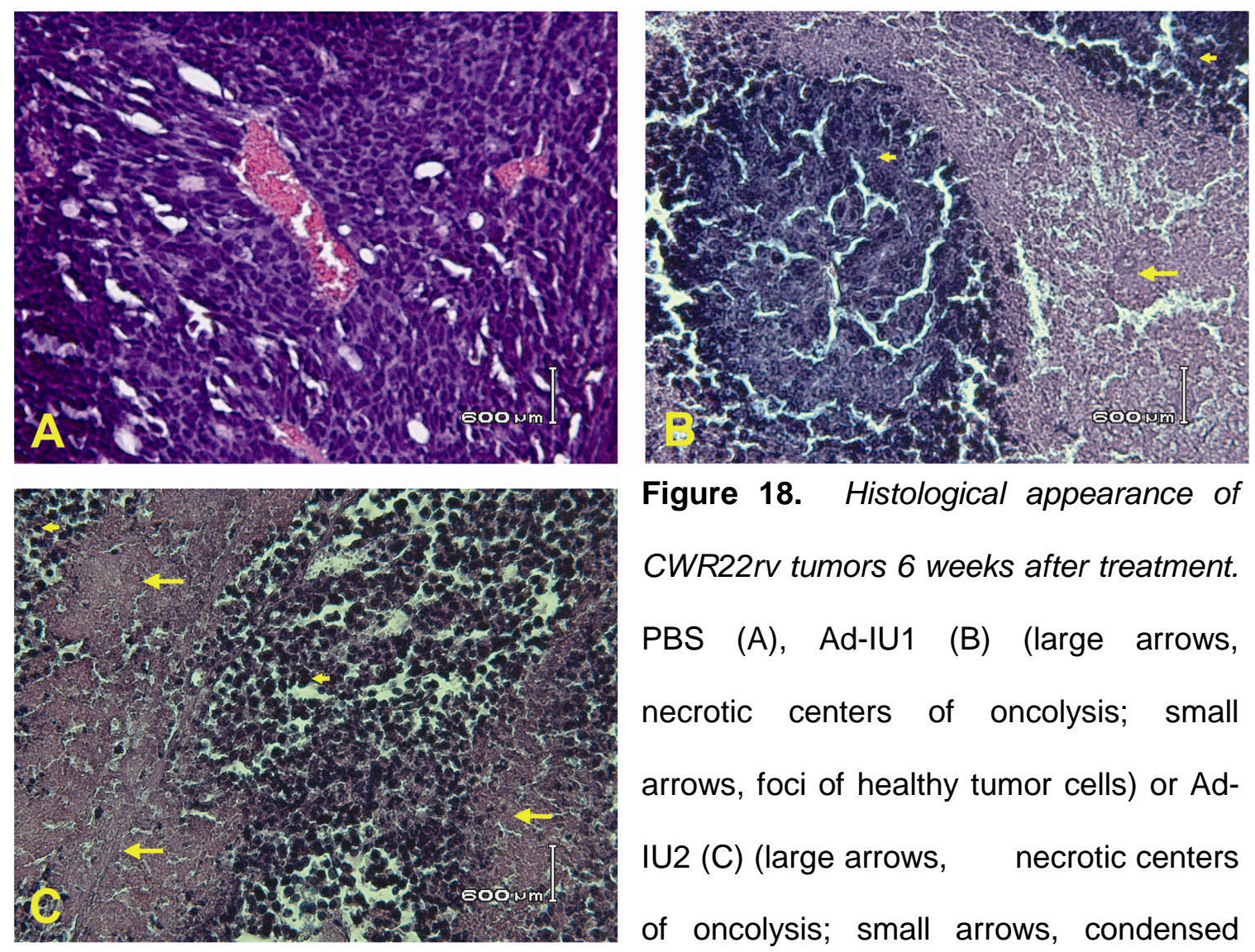

Figure 18. Histological appearance of CWR22rv tumors 6 weeks after treatment. PBS (A), Ad-IU1 (B) (large arrows, necrotic centers of oncolysis; small arrows, foci of healthy tumor cells) or AdIU2 (C) (large arrows, necrotic centers of oncolysis; small arrows, condensed nuclei) (H\&E, 200X). Treatment effect of Ad-IU2 was diffuse throughout a greater extent of the tumor, unlike the focal appearance of Ad-IU1, suggesting a TRAIL-mediated bystander effect. 

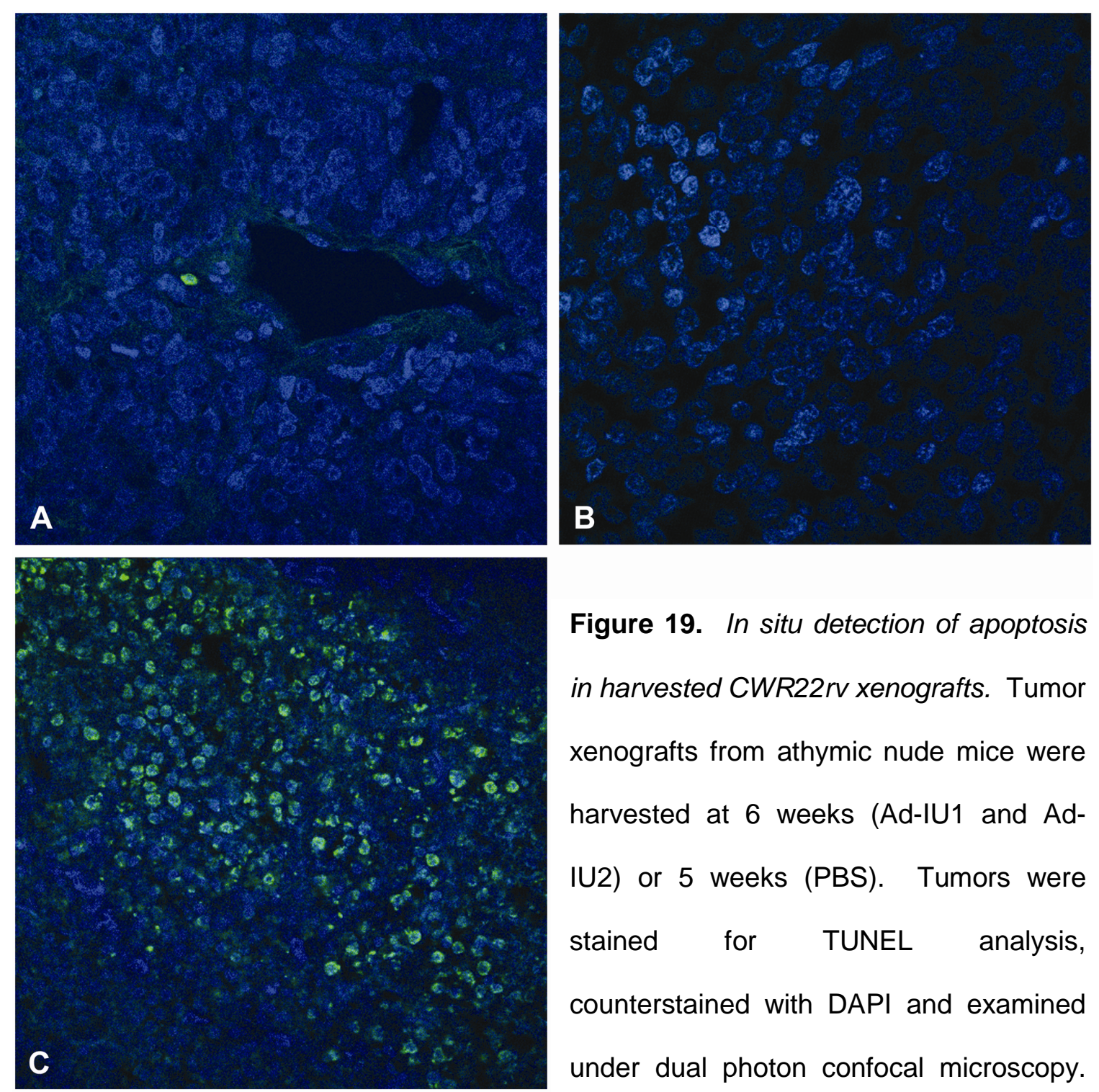

Figure 19. In situ detection of apoptosis in harvested CWR22rv xenografts. Tumor xenografts from athymic nude mice were harvested at 6 weeks (Ad-IU1 and AdIU2) or 5 weeks (PBS). Tumors were stained for TUNEL analysis, counterstained with DAPI and examined under dual photon confocal microscopy.

No significant apoptosis was detected in tumors treated with PBS (A) or Ad-IU1 (B) and marked apoptosis was detected in tumors treated with Ad-IU2 (C). 


\section{Radio-Sensitization of Androgen-Independent Prostate Cancer with Ad-IU2}

\section{Background}

Recent studies supporting the use of EBRT as standard of care for high risk prostate cancer have also demonstrated that monotherapy is insufficient for the treatment of locally advanced disease, leading to higher rates of biochemical failure and PCSM (39, 280-282). Furthermore, clinical studies indicate that adequate locoregional elimination of prostate cancer is necessary to decrease the risk of metastases from a nidus of continuously shedding cancer cells (50). Recently, this has been achieved by improved targeting and dose escalation of EBRT through the use of 3D-CRT and IMRT $(283,284)$. However, the persistence of radio-resistant prostate cancer cells and doselimiting toxicities to surrounding normal tissues in the pelvis, such as the rectum and bladder, prevent complete tumor eradication, leading to failure of the therapy and a potentially fatal disseminated disease. For these reasons, the development of novel therapies to enhance the efficacy of conventional treatments is critically needed.

Recently, the combination of EBRT and chemotherapy has been evaluated clinically to achieve improved locoregional control of a tumor following radiotherapy (285). Although the greatest clinical efficacy has been achieved for head and neck (286), non-small cell lung (287), and cervical (288) cancer, chemoradiotherapy with docetaxel is currently being tested in early clinical trials for prostate cancer $(289,290)$. Radiation sensitizers, such as chemotherapy, modulate the response of cancer cells to EBRT leading to improved local tumor control and through dose-reduction, protection of normal tissue from the acute toxicity of radiation. The ideal radiosensitizer would have adequate spread throughout the entire tumor, sensitize tumor cells selectively and cause minimal toxicity to normal tissue. 
Over the past fifteen years, gene therapy has been widely studied as an alternative approach to the treatment of prostate cancer; however, current limitations of viral replication and transduction efficiency reduce its clinical feasibility as a monotherapy. Likewise, the efficacy of EBRT for prostate cancer is limited by toxicity to the surrounding organs. In the first half of my thesis, I evaluated the preclinical efficacy of Ad-IU2 as a single modality against androgen-independent prostate cancer. In addition to being a powerful tumor-specific cytotoxic molecule, TRAIL has proven to be a potent radio-sensitizing agent. I hypothesized that the neoadjuvant treatment of high risk prostate cancer with a PSRCA expressing TRAIL would result in the radiosensitization of tumor cells, thereby enhancing the therapeutic effect of EBRT. The goals of this multi-modal approach combining viral lysis, apoptosis-inducing gene therapy, and radiation therapy are to achieve complete local tumor control, reduce radiation dose and associated treatment morbidities, and improve the clinical outcome for patients with high risk locally advanced prostate cancer.

Recent investigations have demonstrated the potentiation of the antitumor activity of EBRT by combining it with TRAIL. Initially, the link between TRAIL and RT was made in non-Hodgkin's patients undergoing EBRT, where TRAIL expression was upregulated in blood cells distant from the irradiated tissue. This was thought to provide some therapeutic benefit and contribute partially to the abscopal effect of RT $(291,292)$. To further support the role of rhTRAIL in radiation-induced apoptosis, DR5-knockout mice exhibited reduced amounts of apoptosis in tissues exposed to ionizing radiation (293). Pretreatment of cancer cells with soluble TRAIL or an adenoviral vector expressing TRAIL resulted in sensitization to EBRT. In DU-145 prostate cancer cells, a synergistic effect on apoptosis was observed with combinatorial therapy and was found to be Bax-dependent, implicating a role for the mitochondrial or intrinsic apoptosisamplification loop (294). El Hassan et al. also described the role of the intrinsic 
mitochondrial pathway in the response to combined treatment. In non-small cell lung cancer cell lines, synergy was accompanied by caspase-8 and Bid cleavage, which was reversible by Bcl-2 overexpression (295). On the other hand, the mechanism of synergy in Jurkat cells did not appear to be related to death receptor upregulation or mitochondrial apoptosis, as cytochrome c was not released from the mitochondrial membrane and $\mathrm{Bcl}-2$ overexpression as well as p53 mutation did not inhibit apoptosis (296). This multimodal approach has been studied preclinically for three types of cancer. An RGD-retargeted adenovirus, Ad/TRAIL-F/RGD, expressing full-length TRAIL under control of an hTERT/GAL4VP16/GT promoter system, has been investigated for use in esophageal adenocarcinoma, where combination with EBRT resulted in enhanced apoptosis induction and decreased clonogenic survival. This radio-sensitization appeared to be synergistic and specific to cancer cells. Furthermore, combined therapy inhibited tumor growth in vivo and prolonged nude mouse survival from 16.7 and 21.5 days (RT and Ad/TRAIL-F/RGD alone, respectively) to 31.6 days (combined) in the esophageal adenocarcinoma model (297). Similarly, Ad/TRAIL-F/RGD improved nude mouse survival from 16.5 and 23.7 days (RT and Ad/TRAIL-F/RGD alone, respectively) to 43.7 days (combined) in a non-small cell lung cancer model (298). Kaliberov et al. developed a replication-deficient adenovirus, AdFIt-TRAIL, which expressed full-length TRAIL under control of the VEGF receptor promoter, FLT-1. AdFIt-TRAIL was studied for the treatment of prostate cancer in DU-145 cells where cell death in combination with RT was merely additive. In an athymic mouse DU-145 xenograft model, there was no significant difference between tumors treated with AdFIt-TRAIL alone or in combination with RT (299). The previous study was limited by the use of a replication-deficient virus and the weak activity of Flt-1 promoter in DU-145 cells. In the current study, I improved upon this approach through the use of a replication-competent adenovirus in which 
replication and TRAIL expression are driven by a prostate-specific promoter with strong activity in PSA/PSMA-positive prostate cancer cells.

\section{Evaluation of the Combination of Ad-IU2 and Radiation Therapy for the Treatment of Androgen-Independent Prostate Cancer \\ Ad-IU2 Reduced the Clonogenic Survival of Irradiated Prostate Cancer Cells More Effectively Than rhTRAIL Alone}

To test the combined effect of TRAIL gene therapy with Ad-IU2 and radiotherapy, I first determined the optimal dose of virus required to produce the greatest radiosensitization of prostate cancer cells. A radiosensitizing agent should decrease the clonogenic survival of cancer cells following irradiation when administered in sub-optimal concentrations for use as a single agent. For this reason, in accordance with data from the in vitro cytotoxicity assay (Figure 11), the dose range of 0.0015 to 0.0045 LDU/cell was selected. CWR22rv cells were pretreated with the above concentrations of Ad-IU2 24 hours prior to irradiation and then allowed to rest for 24 hours prior to plating for

clonogenicity. 3 weeks after plating, colonies were counted and survival data fitted to the linear-quadratic regression model, normalizing for viral cytotoxicity with each RT dose. Radiosensitization of CWR22rv cells following infection with Ad-IU2 was evidenced by a reduction in the shoulder of the clonogenic survival curve (Figure 20). The Ad-IU2 dose of $0.0015 \mathrm{LDU} /$ cell resulted in the least reduction of clonogenic survival, while an Ad-IU2 dose of 0.003 LDU/cell produced the greatest radiosensitization. Interestingly, this effect was not strictly dose-dependent, as a higher dose of 0.0045 LDU/cell of Ad-IU2 resulted in less reduction of survival fraction. As a result, the viral dose 0.003 LDU/cell was chosen for use in subsequent combinatorial experiments. 


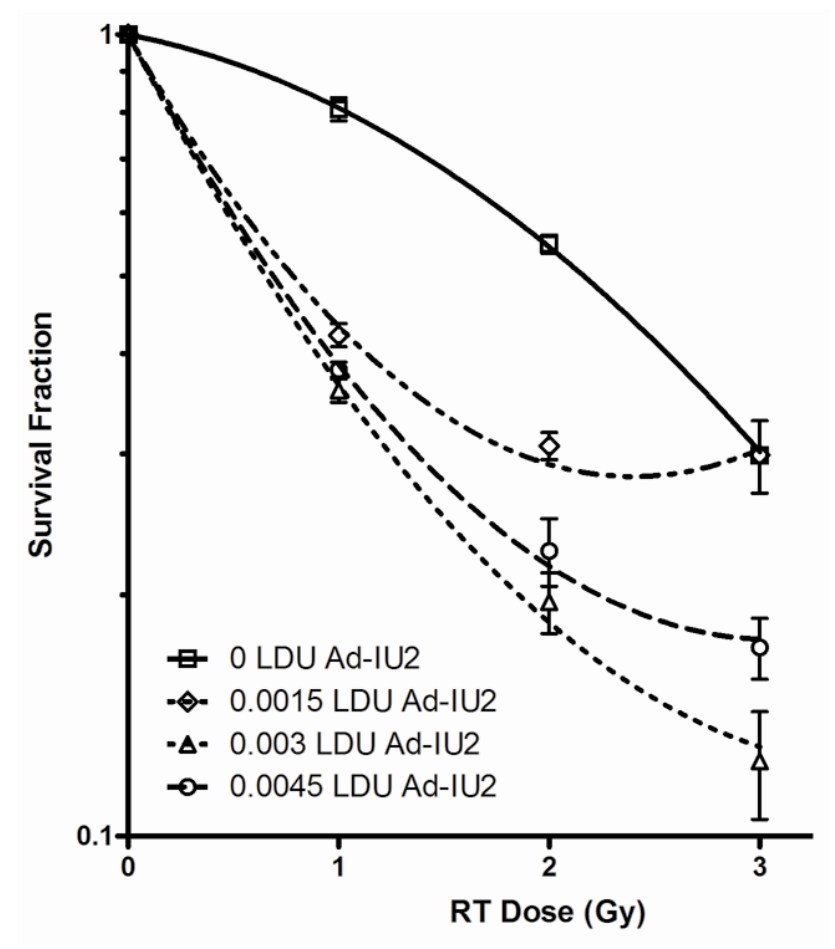

Figure 20. Determination of optimal viral dose for clonogenic assay. $1 \mathrm{x}$ $10^{6}$ CWR22rv cells were infected with $0,0.0015,0.003$ or 0.0045 LDU/cell Ad-IU2 for 24 hours and irradiated with $0,1,2$ or 3 Gy. 24 hours after irradiation, cells were plated at low densities in p100 dishes. 3 weeks after seeding, plates were stained with crystal violet, and colonies of 50 cells or greater were counted. The

dose response survival curve was fitted to the linear-quadratic regression model [ In $\left.\left(S F / S F_{0}\right)=-\left(\alpha D+\beta D^{2}\right)\right] .0 .003$ LDU/cell Ad-IU2 was chosen as the optimal dose for future experiments.

The treatment of CWR22rv cells with ionizing radiation resulted in a dosedependent decrease in clonogenicity, exhibiting a broad shoulder in the radiation survival curve (Figure 21). Pretreatment of CWR22rv cells with 0.003 LDU/cell AdE4PSESE1a, PSRCA vector control, resulted in a minimal left-ward shift in the survival curve and no reduction of the shoulder, indicating no radio-sensitization by a PSRCA alone. On the other hand, pretreatment of the PSA/PSMA-positive cells with 0.003 LDU/cell Ad-IU2 resulted in a dramatic reduction of clonogenic survival, indicating radiosensitization. Pretreatment of cells with rhTRAIL $(75 \mathrm{ng} / \mathrm{ml})$ resulted in a decrease in clonogenic survival of irradiated CWR22rv cells and a reduction in the shoulder of the radiation survival curve; however, radio-sensitization by rhTRAIL alone was not as 
dramatic as that achieved by Ad-IU2, despite similar concentrations of TRAIL protein in both treatment groups (Figure 15). These data suggest that the expression of full-length TRAIL by a replication-competent vector is a more potent radio-sensitizer than the administration of rhTRAIL.

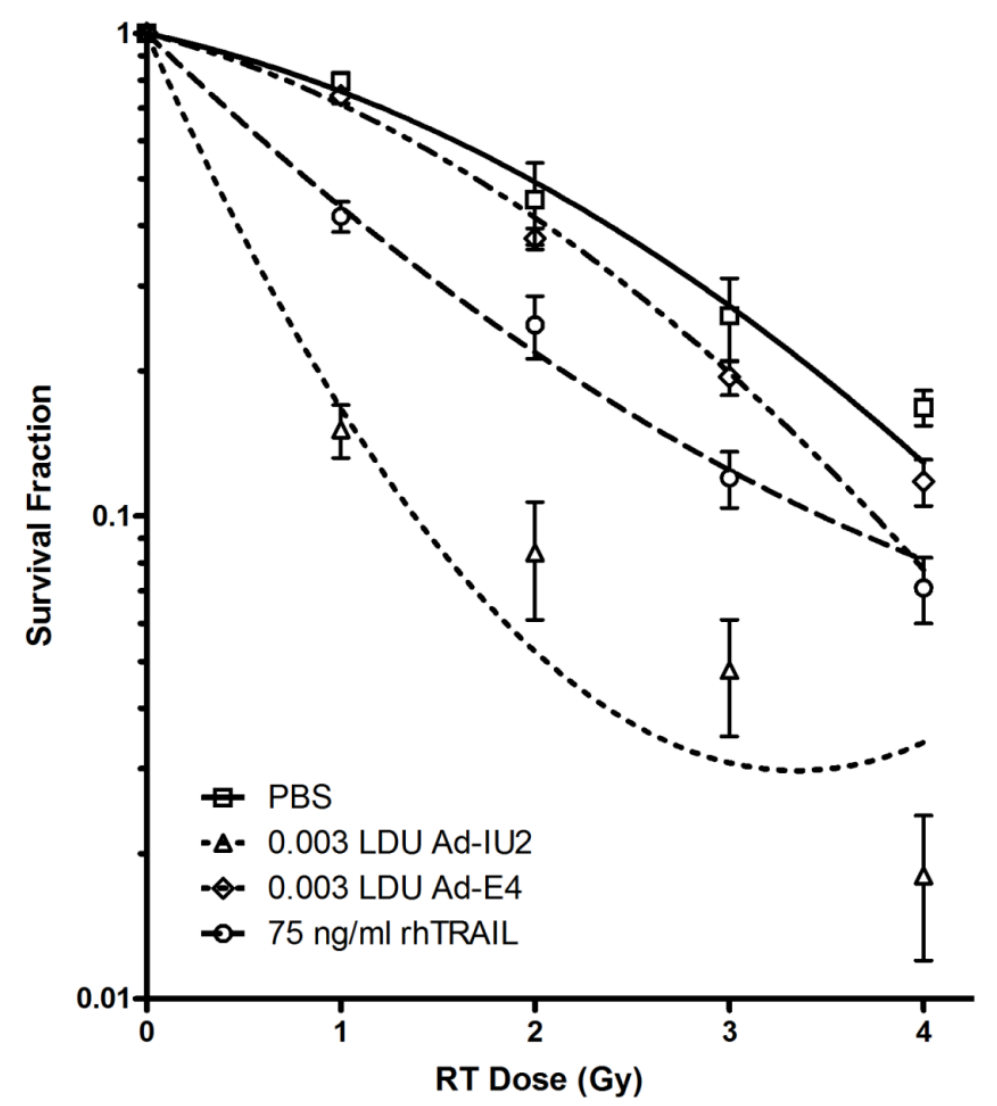

Figure 21. Combination of Ad-IU2 and RT resulted in a decrease in the clonogenicity of CWR22rv cells. $1 \times 10^{6}$ CWR22rv cells were plated overnight in a T24 flask and treated with $0.003 \mathrm{LDU} / \mathrm{cell}$ Ad-IU2 or Ad-E4, rhTRAIL (75 $\mathrm{ng} / \mathrm{ml}$ ) or PBS. 24 hours post-treatment, cells were irradiated at a dose of 0,1 , 2, 3 or 4 Gy and plated at

low densities in p100 dishes 24 hours after irradiation. 3 weeks after seeding, plates were stained with crystal violet, and colonies of 50 cells or greater were counted. The dose-response survival curve was fitted to the linear-quadratic regression model [ In $\left.\left(S F / S F_{0}\right)=-\left(\alpha D+\beta D^{2}\right)\right]$. Radio-sensitization was evidenced by a reduction in the shoulder of the curves for cells treated with rhTRAIL and Ad-IU2 and was greatest for cells infected with Ad-IU2. 
Compared to PBS or Ad-E4PSESE1a, Ad-IU2 drastically reduced the clonogenic survival of CWR22rv cells. EBRT at a dose of 2 Gy resulted in a survival fraction of $49.2 \%$ following monotherapy or $41.5 \%$ in combination with Ad-E4PSESE1a; however, in cells pretreated with Ad-IU2, irradiation with 2 Gy resulted in a survival fraction of $5.24 \%$, compared to $21.8 \%$ for cells pretreated with rhTRAIL. The mean lethal dose of ionizing radiation was reduced from 2.52 Gy for mock-treated cells to 0.512 Gy for CWR22rv cells pretreated with Ad-IU2. This radio-sensitization corresponded to dose reduction factors (DRF) of 4.91 for $0.003 \mathrm{LDU} / \mathrm{cell}$ Ad-IU2 and 2.06 for $75 \mathrm{ng} / \mathrm{ml}$ rhTRAIL (Table 5).

Table 5. Ad-IU2 sensitized CWR22rv cells to EBRT. The linear-quadratic regression analysis for CWR22rv cells demonstrated radio-sensitization as evidenced by an increase in the lethal, irreparable events $(\alpha)$, a reduction in sublethal damage $(\beta)$ component, and a reduction in the $\mathrm{D}_{0}$ (RT dose at which $37 \%$ of cells for colonies) from 2.52 Gy with RT alone to 0.0524 Gy in combination with $0.003 \mathrm{LDU} / \mathrm{cell} \mathrm{Ad}-\mathrm{IU} 2$, resulting in a dose reduction factor (DRF) of 4.91 for Ad-IU2. Radio-sensitization was specific to Ad-IU2, as infection with $0.003 \mathrm{LDU} / \mathrm{cell}$ Ad-E4PSESE1a failed to enhance the radiation effect. Greater radio-sensitization was achieved with Ad-IU2 than with $75 \mathrm{ng} / \mathrm{ml}$ rhTRAIL which had a DRF of 2.06. $R^{2}$, regression coefficient; SF2, surviving fraction at 2 Gy.

\begin{tabular}{|c|cccc|}
\cline { 2 - 5 } \multicolumn{1}{c|}{} & PBS & Ad-IU2 & Ad-E4 & rhTRAIL \\
\hline $\mathbf{R}^{2}$ & 0.990 & 0.997 & 0.993 & 0.998 \\
$\boldsymbol{\alpha}$ & 0.198 & 2.10 & 0.240 & 0.894 \\
$\boldsymbol{\beta}$ & 0.0786 & -0.314 & 0.100 & -0.0666 \\
$\mathbf{\alpha} / \boldsymbol{\beta}$ & 2.51 & -6.69 & 2.39 & -13.4 \\
$\mathbf{S F 2}$ & 0.492 & 0.0524 & 0.415 & 0.218 \\
$\mathbf{D}_{0}$ & $2.52 \mathrm{~Gy}$ & $0.512 \mathrm{~Gy}$ & $2.18 \mathrm{~Gy}$ & $1.22 \mathrm{~Gy}$ \\
$\mathbf{D R F}$ & & 4.91 & 1.16 & 2.06 \\
\hline
\end{tabular}


Compared to CWR22rv cells, C4-2 cells were more radio-sensitive, as indicated by a steeper slope to the radiation dose-response curve and a narrower shoulder to the curve (Figure 22). Likewise, the mean lethal dose for C4-2 cells was 2.35 Gy, compared

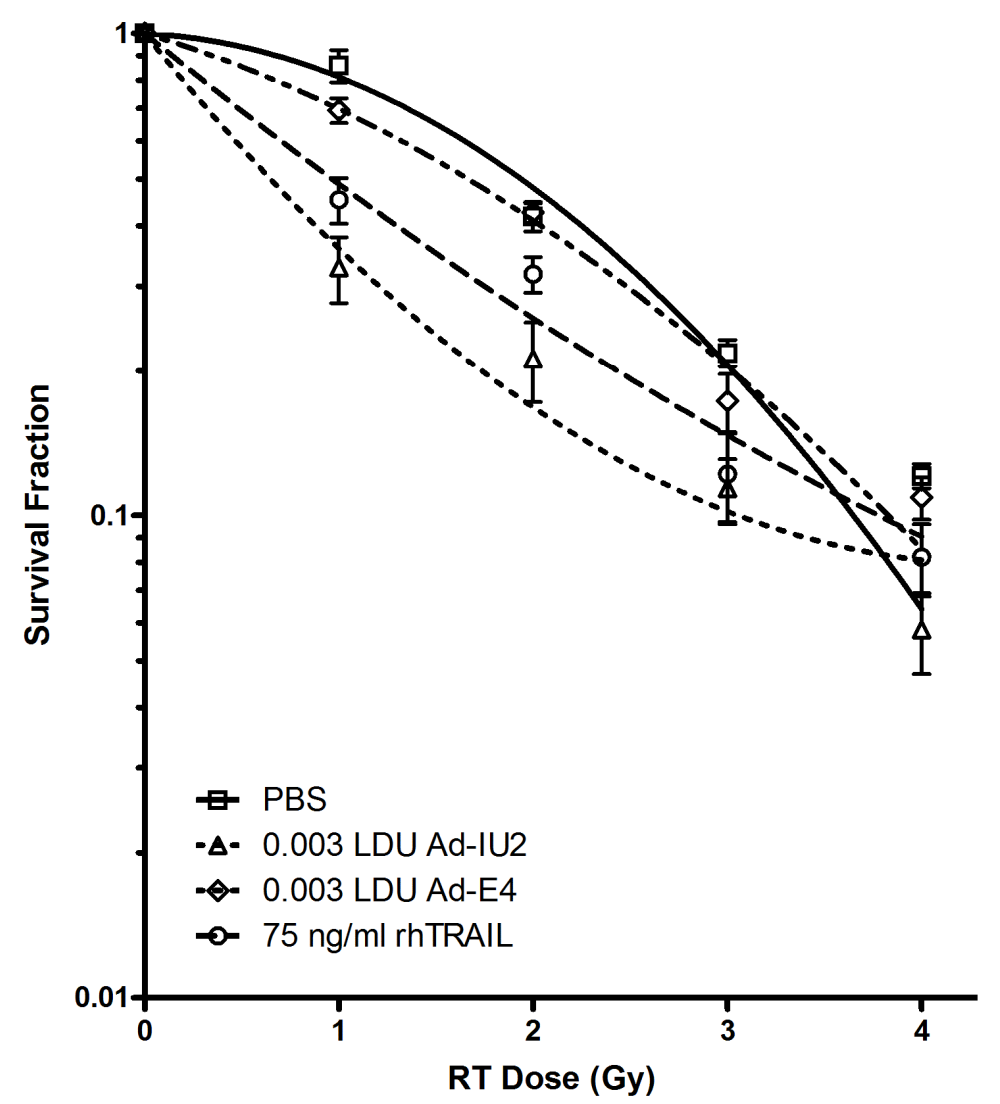

Figure 22. Combination of Ad-IU2 and $R T$ resulted in a decrease in the clonogenicity of C4-2 cells. $1 \times 10^{6}$ C4-2 cells were plated overnight in a T24 flask and treated with 0.003 LDU/cell Ad-IU2 or Ad-E4, rhTRAIL (75 ng/ml) or PBS. 24 hours post-treatment, cells were irradiated at a dose of $0,1,2,3$ or 4 Gy and plated at low densities

in p100 dishes 24 hours after irradiation. 2 weeks after seeding, plates were stained with crystal violet, and colonies of 50 cells or greater were counted. The dose-response survival curve was fitted to the linear-quadratic regression model. Radio-sensitization was evidenced by a reduction in the shoulder of the curves for cells treated with rhTRAIL and Ad-IU2 and was greatest for cells infected with Ad-IU2.

to 2.52 Gy for CWR22rv cells (Table 6). The treatment effect was less dramatic in C4-2 cells than in CWR22rv cells; however, pretreatment of C4-2 cells with both rhTRAIL and 
Table 6. Ad-IU2 sensitized C4-2 cells to EBRT. The linear-quadratic regression model [ In $\left.(S F / S F 0)=-\left(\alpha D+\beta D^{2}\right)\right]$ for $C 4-2$ cells demonstrated radio-sensitization as evidenced by an increase in the $\alpha$ component, a reduction in the $\beta$ component, and a reduction in the $\mathrm{D}_{0}$ (RT dose at which $37 \%$ of cells for colonies) from 2.35 Gy with RT alone to 0.168 Gy in combination with 0.003 LDU/cell Ad-IU2, This resulted in a dose reduction factor (DRF) of 2.35 for Ad-IU2. Radio-sensitization was specific to Ad-IU2, as infection with 0.003 LDU/cell Ad-E4PSESE1a failed to enhance the radiation effect. Greater radiosensitization was achieved with Ad-IU2 than with $75 \mathrm{ng} / \mathrm{ml}$ rhTRAIL which had a DRF of 1.66. $R^{2}$, regression coefficient; SF2, surviving fraction at 2 Gy.

\begin{tabular}{|c|cccc|}
\cline { 2 - 5 } \multicolumn{1}{c|}{} & PBS & Ad-IU2 & Ad-E4 & rhTRAIL \\
\hline $\mathbf{R}^{2}$ & 0.985 & 0.994 & 0.997 & 0.990 \\
$\boldsymbol{\alpha}$ & 0.0476 & 1.16 & 0.275 & 0.756 \\
$\boldsymbol{\beta}$ & 0.160 & -0.132 & 0.0852 & -0.0389 \\
$\boldsymbol{\alpha} / \boldsymbol{\beta}$ & 0.298 & -8.76 & 3.22 & -19.4 \\
$\mathbf{S F 2}$ & 0.480 & 0.168 & 0.411 & 0.257 \\
$\mathbf{D}_{0}$ & $2.35 \mathrm{~Gy}$ & $0.966 \mathrm{~Gy}$ & $2.17 \mathrm{~Gy}$ & $1.42 \mathrm{~Gy}$ \\
$\mathbf{D R F}$ & & 2.43 & 1.08 & 1.66 \\
\hline
\end{tabular}

Ad-IU2 markedly decreased the colony formation of irradiated cells and reduced the shoulder of the curve. In C4-2 cells, 2 Gy of radiation alone decreased clonogenic survival by $52 \%$, whereas the addition of $75 \mathrm{ng} / \mathrm{ml}$ rhTRAIL or 0.003 LDU/cell Ad-IU2 reduced survival by 74.3 and $83.2 \%$, respectively. The resulting DRFs were 2.43 for AdIU2 and 1.66 for rhTRAIL. These results suggest that TRAIL gene therapy with Ad-IU2 is a more potent radiation-sensitizing agent than rhTRAIL in PSA/PSMA-positive androgen-independent prostate cancer cell lines and that reduction of EBRT dose can be achieved by pretreatment with Ad-IU2. 


\section{Radio-Sensitization by Ad-IU2 was Specific to PSA/PSMA-Positive Prostate Cancer Cells}

Due to the proximity of the prostate to the rectum and the bladder, I tested whether Ad-IU2 would sensitize cells from the surrounding normal tissues. For this reason, the colorectal adenocarcinoma cell line, LoVo, and bladder transitional cell carcinoma cell line, T24, were selected. Infectivity assays were performed to achieve equal infectivity of Ad5 in these cell lines as in prostate cancer cell lines. LoVo and T24 cells were infected with Ad5-GFP and analyzed by FACS analysis for GFP expression. As depicted in Figure 23, 500 vp/cell Ad5-GFP caused a shift in GFP expression of $14.68 \%$ in LoVo cells and $10,000 \mathrm{vp} /$ cell caused a shift of $11.47 \%$ in T24 cells. To
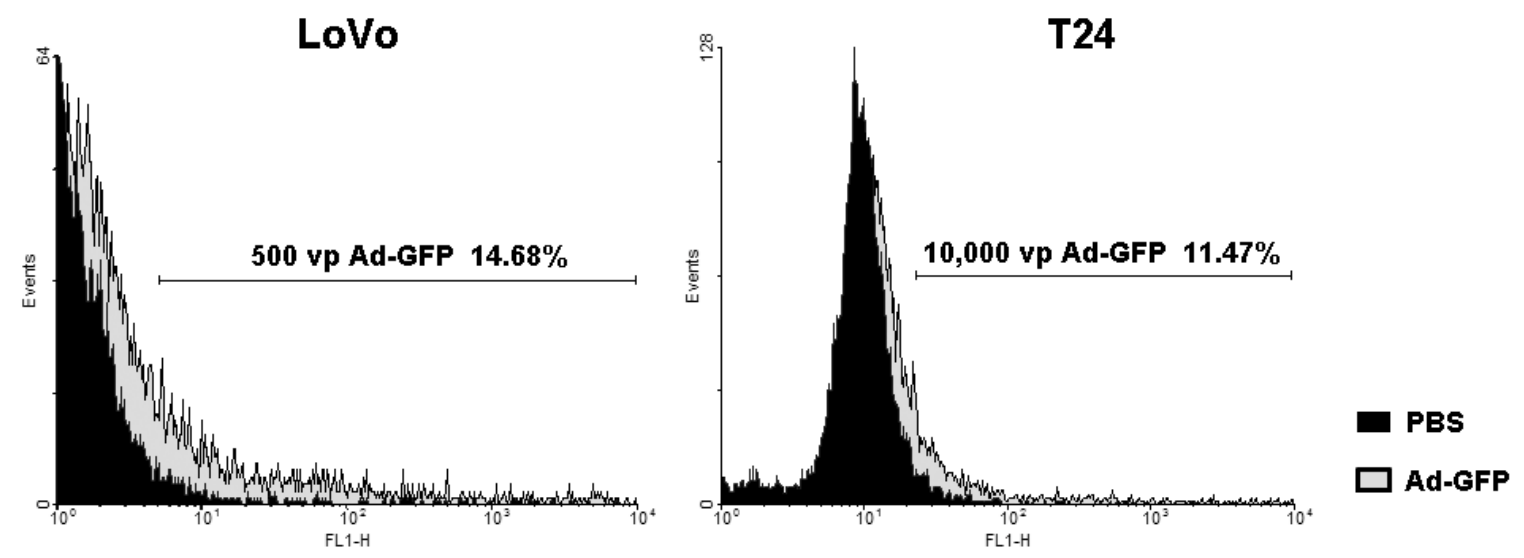

Figure 23. Adenoviral infectivity assay for LoVo and T24 cells. LoVo and T24 cells were infected with varying doses of Ad5-GFP, a replication-defective adenovirus expressing GFP under control of the CMV promoter. For LoVo cells, a range of 0 to $5,000 \mathrm{vp} / \mathrm{cell}$ was used, and for T24 cells, a range of 0 to 10,000 vp/cell was used. Twenty-four hours after infection, GFP expression was analyzed by FACS analysis.

achieve equal infectivity, LoVo required 57 -fold more adenoviral vector than CWR22rv cells, while T24 cells required 1400-times the adenoviral dose (Table 7). 
Table 7. Relative infectivity of LoVo and T24 cells for Ad5. CWR22rv, LoVo and T24 cells were infected with varying doses of Ad5-GFP for 24 hours and analyzed for GFPpositivity (\% FL1-H) by FACS analysis. Values for LoVo and T24 cells were compared to CWR22rv values to determine relative infectivities, which were used in subsequent experiments to standardize viral doses.

\begin{tabular}{|c|c|c|c|c|}
\hline & $\begin{array}{l}\text { Ad5-GFP } \\
\text { (vp/cell) }\end{array}$ & $\%$ FL1-H & CWR22rv & Rel. Infectivity \\
\hline $\begin{array}{l}\text { LoVo } \\
\text { T24 }\end{array}$ & $\begin{array}{c}500 \\
10,000\end{array}$ & $\begin{array}{l}14.68 \\
11.47\end{array}$ & $\begin{array}{l}8.70 \mathrm{vp} / \mathrm{cell} \\
6.85 \mathrm{vp} / \mathrm{cell}\end{array}$ & $\begin{array}{c}57 X \\
1,460 X\end{array}$ \\
\hline
\end{tabular}

To determine whether combinatorial treatment sensitized colorectal or bladder cancer cell lines to radiotherapy, LoVo and T24 cells were pretreated with cell linespecific equivalent doses of Ad-IU2 or Ad-E4PSESE1a for 24 hours and then irradiated. Because distinct colony formation was difficult to achieve in LoVo and T24 cells, cell viability was assessed by MTT assay, rather than clonogenic assay. As depicted in Figure 24, treatment of LoVo and T24 cells with EBRT resulted in a dose-dependent decrease in cell viability. LoVo cells demonstrated a higher basal level of radiosensitivity than T24 cells. Pretreatment of both PSA/PSMA-negative cell lines with AdIU2 did not enhance cell killing. This was expected, as TRAIL was under transcriptional control of PSES; however, the effect of rhTRAIL on LoVo and T24 cells was not evaluated due to its lack of tissue-specificity.

\section{Combinatorial Therapy Failed to Augment Induction of Apoptosis}

Previous studies have demonstrated that the enhanced cell killing and radiosensitization that occurred from the combination of TRAIL and EBRT was caused by enhanced apoptosis induction; however, most studies used high doses of radiation 
LoVo

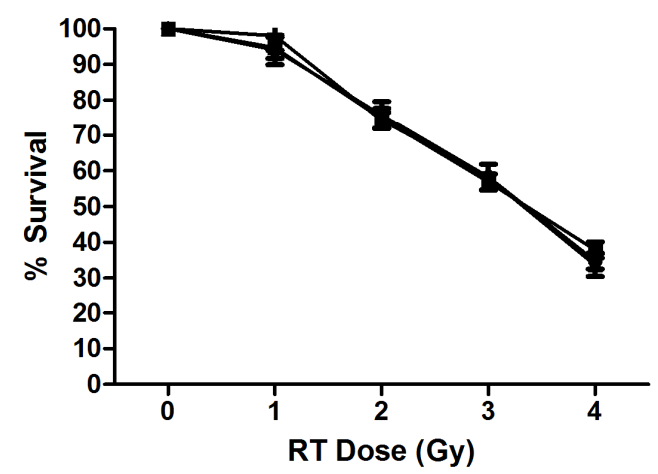

T24

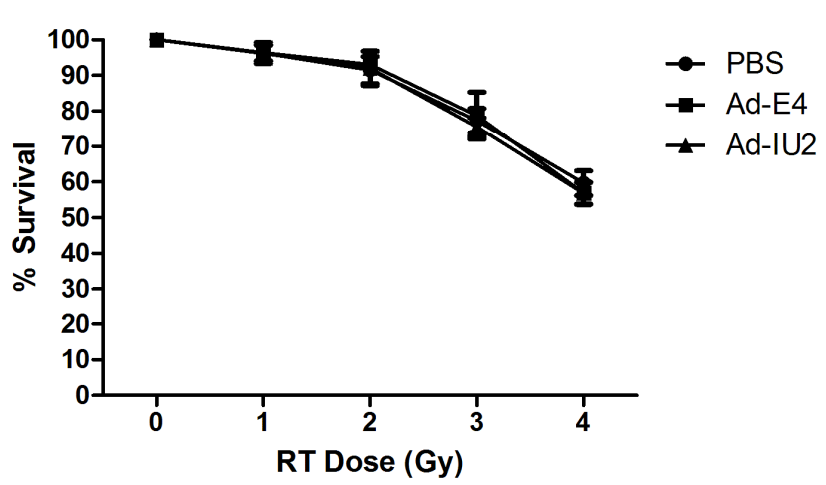

Figure 24. Ad-IU2 failed to sensitize PSA/PSMA-negative colorectal and bladder cancer cell lines to EBRT. LoVo and T24 cells were plated in 96-well plates and infected with Ad-IU2 or Ad-E4PSESE1a in doses adjusted for relative infectivity of each cell line. Twenty-four hours after infection, cells were irradiated with 0, 1, 2, 3 or 4 Gy. Cells were analyzed for survival by MTT assay at 96 hours post-irradiation for LoVo cells or 48 hours post-irradiation for T24 cells. Ad-IU2 failed to enhance the sensitivity of PSA/PSMA-negative cell lines to EBRT.

ranging from 5 to 40 Gy $(294,295,299)$. To determine whether the enhanced cell killing of androgen-independent prostate cancer cell lines was due to increased apoptosis with the lower RT doses and suboptimal viral doses used in this study, I analyzed Annexin V and PI staining of cells treated with Ad-IU2 and EBRT. As seen in Figure 25, in both CWR22rv and C4-2 cells, infection with 0.003 LDU/cell Ad-IU2 resulted in a slight induction of apoptosis above that of the PSRCA control. Treatment of CWR22rv and C4-2 cells with 3 Gy RT resulted in nearly 2 -fold induction of apoptosis above that of mock PBS-treated cells; however, no increase in apoptosis induction was observed in the combined treatment group. These data suggest that at the minimal doses of virus and EBRT required to reduce clonogenic survival in CWR22rv and C4-2 cells, 
augmentation of apoptosis induction did not contribute to the mechanism of radiosensitization.
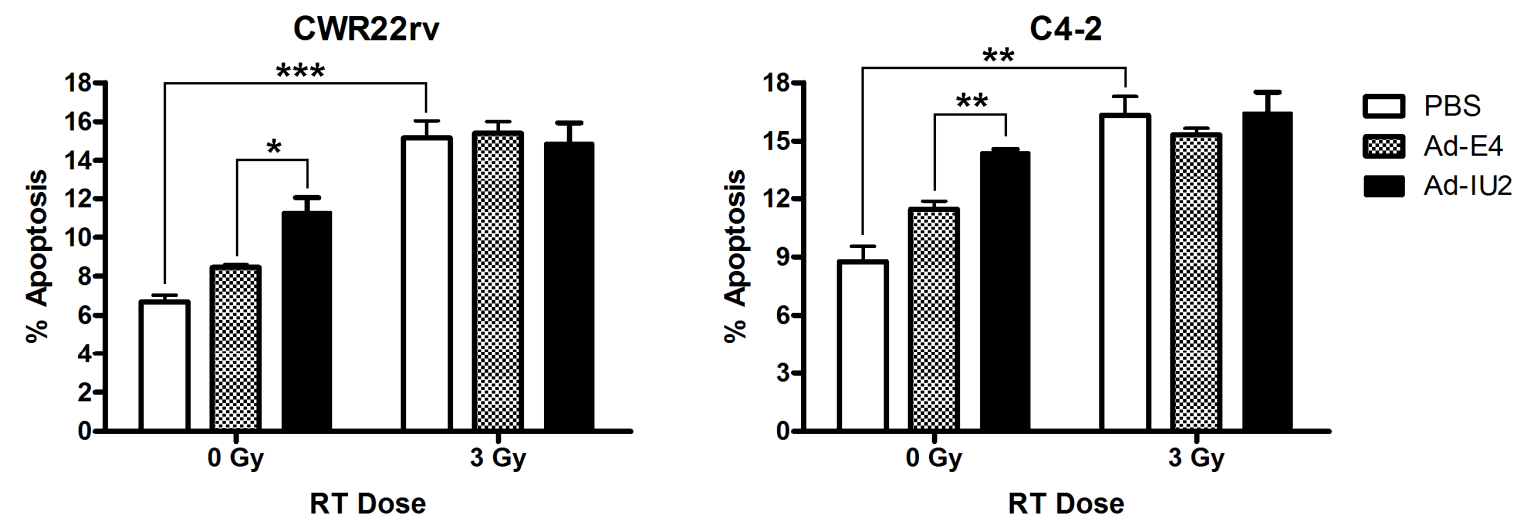

Figure 25. Combination of Ad-IU2 plus EBRT did not enhance apoptosis induction at doses sufficient to decrease clonogenicity of PSA/PSMA-positive prostate cancer cells. CWR22rv and C4-2 cells were treated with 0.003 LDU/cell Ad-IU2, Ad-E4PSESE1a or PBS and irradiated 24 hours after infection for a dose of 0 or 3 Gy. Twenty-four hours after irradiation, cells were harvested and analyzed for apoptosis by Annexin V-FITC and PI staining. At a low dose of 0.003 LDU/cell, Ad-IU2 significantly induced apoptosis compared to PSRCA control alone; however, this level was lower than that induced by 0.01 LDU/cell (Figure 9), as expected. In addition, EBRT at a dose of 3 Gy was sufficient to induce significant levels of apoptosis compared to untreated cells; however, in combination, $0.003 \mathrm{LDU} /$ cell Ad-IU2 failed to augment RT-induced apoptosis induction. ${ }^{*}=p<0.05,{ }^{* *}=p<0.01,{ }^{\star * *}=p<0.001$. 


\section{Pretreatment of Androgen-Independent Prostate Cancer Cells with Ad-IU2 or rhTRAIL Resulted in $G_{1}$ Phase Accumulation and Diminished Radiation-Induced $G_{2}$ Phase Arrest}

To elucidate the mechanism for radio-sensitization of prostate cancer cells by Ad-IU2, I performed cell cycle analysis on the cells following pretreatment with Ad-IU2 or rhTRAIL and also following combination treatment. As depicted in Figure 26, infection of
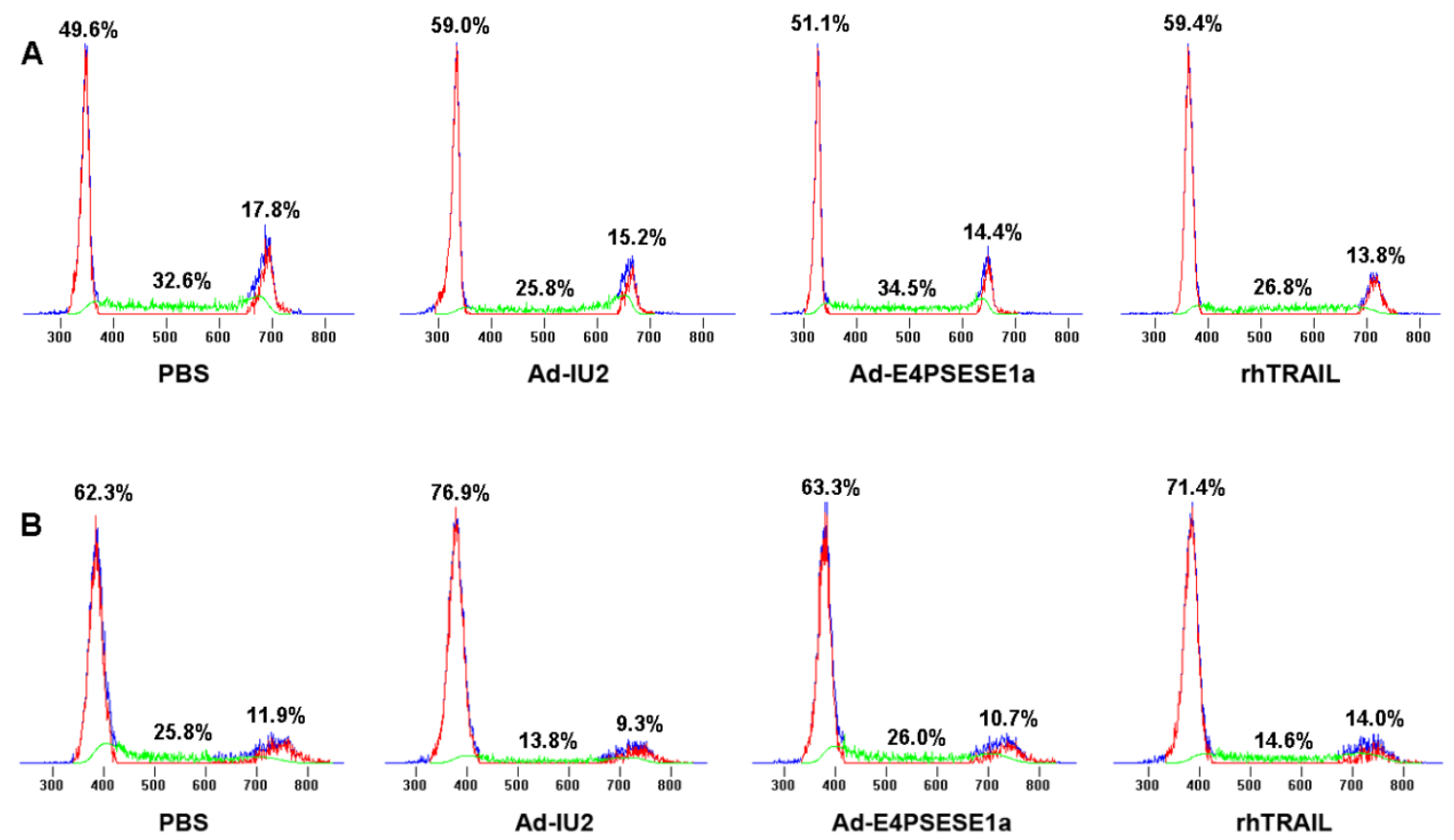

Figure 26. Treatment of CWR22rv and C4-2 cells with Ad-IU2 or rhTRAIL resulted in accumulation of cells in $G_{1}$ phase. Androgen-independent prostate cancer cell lines CWR22rv (A) and C4-2 (B) were treated with PBS, $100 \mathrm{ng} / \mathrm{ml}$ rhTRAIL, $0.003 \mathrm{LDU} / \mathrm{cell}$ Ad-IU2 or Ad-E4PSESE1a for 24 hours. Cells were washed, harvested, permeabilized and treated with RNaseA. DNA content was stained with PI and analyzed by FACS analysis. \% cells in each phase of the cell cycle is listed for each histogram. Following treatment with Ad-IU2 or rhTRAIL, both prostate cancer cell lines arrested in $\mathrm{G}_{1}$ phase. 
cells with Ad-E4PSESE1a resulted in no significant change in DNA content, compared to PBS-treated cells. On the other hand, infection of cells with 0.003 LDU/cell Ad-IU2 or treatment with $100 \mathrm{ng} / \mathrm{ml}$ rhTRAIL produced approximately a $20 \%$ increase in the $\mathrm{G}_{1}$ population. This was a significant finding, as previous studies have demonstrated that cells arrested in the $\mathrm{G}_{1}$ or $\mathrm{G}_{2}$ phases of the cell cycle are most sensitive to ionizing radiation (300). This was consistent with the observation that C4-2 cells, which were more sensitive to ionizing radiation, had a greater percentage of cells in $\mathrm{G}_{1}$ phase before treatment with Ad-IU2 or rhTRAIL.

To determine whether pretreatment of prostate cancer cells with Ad-IU2 or rhTRAIL affected the cell cycle of irradiated cells, DNA content of CWR22rv and C4-2 cells, pretreated for 24 hours, was analyzed 8, 16 and 24 hours after irradiation. As seen in Table 8, 3 Gy RT induced a $G_{2}$ phase arrest which lasted up to 16 hours in CWR22rv cells and 24 hours for $C 4-2$ cells. A similar $G_{2}$ phase arrest was observed in cells pretreated with 0.003 LDU/cell Ad-E4PSESE1a. The radiation-induced $\mathrm{G}_{2}$ arrest was dramatically inhibited in cells pretreated for 24 hours with Ad-IU2 or rhTRAIL. This effect lasted up to 8 hours in CWR22rv cells or 16 hours in C4-2 cells after irradiation. In addition, accumulation of cells in $\mathrm{G}_{1}$ phase was observed in both irradiated and unirradiated for up to 8 or 16 hours after RT in CWR22rv or C4-2 cells, respectively. Representative DNA content histograms for CWR22rv and C4-2 cells 8 hours after combinatorial treatment are displayed in Figure 27. Diminishment of the RT-induced G2 arrest was most dramatic in C4-2 cells, with an approximately 55\% reduction for C4-2 and $40 \%$ for CWR22rv cells. These data suggest that combinatorial therapy with Ad-IU2 or rhTRAIL plus EBRT caused significant cell cycle redistribution, which may contribute to the mechanism of radio-sensitization of prostate cancer cells. 
Table 8. Combination of Ad-IU2 or rhTRAIL and EBRT resulted in perturbation of RT-induced $\mathrm{G}_{2}$ arrest. CWR22rv and C4-2 cells were pretreated with 0.003 LDU/cell Ad-IU2, Ad-E4PSESE1a or $100 \mathrm{ng} / \mathrm{ml}$ rhTRAIL for $24 \mathrm{hrs}$, irradiated and analyzed.

\begin{tabular}{|c|c|c|c|c|c|c|c|c|c|}
\hline \multirow[b]{2}{*}{ CWR22rv } & \multicolumn{3}{|c|}{8 hours after RT } & \multicolumn{3}{|c|}{16 hours after $\mathrm{RT}$} & \multicolumn{3}{|c|}{24 hours after $\mathrm{RT}$} \\
\hline & $\mathbf{G}_{1}(\%)$ & S (\%) & $\mathrm{G}_{2}(\%)$ & $\mathrm{G}_{1}(\%)$ & $\mathrm{S}(\%)$ & $\mathbf{G}_{2}(\%)$ & $\mathrm{G}_{1}(\%)$ & $\mathbf{S}(\%)$ & $\mathrm{G}_{2}(\%)$ \\
\hline $\begin{array}{c}0 \text { Gy PBS } \\
0 \text { Gy Ad-IU2 } \\
0 \text { Gy Ad-E4 } \\
0 \text { Gy rhTRAIL }\end{array}$ & $\begin{array}{l}46.7 \\
54.0 \\
45.9 \\
55.8\end{array}$ & $\begin{array}{l}40.2 \\
32.7 \\
37.7 \\
29.9\end{array}$ & $\begin{array}{l}13.1 \\
13.3 \\
16.4 \\
14.3\end{array}$ & $\begin{array}{l}50.4 \\
56.8 \\
54.8 \\
53.5\end{array}$ & $\begin{array}{l}35.6 \\
29.3 \\
32.5 \\
34.4\end{array}$ & $\begin{array}{l}14.0 \\
13.9 \\
12.7 \\
12.1\end{array}$ & $\begin{array}{l}50.9 \\
57.9 \\
54.0 \\
56.5\end{array}$ & $\begin{array}{l}32.6 \\
23.7 \\
32.8 \\
25.0\end{array}$ & $\begin{array}{l}16.5 \\
18.4 \\
13.2 \\
18.5\end{array}$ \\
\hline $\begin{array}{c}3 \text { Gy PBS } \\
3 \text { Gy Ad-IU2 } \\
3 \text { Gy Ad-E4 } \\
3 \text { Gy rhTRAlL }\end{array}$ & $\begin{array}{l}29.4 \\
47.4 \\
31.1 \\
52.2\end{array}$ & $\begin{array}{l}42.5 \\
35.4 \\
39.5 \\
30.1\end{array}$ & $\begin{array}{l}28.1 \\
17.2 \\
29.4 \\
17.7\end{array}$ & $\begin{array}{l}51.2 \\
50.4 \\
48.6 \\
45.7\end{array}$ & $\begin{array}{l}25.0 \\
21.8 \\
22.6 \\
28.4\end{array}$ & $\begin{array}{l}23.8 \\
27.8 \\
28.8 \\
25.9\end{array}$ & $\begin{array}{l}52.9 \\
50.7 \\
52.9 \\
64.0\end{array}$ & $\begin{array}{l}27.0 \\
23.5 \\
26.9 \\
10.5\end{array}$ & $\begin{array}{l}20.1 \\
25.8 \\
20.2 \\
25.5\end{array}$ \\
\hline
\end{tabular}

\begin{tabular}{|c|c|c|c|c|c|c|c|c|c|}
\hline \multirow[b]{2}{*}{ C4-2 } & \multicolumn{3}{|c|}{8 hours after RT } & \multicolumn{3}{|c|}{16 hours after RT } & \multicolumn{3}{|c|}{24 hours after $\mathrm{RT}$} \\
\hline & $\mathrm{G}_{1}(\%)$ & $\mathbf{S}(\%)$ & $\mathrm{G}_{2}(\%)$ & $\mathrm{G}_{1}(\%)$ & S (\%) & $\mathrm{G}_{2}(\%)$ & $\mathrm{G}_{1}(\%)$ & $\mathrm{S}(\%)$ & $\mathrm{G}_{2}(\%)$ \\
\hline 0 Gy PBS & 64.5 & 28.7 & 6.8 & 72.2 & 23.9 & 3.9 & 71.0 & 20.9 & 8.1 \\
\hline 0 Gy Ad-IU2 & 79.7 & 13.7 & 6.6 & 86.6 & 10.3 & 3.1 & 80.0 & 8.3 & 11.7 \\
\hline 0 Gy Ad-E4 & 62.8 & 26.5 & 10.7 & 72.1 & 22.9 & 5.0 & 70.2 & 16.7 & 13.1 \\
\hline 0 Gy rhTRAIL & 74.5 & 17.4 & 8.1 & 86.5 & 9.8 & 3.7 & 75.4 & 9.2 & 15.4 \\
\hline 3 Gy PBS & 66.2 & 17.6 & 16.2 & 68.0 & 15.7 & 16.3 & 60.9 & 19.6 & 19.5 \\
\hline 3 Gy Ad-IU2 & 75.9 & 18.3 & 5.8 & 78.3 & 15.4 & 6.3 & 70.7 & 11.3 & 18.0 \\
\hline 3 Gy Ad-E4 & 64.9 & 19.1 & 16.0 & 71.3 & 13.3 & 15.4 & 66.9 & 9.8 & 23.3 \\
\hline 3 Gy rhTRAIL & 70.2 & 21.3 & 8.5 & 76.2 & 15.8 & 8.0 & 73.5 & 12.6 & 13.9 \\
\hline
\end{tabular}



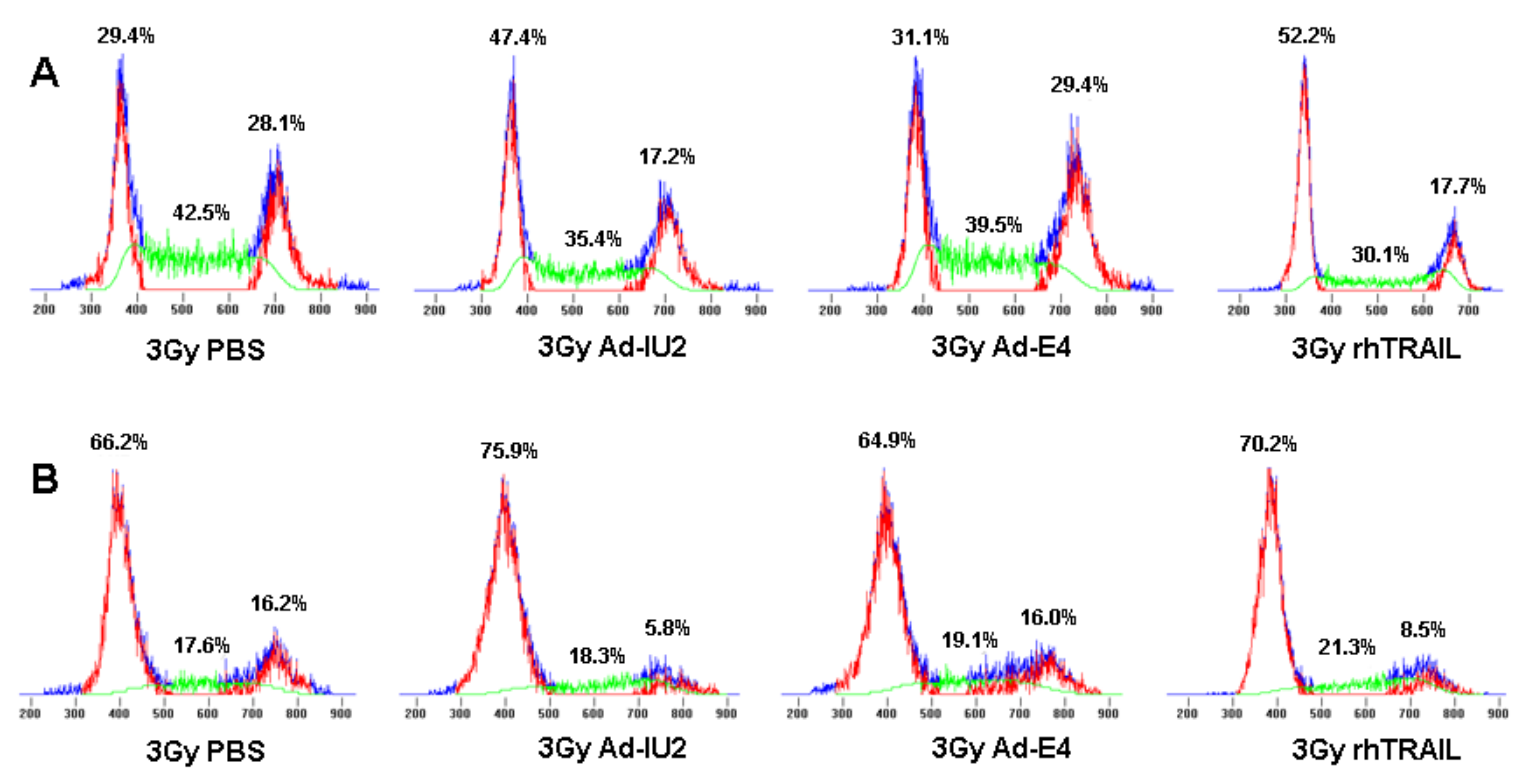

Figure 27. Combination of Ad-IU2 or rhTRAIL and EBRT resulted in perturbation of RTinduced $G_{2}$ phase arrest. CWR22rv (A) and C4-2 (B) cells were treated with PBS, AdIU2, Ad-E4PSESE1a or rhTRAIL for 24 hours, followed by treatment with 3 Gy RT. 8 hours after irradiation, cells were harvested and stained for DNA content with PI. Percent of cells in each phase of the cell cycle is listed for each histogram. The radiation-induced $\mathrm{G}_{2}$ arrest was dramatically diminished in cells pretreated with Ad-IU2 or rhTRAIL. 


\section{Discussion}

The safety and efficacy of gene therapy for prostate cancer has been demonstrated through various preclinical and clinical trials. In recent years, interest in this field has expanded and will continue to do so. Preliminary results from clinical trials indicate that gene therapy alone may not cure prostate cancer; however, in combination with conventional therapies, gene therapy promises to fill the therapeutic void left by chemotherapy and androgen ablation therapy for advanced disease. It is conceivable that such multi-modal therapies will result in fewer undesirable side effects and improvement in the quality of life for the patient. Due to its convenient administration through ultrasound-guided transrectal injection, it is also conceivable that gene therapy, applied in an outpatient setting, may one day augment radical prostatectomy and radiation therapy to treat early stage disease. Factors impeding the widespread use of gene therapy for prostate cancer include slow clinical translation of laboratory research, lack of funding for expensive clinical trials, and misconceptions and fear of gene therapy by the general public. As the number of successful gene therapy trials increase, these factors will diminish.

Significant progress in basic medical science research has lead to an understanding of the molecular events underlying the development and progression of prostate cancer. Such preclinical studies have translated into strategies for the molecular therapy of hormone-refractory prostate cancer. Advances in molecular and cellular biology have lead to the discovery of novel therapeutic genes and improvement of vector systems for the delivery of those genes. Prostate-specific targeting of molecular therapies is now possible due to the identification and characterization of prostate-specific sequences. Our laboratory has focused much of its efforts on the development of chimeric tissue-specific promoters such as PSES and the application of 
well-characterized promoters such as $\mathrm{OC}$ in the development of molecular therapy for androgen-independent primary and metastatic prostate cancer.

Early gene therapy clinical trials for cancer involved replication-deficient adenoviral vectors due to safety concerns of nonspecific viral replication in immunocompromised patients; however, these studies were limited by poor viral transduction efficiencies. To overcome this, replication-competent oncolytic adenoviral vectors were developed to kill cancer cells directly and further propagate the vector. The first tumor-specific oncolytic adenovirus developed was ONYX-015, in which E1b-55kd was deleted to restrict replication to p53-deficient cancer cells (177); however, later studies have demonstrated replication independent of p53 status (189). ONYX-015 has been widely tested in clinical trials and has demonstrated inefficient cell lysis and viral replication, resulting in poor clinical outcomes $(180,301)$. In a second approach, the adenoviral immediate early E1a gene was placed under control of tissue-specific promoters $(193,198,302)$. To achieve greater control of viral replication, both E1a and E1b genes were placed under control of multiple or single bidirectional promoters (195, 199). We developed a prostate-specific oncolytic adenovirus, called Ad-E4PSESE1a, in which adenoviral E1a and E4 genes were controlled by the bidirectional PSES enhancer (205). Previous studies have demonstrated that TRAIL-mediated apoptosis potentiated oncolysis and spread of a replication-competent adenovirus throughout a tumor (303). Therefore, to enhance the oncolytic and therapeutic potential of this PSRCA, I incorporated TRAIL cDNA under the transcriptional control of PSES, making Ad-IU2 the first prostate-specific TRAIL vector.

Currently, four strategies have been developed to deliver TRAIL via conditionallyreplicating adenoviral vectors. Ad5/35.IR-E1a/TRAIL is an adenovirus containing an inverted E1a sequence that replicated exclusively in tumor cells via complementation with unknown cellular factors (304). Through an adenoviral replication-dependent 
homologous recombination event, the bicistronic 3' to 5' TRAIL-IRES-E1a cassette is flipped to the correct orientation and expressed under control of the universal RSV promoter (83). In a second tumor-specific approach, a CMV-TRAIL expression cassette was incorporated into the E1b-55kd-deleted adenovirus, ONYX015. To improve the anti-tumor efficacy of this virus, ZD55-hTRAIL, against colorectal carcinoma, it was administered in combination with 5-FU (305). Ad/TRAIL-E1 contains two duplicated synthetic promoters in close proximity comprised of the full hTERT promoter and minimal sequences from the CMV promoter to control the expression of both E1a and TRAIL as separate transcripts (306). The final approach achieved tissue-specific replication and TRAIL expression for hepatocellular carcinoma using the $\alpha$-fetoprotein promoter to control the expression of a bicistronic E1a-TRAIL cassette (307). My strategy is unique to the previously described replication-competent TRAIL vectors in that tight tissue-specific regulation of adenoviral replication was achieved by controlling three early adenoviral genes, E1a, E1b and E4. Furthermore, in my study TRAIL was co-expressed with adenoviral E1a in PSA/PSMA-positive cells without the use of bicistronic elements, which can result in decreased gene expression of transgenes downstream of the internal ribosomal entry sequence (IRES), or universal promoters, which may induce adenoviral replication outside of the target tissue. The use of a prostate-specific promoter such as PSES to control viral replication and transgene expression enhanced the safety of this vector, while enabling its use for both locally advanced and metastatic prostate cancer.

We hypothesized that arming a prostate-specific oncolytic adenoviral vector with TRAIL would enhance the anti-tumor efficacy of a replication-competent adenovirus. In this approach, prostate cancer cells would undergo apoptosis by the tumor-specific apoptosis inducer, TRAIL. Furthermore, the replication of the adenoviral vector would kill prostate cancer cells directly by oncolysis and result in amplification of both 
adenoviral vector and viral transduction efficiency. Finally, through a TRAIL-mediated bystander effect, cytotoxicity of this molecular therapy would spread throughout the tumor, killing nontransduced cells.

In this study, I developed and characterized Ad-IU2, a novel PSRCA, in which adenoviral replication and the expression of TRAIL were controlled by the prostatespecific PSES promoter. I demonstrated that the adenoviral early gene product, E1a, which controls adenoviral replication, was restricted to PSA/PSMA-positive prostate cancer cells (Figure 8). Likewise, replication of Ad-IU2 was diminished in PSA/PSMAnegative cells (Table 4). While TRAIL was expressed in PSA/PSMA-positive prostate cancer cells (Figures 6 and 7), this did not hinder viral replication. This was consistent with my ability to produce high titers of Ad-IU2 $\left(1.02 \times 10^{12} \mathrm{vp} / \mathrm{ml}\right)$. Other groups have had difficulty producing adenoviral vectors expressing TRAIL or similar death ligands due to toxicity to producer cells such as HEK293 or HER911. Previous attempts have used caspase inhibitors to allow adenoviral replication without the premature death of producer cells (277). In my approach, the unintended death of HER911E4 producer cells is prevented by tissue-specific regulation of TRAIL expression.

Ad-IU2 effectively induced apoptosis in TRAIL-sensitive, PSA/PSMA-positive CWR22rv and C4-2 cells (Figure 9). Interestingly, TRAIL resistance in the PSA/PSMApositive prostate cancer cell line LNCaP was reversed by prolonged Ad-IU2 infection (Figure 10). Ad-IU2 exhibited marked in vitro killing activity against both androgendependent and androgen-independent PSA/PSMA-positive prostate cancer cells, compared to the PSRCA, Ad-E4PSESE1a, suggesting that the addition of TRAIL to a PSRCA augmented the killing power of a replication-competent adenovirus (Figure 11AC). The ability to maintain strong PSES activity regardless of androgen status will allow Ad-IU2 to be used for either early or advanced prostate tumors. TRAIL is a valuable anti-tumor molecular therapeutic agent because of its preference for inducing apoptosis 
in cancer cells. Retaining this ability, Ad-IU2 failed to kill PSA/PSMA-negative normal human dermal fibroblasts (Figure 11D).

Due to the heterogeneity of cells within a single tumor of the prostate, with respect to PSA, PSMA, AR and CAR expression, as well as the reduced in vivo transduction efficiency of adenoviral vectors, the ability of Ad-IU2 gene therapy to induce a bystander killing effect is critical to its success as a therapeutic agent. In co-culture with Ad-IU2-infected PSA/PSMA-positive CWR22rv cells, apoptosis was detected in PSA/PSMA-negative PC-3 cells, implicating a potential role for cell-to-cell contact with Ad-IU2-infected cells expressing surface-bound TRAIL (Figure 12). Similarly, apoptosis was detected in both CWR22rv and PC-3 cells treated with conditioned medium from Ad-IU2-infected cells which had been heat-inactivated to deplete viable Ad-IU2 virus particles from the medium (Figure 13). However, western blot analysis of the conditioned medium revealed that soluble TRAIL was not cleaved from the cell membranes of Ad-IU2-infected cells (Figure 15), suggesting that other soluble factors, such as TRAIL-containing apoptotic bodies or even non-TRAIL proteins, were responsible for the bystander killing effect.

Ad-IU2 significantly suppressed the in vivo growth of androgen-independent CWR22rv xenografts in nude athymic mice compared to a PSRCA control (3.1 vs. 17.1fold growth of tumor). At the 6-week endpoint of the study, 6 tumors responded favorably with partial regression in 4 of 6 or complete regression in 2 of 6 tumors. Of the 3 tumors that failed treatment, two were still significantly suppressed compared to PSRCA-treated tumors (Figures 16 and 17). The histological appearance of Ad-IU2treated tumors was drastically different than PSRCA-treated tumors. In the Ad-IU2 treatment group, the treatment effect appeared to extend beyond the necrotic viral centers of replication, whereas in the PSRCA control group, the treatment effect was limited to the margin of cells surrounding viral centers of replication (Figure 18), 
demonstrating enhanced therapeutic effect and viral spread throughout a greater tumor volume. The use of the highly tissue-specific PSES promoter will not only for allow intratumoral injection of the vector for locally advanced or recurrent tumors, but also systemic administration of the virus to target distant metastases; however, the greatest limitation of this approach to be overcome is the dilution effect of the virus in the bloodstream. Of the prostate-specific promoters available, PSES retains the highest tissue-specificity and activity in environments depleted of androgens, giving this PSRCA greater clinical utility in patients undergoing simultaneous ADT. Although gene therapy with Ad-IU2 shows favorable preclinical promise as a therapeutic agent for advanced androgen-independent prostate cancer, its use as a monotherapy may not be feasible. Ad-IU2 viral therapy would benefit greatly from combination with conventional therapies such as radiation therapy.

Recently, it has been demonstrated that an enhanced anti-tumor effect can be achieved when rhTRAIL therapy is combined with EBRT. I hypothesized that the neoadjuvant treatment of high risk prostate cancer with a PSRCA expressing TRAIL would result in the radio-sensitization of tumor cells, thereby enhancing the therapeutic effect of EBRT. This approach is ideal over the use of rhTRAIL to sensitize tumors to radiotherapy because replication-competent vectors can achieve enhanced distribution throughout the entire tumor. Furthermore, through the use of a tissue-specific promoter such as PSES, radio-sensitization can be limited to prostate cancer cells, minimizing toxicity to normal tissues.

EBRT is viewed as the standard of care for high risk locally advanced prostate cancer; however, clinical outcomes are merely intermediate to moderate, despite combination with ADT. In order to achieve a long-term cure, the local disease must be controlled; however, the persistence of radio-resistant cancer cells and RT dose-limiting toxicities prevent the complete elimination of all tumor cells. For this reason, a treatment 
modality that enhances the therapeutic efficacy of EBRT within the local tumor environment and effectively reduces the required dose of radiation administered is quite appealing.

In this study, the pretreatment of androgen-independent CWR22rv and C4-2 prostate cancer cells with $0.003 \mathrm{LDU} /$ cell resulted in significant radio-sensitization as evidenced by a reduction in the shoulder of the radiation survival curves, an increase in lethal DNA damage ( $\alpha)$, and a decrease in sublethal events $(\beta)$. Untreated C4-2 cells were found to be more radio-sensitive than CWR22rv cells, with a mean lethal dose of 2.35 Gy, compared to 2.52 Gy. Ad-IU2 had the greatest radio-sensitizing effect on CWR22rv cells $(\mathrm{DRF}=4.91)$, compared to C4-2 $(\mathrm{DRF}=2.43)$. In both cell lines, Ad-IU2 was a significantly better radiation-sensitizing agent than $75 \mathrm{ng} / \mathrm{ml}$ rhTRAIL (Figures 21 and 22). Radio-sensitization by Ad-IU2 did not occur in PSA/PSMA-negative LoVo and T24 cells (Figure 24). Because these cell lines differ genetically from normal cells, they may not behave similarly. Therefore, a limitation of this study is that the radiosensitization of primary urothelial, colorectal and bladder smooth muscle cells was not tested; however, the difficulty of performing clonogenic assays on primary cells precludes this method. These data suggest that Ad-IU2 is a potent radio-sensitizer in PSA/PSMA-positive androgen-independent prostate cancer cells that will result in enhancement of the RT effect and radiation dose-reduction. Through the tight control of TRAIL expression by the PSES promoter, toxicity to surrounding normal tissues should also be reduced. Although the in vitro Ad-IU2 data show promise as a radio-sensitizing agent for clinical use, no radio-sensitizing data in similar cell lines exist for comparison. Radiation-sensitizers for prostate cancer have not been widely evaluated in the laboratory. Currently, the use of docetaxel in combination with EBRT is being investigated clinically. The combinatorial therapy was tolerated well, and the maximum tolerable dose has not yet been met (290). 
Several mechanisms could explain or contribute to the enhancement of the therapeutic effect when molecular therapy with TRAIL is combined with EBRT. First, ionizing radiation may upregulate the expression of DR4 or DR5, the cell surface receptors for TRAIL. Such an increase in TRAIL receptor may enhance TRAIL tumor killing by reversing resistance to TRAIL. Secondly, concurrent treatment with RT may enhance expression of TRAIL throughout the tumor, as it has been shown that ionizing radiation improves the transduction efficiency and transgene integration of adenoviral vectors $(308,309)$. In addition, TRAIL may kill residual radio-resistant prostate cancer cells that remain after treatment with EBRT. Next, it is known that TRAIL and RT act upon distinct pathways to trigger apoptosis, TRAIL through an extrinsic caspasemediated pathway and ionizing radiation through an intrinsic mitochondrial-dependent pathway. Therefore, the combination of TRAIL receptor stimulation and radiation should activate both pathways, resulting in an amplification of apoptosis. Previous studies have demonstrated this effect in cells treated with TRAIL molecular therapy and EBRT; however, this effect was only seen at very high doses of EBRT $(294,295,299)$. In the present study, at the minimal doses of Ad-IU2 and EBRT required to reduce the clonogenic survival of CWR22rv and C4-2 cells, enhancement of apoptosis induction with combinatorial therapy was not observed (Figure 25). This suggests that a different mechanism exists for radio-sensitization in prostate cancer cells treated with Ad-IU2.

Cell cycle analysis of prostate cancer cells infected with Ad-IU2 or pretreated with rhTRAIL for 24 hours revealed a $20 \%$ increase in the $\mathrm{G}_{1}$ population for both CWR22rv and C4-2 cells (Figure 26). This may contribute to the mechanism of radiosensitization, as cells arrested in $G_{1}$ or $G_{2}$ phases are most sensitive to RT. Following combination with EBRT, this effect was still observed for 8 hours after irradiation in CWR22rv cells or 24 hours in C4-2 cells. This cell cycle redistribution also resulted in a reduction of the radio-resistant $S$ phase. The most striking cell cycle effect was 
observed following combinatorial therapy where pretreatment of prostate cancer cells with Ad-IU2 or rhTRAIL resulted in an decrease in the RT-induced $\mathrm{G}_{2}$ arrest. At 8 hours after irradiation, a $40 \%$ reduction in the $\mathrm{G}_{2}$ arrest was observed in CWR22rv cells and a $55 \%$ reduction was observed in C4-2 cells (Figure 27). This effect lasted up to 8 hours for CWR22rv cells and up to 16 hours for C4-2 cells (Table 8). The mitotic checkpoint is in place to prevent cells with significant chromosomal damage from exiting mitosis. Perturbation of the RT-induced $\mathrm{G}_{2}$ arrest is significant because it can lead to genomic instability and mitotic catastrophe, playing a significant role in the radio-sensitization of cells and contributing to the death of irradiated prostate cancer cells. 


\section{Future Directions}

Within the last several years, studies in the field of cancer gene therapy have attempted to improve gene delivery vectors by creating viruses with the ability to package a greater number of transgenes, hide from the host's immune system, deliver transgenes with greater transduction efficiency, and target tumor cells selectively. Since the cloning and initial production of Ad-IU2, many of these improvements have been well characterized. As with most agents currently under investigation in clinical trials, an improved version of the therapeutic modality is usually available in the laboratory, but less thoroughly studied. By manipulation of the adenoviral genome, such improvements can be made to Ad-IU2 as well. These vector improvements might enhance the therapeutic efficacy of Ad-IU2 as a single agent or in combination with EBRT.

Perhaps the most beneficial vector modification would be the deletion of the E3 adenoviral region. This early adenoviral gene encodes several gene products that prevent or diminish apoptosis induction in the host cell and are not critical for viral replication (67). If the E3 region is deleted, the adenoviral late coding sequence ADP, which is found within the E3 region, should be reinserted to enhance cell lysis and release of viral progeny (72). In the current study, although Ad-IU2 induced apoptosis significantly above that of a PSRCA, levels of apoptosis achieved were no greater than $25 \%$ at 24 hours after infection (Figure 9). These low levels of apoptosis induction may be the result of adenoviral anti-apoptotic proteins. The resulting deletion of the E3 region and reinsertion of the ADP coding sequence would also provide an additional 2 $\mathrm{kb}$ of packaging space. A second modification would be to substitute the Ad5 fiber knob from Ad-IU2 with the fiber knob from Ad35. This improvement would result in greater infectivity in prostate cancer cells, decreased viral tropism for hepatic cells, and reduction in innate immunity against the vector, because neutralizing antibodies against 
Ad35 are less common in patients than against Ad5 (81). In addition, this modification would result in a gain of $756 \mathrm{bp}$ of genome space for more therapeutic inserts. With the approximately $2.8 \mathrm{~kb}$ of gained insert space, an additional transgene could be inserted such as the anti-angiogenic factor, EndoAngio (211). One final consideration for a vector modification would be to replace one PSES sequence for another prostatespecific promoter such as OC. Because Ad-IU2 contains two copies of PSES, one near each ITR, although rare, there exists the risk that a homologous recombination event might occur between the sequences in an infected cell, resulting in excision from the genome and loss of the therapeutic vector.

One further question remains to be answered regarding the evaluation of the Ad-IU2 bystander effect. Is the bystander effect that is observed in conditioned medium from Ad-IU2-infected cells due to apoptotic bodies containing TRAIL on the membrane surface or other soluble factors released from infected cells? To begin to answer this, the conditioned medium from Ad-IU2-infected cells could be applied to LNCaP cells, which are highly resistant to TRAIL. The induction of apoptosis in LNCaP cells could indicate that soluble factors, other than apoptotic bodies, are responsible for the bystander effect. However, perhaps a better experimental method would be to use antiTRAIL receptor blocking antibodies in TRAIL-sensitive cells treated with conditioned medium. A reduction or complete blockade of apoptosis would suggest that TRAILcontaining apoptotic bodies played a role in or were entirely responsible for the bystander effect. If apoptosis induction was not affected by TRAIL-blocking antibodies, this would suggest a role for other soluble factors released from Ad-IU2-infected cells.

Another area of investigation is the study of the molecular events responsible for the cell cycle changes that occurred in prostate cancer cells treated with combinatorial therapy. Treatment of prostate cancer cells with Ad-IU2 or rhTRAIL for 24 hours resulted in arrest of cells in $\mathrm{G}_{1}$ phase, a stage in the cell cycle in which cells are more 
radio-sensitive. Following combinatorial therapy, cells pretreated with Ad-IU2 or rhTRAIL demonstrated a diminished RT-induced $\mathrm{G}_{2}$ arrest. It was recently discovered that FADD was required for the combined effect of TRAIL and RT (310). Nuclear localization (311) and phosphorylation of FADD at serine 194 by casein kinase la (CKla) (312) has also been shown to be essential for cell cycle regulation. It is known that FADD is dephosphorylated in $G_{1}$ phase; however, its role in regulating progression through the $G_{1} / S$ checkpoint is unclear (313). Further investigation is warranted to determine whether FADD is responsible for the accumulation of cells in $G_{1}$ phase following Ad-IU2 or rhTRAIL treatment. In prostate cancer cells, it was discovered that Ser 194-phosphorylated nuclear FADD contributed to $G_{2}$ arrest of cells (314). It is possible that activation of the DISC and sequestration of FADD to the cell membrane would result in a decrease in the amount of FADD available in the nucleus, leading to an abrogation of the RT-induced $\mathrm{G}_{2}$ arrest. To better understand the role of FADD in cell cycle regulation, the nuclear sublocalization and phosphorylation of FADD should be studied in prostate cancer cells following combinatorial therapy. Finally, a second possible mechanism for the perturbation of $G_{2}$ arrest may be caspase-dependent degradation of mitotic checkpoint proteins. Kim et al. recently described a similar $G_{2}$ arrest abrogation when rhTRAIL was combined with the microtubule-targeting drugs nocodazole or paclitaxel. The combination of drugs resulted in the degradation of the mitotic checkpoint proteins BubR1 and Bub1 by activated caspases (315). A screen of several $G_{1} / S$ and $G_{2} / M$ checkpoint proteins following the combinatorial treatment of prostate cancer cells with Ad-IU2 and EBRT may be useful to determine whether caspase-mediated cleavage of check point proteins was responsible for the radiosensitization of prostate cancer cells by Ad-IU2 and rhTRAlL. However, one should also consider that arrest of cells in $G_{1}$ alone may be responsible for the decrease in the $G_{2}$ population, due to fewer cycling cells. Synchronization experiments may be necessary 
to further understand the role of cell cycle in the radio-sensitization of prostate cancer cells. A working hypothesis model for the sensitization of prostate cancer cells to radiation therapy by Ad-IU2 is represented in Figure 28.

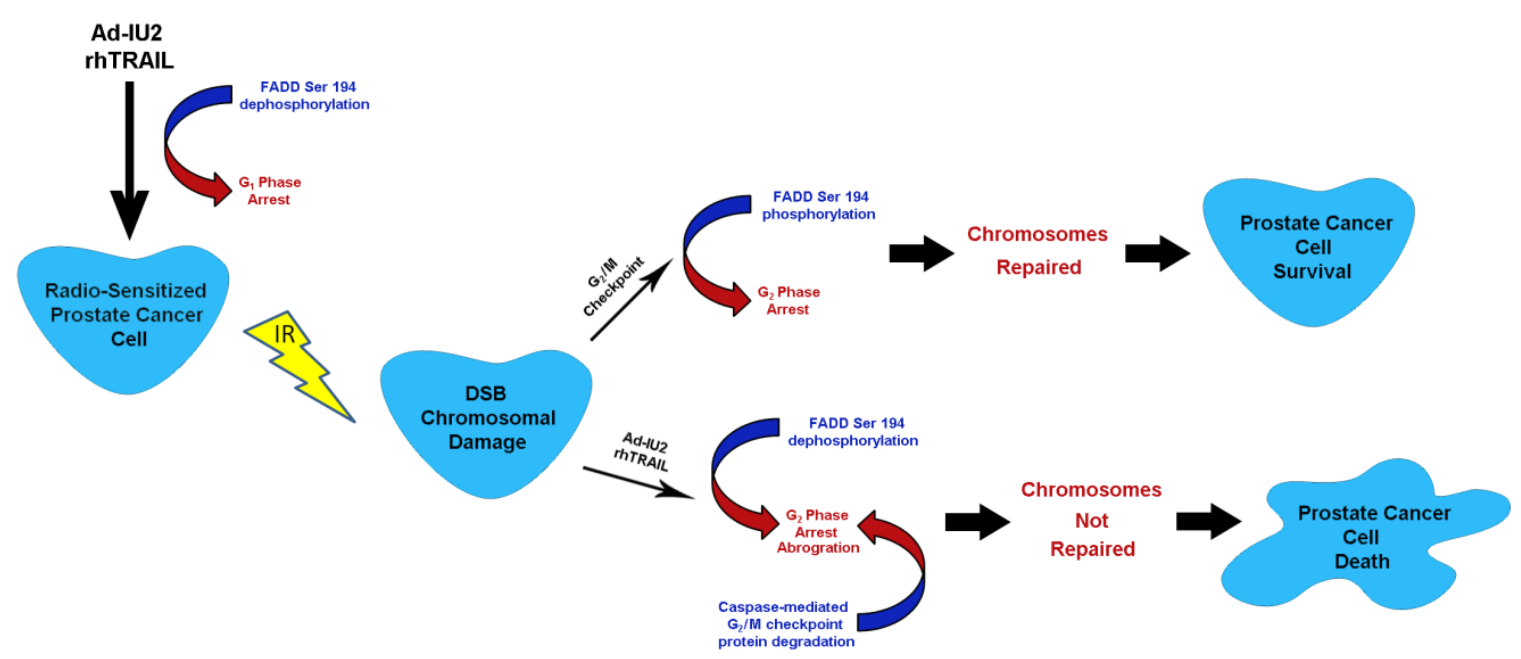

Figure 28. Hypothetical mechanism of radio-sensitization by Ad-IU2. Exposure of prostate cancer cells to TRAIL by Ad-IU2 infection or rhTRAIL administration resulted in $\mathrm{G}_{1}$ phase arrest. One possible mechanism for this is the sequestration of FADD to the cell membrane, preventing its phosphorylation at serine residue 194. In an untreated prostate cancer cell, exposure to ionizing radiation activates the $G_{2} / M$ checkpoint, induces FADD phosphorylation and causes $\mathrm{G}_{2}$ arrest, allowing sufficient time to repair double strand breaks (DSB) in chromosomes. In prostate cancer cells pretreated with Ad-IU2 or rhTRAIL, the RT-induced $G_{2}$ arrest was diminished. Two possible mechanisms may explain this cell cycle alteration. First, activation of the caspase cascade by TRAIL may induce degradation of $\mathrm{G}_{2} / \mathrm{M}$ checkpoint proteins, allowing cells to progress without chromosomal repair. The second method involves the reduction of phosphorylated FADD due to sequestration of the DISC at the cell membrane, resulting in progression through the $\mathrm{G}_{2} / \mathrm{M}$ checkpoint and a mitotic catastrophe. 
The final area of investigation remaining in this study is to test the in vivo efficacy of combinatorial therapy against androgen-independent CWR22rv tumor xenografts in nude mice. This is necessary to confirm the in vitro radio-sensitizing properties of AdIU2 and will require dosimetry experiments on SQ CWR22rv tumors to determine the proper in vivo RT dose. Once this has been achieved, a combinatorial treatment regimen must be designed and evaluated to achieve EBRT dose reduction. This animal experiment will serve as the proof-of-principle for clinical evaluation of the therapy. To assess the safety of this multi-modal therapy, a toxicology study should be performed in mice evaluating the safety of the vector alone and in combination with EBRT, looking for toxicity to major organ systems including cardiovascular, hematologic, central nervous, pulmonary, renal and hepatobiliary. The results obtained from the in vitro study showed great promise for translation to a clinical trial. After successful completion of the toxicology study, IND status should be obtained and a clinical protocol developed and filed with the RAC for the combinatorial treatment of locally advanced disease or spinal metastatic lesions through intratumoral injection of Ad-IU2 and co-administration of EBRT. 


\section{References}

1. Jemal A, Siegel R, Ward E, et al. Cancer statistics, 2008. CA Cancer J Clin 2008 Mar-Apr;58(2):71-96.

2. Isaacs JT. Molecular markers for prostate cancer metastasis. Developing diagnostic methods for predicting the aggressiveness of prostate cancer. Am J Pathol 1997 May;150(5):1511-21.

3. Dijkman GA, Debruyne FM. Epidemiology of prostate cancer. Eur Urol 1996;30(3):281-95.

4. Meikle AW, Smith JA, Jr. Epidemiology of prostate cancer. Urol Clin North Am 1990 Nov;17(4):709-18.

5. Amling $\mathrm{CL}$, Riffenburgh $\mathrm{RH}$, Sun $\mathrm{L}$, et al. Pathologic variables and recurrence rates as related to obesity and race in men with prostate cancer undergoing radical prostatectomy. J Clin Oncol 2004 Feb 1;22(3):439-45.

6. Freedland SJ, Aronson WJ, Kane CJ, et al. Impact of obesity on biochemical control after radical prostatectomy for clinically localized prostate cancer: a report by the Shared Equal Access Regional Cancer Hospital database study group. J Clin Oncol 2004 Feb 1;22(3):446-53.

7. Platz EA, Leitzmann MF, Michaud DS, Willett WC, Giovannucci E. Interrelation of energy intake, body size, and physical activity with prostate cancer in a large prospective cohort study. Cancer Res 2003 Dec 1;63(23):8542-8.

8. Bosetti C, Micelotta S, Dal Maso L, et al. Food groups and risk of prostate cancer in Italy. Int J Cancer 2004 Jun 20;110(3):424-8.

9. Colli JL, Colli A. International comparisons of prostate cancer mortality rates with dietary practices and sunlight levels. Urol Oncol 2006 May-Jun;24(3):184-94.

10. Talamini R, Franceschi S, La Vecchia C, Serraino D, Barra S, Negri E. Diet and prostatic cancer: a case-control study in northern Italy. Nutr Cancer 1992;18(3):277-86.

11. West DW, Slattery ML, Robison LM, French TK, Mahoney AW. Adult dietary intake and prostate cancer risk in Utah: a case-control study with special emphasis on aggressive tumors. Cancer Causes Control 1991 Mar;2(2):85-94.

12. Lichtenstein P, Holm NV, Verkasalo PK, et al. Environmental and heritable factors in the causation of cancer--analyses of cohorts of twins from Sweden, Denmark, and Finland. N Engl J Med 2000 Jul 13;343(2):78-85.

13. Smith JR, Freije D, Carpten JD, et al. Major susceptibility locus for prostate cancer on chromosome 1 suggested by a genome-wide search. Science 1996 Nov $22 ; 274(5291): 1371-4$.

14. Chamberlain NL, Driver ED, Miesfeld RL. The length and location of CAG trinucleotide repeats in the androgen receptor $\mathrm{N}$-terminal domain affect transactivation function. Nucleic Acids Res 1994 Aug 11;22(15):3181-6.

15. Kazemi-Esfarjani P, Trifiro MA, Pinsky L. Evidence for a repressive function of the long polyglutamine tract in the human androgen receptor: possible pathogenetic relevance for the (CAG)n-expanded neuronopathies. Hum Mol Genet 1995 Apr;4(4):5237.

16. Nam RK, Toi A, Vesprini D, et al. V89L polymorphism of type-2, 5-alpha reductase enzyme gene predicts prostate cancer presence and progression. Urology 2001 Jan;57(1):199-204. 
17. Herman CM, Kattan MW, Ohori M, Scardino PT, Wheeler TM. Primary Gleason pattern as a predictor of disease progression in gleason score 7 prostate cancer: a multivariate analysis of 823 men treated with radical prostatectomy. Am J Surg Pathol 2001 May;25(5):657-60.

18. D'Amico AV, Chen MH, Roehl KA, Catalona WJ. Preoperative PSA velocity and the risk of death from prostate cancer after radical prostatectomy. N Engl J Med 2004 Jul 8;351(2):125-35.

19. D'Amico AV, Moul JW, Carroll PR, Sun L, Lubeck D, Chen MH. Surrogate end point for prostate cancer-specific mortality after radical prostatectomy or radiation therapy. J Natl Cancer Inst 2003 Sep 17;95(18):1376-83.

20. D'Amico AV, Whittington R, Malkowicz SB, et al. Biochemical outcome after radical prostatectomy, external beam radiation therapy, or interstitial radiation therapy for clinically localized prostate cancer. JAMA 1998 Sep 16;280(11):969-74.

21. Thompson KE, Hernandez J, Canby-Hagino ED, Troyer D, Thompson IM. Prognostic features in men who died of prostate cancer. J Urol 2005 Aug;174(2):553-6; discussion 6.

22. Nelson JE, Harris RE. Inverse association of prostate cancer and non-steroidal anti-inflammatory drugs (NSAIDs): results of a case-control study. Oncol Rep 2000 JanFeb;7(1):169-70.

23. Nelson WG, De Marzo AM, Deweese TL, et al. Preneoplastic prostate lesions: an opportunity for prostate cancer prevention. Ann N Y Acad Sci 2001 Dec;952:135-44.

24. Lin X, Tascilar M, Lee WH, et al. GSTP1 CpG island hypermethylation is responsible for the absence of GSTP1 expression in human prostate cancer cells. Am J Pathol 2001 Nov;159(5):1815-26.

25. Brooks JD, Weinstein M, Lin X, et al. CG island methylation changes near the GSTP1 gene in prostatic intraepithelial neoplasia. Cancer Epidemiol Biomarkers Prev 1998 Jun;7(6):531-6.

26. Bieberich CJ, Fujita K, He WW, Jay G. Prostate-specific and androgendependent expression of a novel homeobox gene. J Biol Chem 1996 Dec 13;271(50):31779-82.

27. Chen H, Nandi AK, Li X, Bieberich CJ. NKX-3.1 interacts with prostate-derived Ets factor and regulates the activity of the PSA promoter. Cancer Res 2002 Jan 15;62(2):338-40.

28. Bowen C, Bubendorf L, Voeller HJ, et al. Loss of NKX3.1 expression in human prostate cancers correlates with tumor progression. Cancer Res 2000 Nov 1;60(21):6111-5.

29. Tomlins SA, Rhodes DR, Perner S, et al. Recurrent fusion of TMPRSS2 and ETS transcription factor genes in prostate cancer. Science 2005 Oct 28;310(5748):644-8.

30. Perner S, Mosquera JM, Demichelis F, et al. TMPRSS2-ERG fusion prostate cancer: an early molecular event associated with invasion. Am J Surg Pathol 2007 Jun;31(6):882-8.

31. McMenamin ME, Soung P, Perera S, Kaplan I, Loda M, Sellers WR. Loss of PTEN expression in paraffin-embedded primary prostate cancer correlates with high Gleason score and advanced stage. Cancer Res 1999 Sep 1;59(17):4291-6.

32. Suzuki H, Freije D, Nusskern DR, et al. Interfocal heterogeneity of PTEN/MMAC1 gene alterations in multiple metastatic prostate cancer tissues. Cancer Res 1998 Jan 15;58(2):204-9.

33. Koivisto P, Kononen J, Palmberg C, et al. Androgen receptor gene amplification: a possible molecular mechanism for androgen deprivation therapy failure in prostate cancer. Cancer Res 1997 Jan 15;57(2):314-9. 
34. Craft N, Shostak Y, Carey M, Sawyers CL. A mechanism for hormoneindependent prostate cancer through modulation of androgen receptor signaling by the HER-2/neu tyrosine kinase. Nat Med 1999 Mar;5(3):280-5.

35. Culig Z, Hobisch A, Cronauer MV, et al. Mutant androgen receptor detected in an advanced-stage prostatic carcinoma is activated by adrenal androgens and progesterone. Mol Endocrinol 1993 Dec;7(12):1541-50.

36. Shi XB, Ma AH, Xia L, Kung HJ, de Vere White RW. Functional analysis of 44 mutant androgen receptors from human prostate cancer. Cancer Res 2002 Mar 1;62(5):1496-502.

37. Carver BS, Bianco FJ, Jr., Scardino PT, Eastham JA. Long-term outcome following radical prostatectomy in men with clinical stage T3 prostate cancer. J Urol 2006 Aug;176(2):564-8.

38. Ward JF, Slezak JM, Blute ML, Bergstralh EJ, Zincke H. Radical prostatectomy for clinically advanced (cT3) prostate cancer since the advent of prostate-specific antigen testing: 15-year outcome. BJU Int 2005 Apr;95(6):751-6.

39. Bolla $M$, van Poppel $H$, Collette $L$, et al. Postoperative radiotherapy after radical prostatectomy: a randomised controlled trial (EORTC trial 22911). Lancet 2005 Aug 1319;366(9485):572-8.

40. Huggins C, Hodges CV. Studies on prostatic cancer. I. The effect of castration, of estrogen and androgen injection on serum phosphatases in metastatic carcinoma of the prostate. CA Cancer J Clin 1972 Jul-Aug;22(4):232-40.

41. Crawford ED, Eisenberger MA, McLeod DG, et al. A controlled trial of leuprolide with and without flutamide in prostatic carcinoma. N Engl J Med 1989 Aug 17;321(7):419-24.

42. Eisenberger MA, Blumenstein BA, Crawford ED, et al. Bilateral orchiectomy with or without flutamide for metastatic prostate cancer. N Engl J Med 1998 Oct 8;339(15):1036-42.

43. Coleman CN. International Conference on Translational Research and Preclinical Strategies in Radio-Oncology (ICTR)--conference summary. Int J Radiat Oncol Biol Phys 2001 Feb 1;49(2):301-9.

44. Osborn JL, Getzenberg RH, Trump DL. Spinal cord compression in prostate cancer. J Neurooncol 1995;23(2):135-47.

45. Hanks GE, Hanlon AL, Epstein B, Horwitz EM. Dose response in prostate cancer with 8-12 years' follow-up. Int J Radiat Oncol Biol Phys 2002 Oct 1;54(2):427-35.

46. Hanks GE, Hanlon AL, Pinover WH, Horwitz EM, Schultheiss TE. Survival advantage for prostate cancer patients treated with high-dose three-dimensional conformal radiotherapy. Cancer J Sci Am 1999 May-Jun;5(3):152-8.

47. O'Connor KM, Fitzpatrick JM. Side-effects of treatments for locally advanced prostate cancer. BJU Int 2006 Jan;97(1):22-8.

48. Roach M, 3rd. Reducing the toxicity associated with the use of radiotherapy in men with localized prostate cancer. Urol Clin North Am 2004 May;31(2):353-66.

49. Fuks Z, Leibel SA, Wallner KE, et al. The effect of local control on metastatic dissemination in carcinoma of the prostate: long-term results in patients treated with 125I implantation. Int J Radiat Oncol Biol Phys 1991 Aug;21(3):537-47.

50. Coen JJ, Zietman AL, Thakral H, Shipley WU. Radical radiation for localized prostate cancer: local persistence of disease results in a late wave of metastases. J Clin Oncol 2002 Aug 1;20(15):3199-205.

51. Worgall S, Wolff G, Falck-Pedersen E, Crystal RG. Innate immune mechanisms dominate elimination of adenoviral vectors following in vivo administration. Hum Gene Ther 1997 Jan 1;8(1):37-44. 
52. Chirmule N, Propert K, Magosin S, Qian Y, Qian R, Wilson J. Immune responses to adenovirus and adeno-associated virus in humans. Gene Ther 1999 Sep;6(9):157483.

53. Schiedner G, Morral N, Parks RJ, et al. Genomic DNA transfer with a highcapacity adenovirus vector results in improved in vivo gene expression and decreased toxicity. Nat Genet 1998 Feb;18(2):180-3.

54. Hearing $P$, Samulski RJ, Wishart WL, Shenk T. Identification of a repeated sequence element required for efficient encapsidation of the adenovirus type 5 chromosome. Journal of virology 1987 Aug;61(8):2555-8.

55. Nevins JR. Mechanism of activation of early viral transcription by the adenovirus E1A gene product. Cell 1981 Oct;26(2 Pt 2):213-20.

56. Rich DP, Couture LA, Cardoza LM, et al. Development and analysis of recombinant adenoviruses for gene therapy of cystic fibrosis. Hum Gene Ther 1993 Aug;4(4):461-76.

57. Bagchi S, Raychaudhuri P, Nevins JR. Adenovirus E1A proteins can dissociate heteromeric complexes involving the E2F transcription factor: a novel mechanism for E1A trans-activation. Cell 1990 Aug 24;62(4):659-69.

58. Mal A, Poon RY, Howe PH, Toyoshima H, Hunter T, Harter ML. Inactivation of p27Kip1 by the viral E1A oncoprotein in TGFbeta-treated cells. Nature 1996 Mar $21 ; 380(6571): 262-5$.

59. Somasundaram K, El-Deiry WS. Inhibition of p53-mediated transactivation and cell cycle arrest by E1A through its p300/CBP-interacting region. Oncogene 1997 Mar 6;14(9):1047-57.

60. Rao L, Debbas M, Sabbatini P, Hockenbery D, Korsmeyer S, White E. The adenovirus E1A proteins induce apoptosis, which is inhibited by the E1B 19-kDa and Bcl-2 proteins. Proc Natl Acad Sci U S A 1992 Aug 15;89(16):7742-6.

61. Halbert DN, Cutt JR, Shenk T. Adenovirus early region 4 encodes functions required for efficient DNA replication, late gene expression, and host cell shutoff. Journal of virology 1985;56(1):250-7.

62. Pilder S, Moore M, Logan J, Shenk T. The adenovirus E1B-55K transforming polypeptide modulates transport or cytoplasmic stabilization of viral and host cell mRNAs. Molecular and cellular biology 1986 Feb;6(2):470-6.

63. Nevels M, Rubenwolf $S$, Spruss T, Wolf $H$, Dobner T. The adenovirus E4orf6 protein can promote E1A/E1B-induced focus formation by interfering with p53 tumor suppressor function. Proc Natl Acad Sci U S A 1997 Feb 18;94(4):1206-11.

64. Field J, Gronostajski RM, Hurwitz J. Properties of the adenovirus DNA polymerase. J Biol Chem 1984 Aug 10;259(15):9487-95.

65. Challberg MD, Desiderio SV, Kelly TJ, Jr. Adenovirus DNA replication in vitro: characterization of a protein covalently linked to nascent DNA strands. Proc Natl Acad Sci U S A 1980 Sep;77(9):5105-9.

66. Burgert HG, Maryanski JL, Kvist S. "E3/19K" protein of adenovirus type 2 inhibits lysis of cytolytic $\mathrm{T}$ lymphocytes by blocking cell-surface expression of histocompatibility class I antigens. Proc Natl Acad Sci U S A 1987;84(5):1356-60.

67. Elsing A, Burgert HG. The adenovirus E3/10.4K-14.5K proteins down-modulate the apoptosis receptor Fas/Apo-1 by inducing its internalization. Proc Natl Acad Sci U S A 1998 Aug 18;95(17):10072-7.

68. Mathews MB, Shenk T. Adenovirus virus-associated RNA and translation control. Journal of virology $1991 \mathrm{Nov} ; 65(11): 5657-62$.

69. Leibowitz J, Horwitz MS. Synthesis and assembly of adenovirus polypeptides. III. Reversible inhibition of hexon assembly in adenovirus type 5 temperature-sensitive mutants. Virology 1975 Jul;66(1):10-24. 
70. Bergelson JM, Cunningham JA, Droguett G, et al. Isolation of a common receptor for Coxsackie B viruses and adenoviruses 2 and 5. Science 1997 Feb 28;275(5304):1320-3.

71. Wickham TJ, Mathias P, Cheresh DA, Nemerow GR. Integrins alpha $\vee$ beta 3 and alpha $v$ beta 5 promote adenovirus internalization but not virus attachment. Cell 1993 Apr 23;73(2):309-19.

72. Tollefson AE, Scaria A, Hermiston TW, Ryerse JS, Wold LJ, Wold WS. The adenovirus death protein (E3-11.6K) is required at very late stages of infection for efficient cell lysis and release of adenovirus from infected cells. Journal of virology 1996 Apr;70(4):2296-306.

73. Doronin K, Toth K, Kuppuswamy M, Ward P, Tollefson AE, Wold WS. Tumorspecific, replication-competent adenovirus vectors overexpressing the adenovirus death protein. Journal of virology 2000;74(13):6147-55.

74. Rauen KA, Sudilovsky D, Le JL, et al. Expression of the coxsackie adenovirus receptor in normal prostate and in primary and metastatic prostate carcinoma: potential relevance to gene therapy. Cancer Res 2002 Jul 1;62(13):3812-8.

75. Douglas JT, Rogers BE, Rosenfeld ME, Michael SI, Feng M, Curiel DT. Targeted gene delivery by tropism-modified adenoviral vectors. Nat Biotechnol 1996 Nov;14(11):1574-8.

76. Wickham TJ, Segal DM, Roelvink PW, et al. Targeted adenovirus gene transfer to endothelial and smooth muscle cells by using bispecific antibodies. Journal of virology 1996 Oct;70(10):6831-8.

77. Kraaij R, van Rijswijk AL, Oomen MH, Haisma HJ, Bangma CH. Prostate specific membrane antigen (PSMA) is a tissue-specific target for adenoviral transduction of prostate cancer in vitro. Prostate 2005 Feb 15;62(3):253-9.

78. Wickham TJ, Roelvink PW, Brough DE, Kovesdi I. Adenovirus targeted to heparan-containing receptors increases its gene delivery efficiency to multiple cell types. Nat Biotechnol 1996 Nov;14(11):1570-3.

79. Krasnykh V, Dmitriev I, Mikheeva G, Miller CR, Belousova N, Curiel DT. Characterization of an adenovirus vector containing a heterologous peptide epitope in the HI loop of the fiber knob. Journal of virology 1998 Mar;72(3):1844-52.

80. Lupold SE, Rodriguez R. Disulfide-constrained peptides that bind to the extracellular portion of the prostate-specific membrane antigen. Molecular cancer therapeutics 2004 May;3(5):597-603.

81. Shayakhmetov DM, Papayannopoulou T, Stamatoyannopoulos G, Lieber A. Efficient gene transfer into human CD34(+) cells by a retargeted adenovirus vector. Journal of virology 2000 Mar;74(6):2567-83.

82. Gaggar A, Shayakhmetov DM, Lieber A. CD46 is a cellular receptor for group B adenoviruses. Nat Med 2003 Nov;9(11):1408-12.

83. Sova $\mathrm{P}, \mathrm{Ren} \mathrm{XW}, \mathrm{Ni} \mathrm{S}$, et al. A tumor-targeted and conditionally replicating oncolytic adenovirus vector expressing TRAIL for treatment of liver metastases. Mol Ther 2004 Apr;9(4):496-509.

84. Yang Y, Nunes FA, Berencsi K, Furth EE, Gonczol E, Wilson JM. Cellular immunity to viral antigens limits E1-deleted adenoviruses for gene therapy. Proc Natl Acad Sci U S A 1994 May 10;91(10):4407-11.

85. Schiedner G, Hertel S, Johnston M, Biermann V, Dries V, Kochanek S. Variables affecting in vivo performance of high-capacity adenovirus vectors. Journal of virology 2002 Feb;76(4):1600-9.

86. Sandig V, Youil R, Bett AJ, et al. Optimization of the helper-dependent adenovirus system for production and potency in vivo. Proc Natl Acad Sci U S A 2000 Feb 1;97(3):1002-7. 
87. Parks RJ, Chen L, Anton M, Sankar U, Rudnicki MA, Graham FL. A helperdependent adenovirus vector system: removal of helper virus by Cre-mediated excision of the viral packaging signal. PNAS USA 1996;93:13565-70.

88. Fallaux FJ, Bout A, van der Velde I, et al. New helper cells and matched early region 1-deleted adenovirus vectors prevent generation of replication-competent adenoviruses. Hum Gene Ther 1998 Sep 1;9(13):1909-17.

89. Kotin RM, Siniscalco M, Samulski RJ, et al. Site-specific integration by adenoassociated virus. Proc Natl Acad Sci U S A 1990 Mar;87(6):2211-5.

90. Linden RM, Ward P, Giraud C, Winocour E, Berns KI. Site-specific integration by adeno-associated virus. Proc Natl Acad Sci U S A 1996 Oct 15;93(21):11288-94.

91. Kearns WG, Afione SA, Fulmer SB, et al. Recombinant adeno-associated virus (AAV-CFTR) vectors do not integrate in a site-specific fashion in an immortalized epithelial cell line. Gene Ther 1996 Sep;3(9):748-55.

92. Rolling F, Samulski RJ. AAV as a viral vector for human gene therapy. Generation of recombinant virus. Mol Biotechnol 1995 Feb;3(1):9-15.

93. Urabe M, Ding $\mathrm{C}$, Kotin RM. Insect cells as a factory to produce adenoassociated virus type 2 vectors. Hum Gene Ther 2002 Nov 1;13(16):1935-43.

94. Ferrari FK, Samulski T, Shenk T, Samulski RJ. Second-strand synthesis is a rate-limiting step for efficient transduction by recombinant adeno-associated virus vectors. Journal of virology 1996 May;70(5):3227-34.

95. McCarty DM, Monahan PE, Samulski RJ. Self-complementary recombinant adeno-associated virus (scAAV) vectors promote efficient transduction independently of DNA synthesis. Gene Ther 2001 Aug;8(16):1248-54 .

96. Warrington KH, Jr., Gorbatyuk OS, Harrison JK, Opie SR, Zolotukhin S, Muzyczka N. Adeno-associated virus type 2 VP2 capsid protein is nonessential and can tolerate large peptide insertions at its $\mathrm{N}$ terminus. Journal of virology 2004 Jun;78(12):6595-609.

97. Recchia A, Perani L, Sartori D, Olgiati C, Mavilio F. Site-specific integration of functional transgenes into the human genome by adeno/AAV hybrid vectors. Mol Ther 2004 Oct;10(4):660-70.

98. Brand K, Arnold W, Bartels T, et al. Liver-associated toxicity of the HSV-tk/GCV approach and adenoviral vectors. Cancer Gene Therapy 1997;4:9-16.

99. Herman JR, Adler HL, Aguilar-Cordova E, et al. In situ gene therapy for adenocarcinoma of the prostate: a phase I clinical trial. Hum Gene Ther 1999 May 1;10(7):1239-49.

100. Stamey TA, Yang N, Hay AR, McNeal JE, Feiha FS, Redwine E. ProstateSpecific antigen as a serum marker for adenocarcinoma of the prostate. $\mathrm{N}$ Engl $\mathrm{J}$ Med 1987;317:909-16.

101. Riegman PH, Vlietstra RJ, van der Korput J, Romijn JC, Trapman J. Characterization of the prostate-specific antigen gene: a novel human kallikrein-like gene. Biochemical \& Biophysical Research Communications 1989;159(1):95-102.

102. Cleutjens KB, van der Korput HA, van Eekelen CC, van Rooij HC, Faber PW, Trapman J. An androgen response element in a far upstream enhancer region is essential for high, androgen-regulated activity of the prostate- specific antigen promoter. Mol Endocrinol 1997;11(2):148-61.

103. Pang S, Dannull J, Kaboo R, et al. Identification of a positive regulatory element responsible for tissue-specific expression of prostate-specific antigen. Cancer Res 1997 Feb 1;57(3):495-9.

104. Schuur ER, Henderson GA, Kmetec LA, Miller JD, Lamparski HG, Henderson DR. Prostate-specific antigen expression is regulated by an upstream enhancer. J Biol Chem 1996;271(12):7043-51. 
105. Riegman PH, Vlietstra RJ, van der Korput JA, Brinkmann AO, Trapman J. The promoter of the prostate-specific antigen gene contains a functional androgen responsive element. Molecular Endocrinology 1991;5(12):1921-30.

106. Gotoh A, Ko SC, Shirakawa T, et al. Development of prostate-specific antigen promoter-based gene therapy for androgen-independent human prostate cancer. J Urol 1998 Jul;160(1):220-9.

107. Lu Y, Carraher J, Zhang Y, et al. Delivery of adenoviral vectors to the prostate for gene therapy. Cancer Gene Ther 1999 Jan-Feb;6(1):64-72.

108. Yeung F, Li X, Ellett J, Trapman J, Kao C, Chung LW. Regions of prostatespecific antigen (PSA) promoter confer androgen- independent expression of PSA in prostate cancer cells. J Biol Chem 2000;275(52):40846-55.

109. Wu L, Matherly J, Smallwood A, et al. Chimeric PSA enhancers exhibit augmented activity in prostate cancer gene therapy vectors. Gene Ther 2001 Sep;8(18):1416-26.

110. Latham JP, Searle PF, Mautner V, James ND. Prostate-specific antigen promoter/enhancer driven gene therapy for prostate cancer: construction and testing of a tissue-specific adenovirus vector. Cancer Res 2000 Jan 15;60(2):334-41.

111. Horoszewicz JS, Kawinski E, Murphy GP. Monoclonal antibodies to a new antigenic marker in epithelial prostatic cells and serum of prostatic cancer patients. Anticancer Res 1987;7(5B):927-35.

112. Pinto JT, Suffoletto BP, Berzin TM, et al. Prostate-specific membrane antigen: a novel folate hydrolase in human prostatic carcinoma cells. Clin Cancer Res 1996;2(9):1445-51.

113. Carter RE, Feldman AR, Coyle JT. Prostate-specific membrane antigen is a hydrolase with substrate and pharmacologic characteristics of a neuropeptidase. Proc Natl Acad Sci U S A 1996;93(2):749-53.

114. Pangalos MN, Neefs JM, Somers M, et al. Isolation and expression of novel human glutamate carboxypeptidases with $\mathrm{N}$-acetylated alpha-linked acidic dipeptidase and dipeptidyl peptidase IV activity. J Biol Chem 1999;274(13):8470-83.

115. Silver DA, Pellicer I, Fair WR, Heston WD, Cordon-Cardo C. Prostate-specific membrane antigen expression in normal and malignant human tissues. Clin Cancer Res 1997 Jan;3(1):81-5.

116. Xiao Z, Adam BL, Cazares LH, et al. Quantitation of serum prostate-specific membrane antigen by a novel protein biochip immunoassay discriminates benign from malignant prostate disease. Cancer Res 2001;61(16):6029-33.

117. Sweat SD, Pacelli A, Murphy GP, Bostwick DG. Prostate-specific membrane antigen expression is greatest in prostate adenocarcinoma and lymph node metastases. Urology 1998;52(4):637-40.

118. Wright GL, Grob BM, Haley C, et al. Upregulation of Prostate-specific membrane antigen after androgen-deprivation therapy. Urology 1996;48(2):326-34.

119. O'Keefe DS, Su SL, Bacich DJ, et al. Mapping, genomic organization and promoter analysis of the human prostate-specific membrane antigen gene. Biochim Biophys Acta 1998;1443(1-2):113-27.

120. Watt F, Martorana A, Brookes DE, et al. A tissue-specific enhancer of the prostate-specific membrane antigen gene, FOLH1. Genomics 2001;73(3):243-54.

121. Lee SJ, Lee K, Yang X, et al. NFATc1 with AP-3 site binding specificity mediates gene expression of prostate-specific-membrane-antigen. J Mol Biol $2003 \mathrm{Jul}$ 18;330(4):749-60. 
122. Uchida A, O'Keefe DS, Bacich DJ, Molloy PL, Heston WD. In vivo suicide gene therapy model using a newly discovered prostate- specific membrane antigen promoter/enhancer: a potential alternative approach to androgen deprivation therapy. Urology 2001;58(2 Suppl 1):132-9.

123. Lee SJ, Zhang Y, Lee SD, et al. Targeting prostate cancer with conditionally replicative adenovirus using PSMA enhancer. Mol Ther 2004 Dec;10(6):1051-8.

124. Lee SJ, Kim HS, Yu R, et al. Novel prostate-specific promoter derived from PSA and PSMA enhancers. Mol Ther 2002;6(3):415-21.

125. Pan LC, Price PA. The effect of transcriptional inhibitors on the bone gammacarboxyglutamic acid protein response to 1,25-dihydroxyvitamin D3 in osteosarcoma cells. J Biol Chem 1984 May 10;259(9):5844-7.

126. Jung C, Ou YC, Yeung F, Frierson HF, Jr., Kao C. Osteocalcin is incompletely spliced in non-osseous tissues. Gene 2001;271(2):143-50.

127. Wu TT, Sikes RA, Cui Q, et al. Establishing human prostate cancer cell xenografts in bone: induction of osteoblastic reaction by PSA-producing tumors in athymic and SCID/bg mice using LNCaP and lineage-derived metastatic sublines. International Journal of Cancer 1998; In press.

128. Koeneman KS, Yeung F, Chung LW. Osteomimetic properties of prostate cancer cells: a hypothesis supporting the predilection of prostate cancer metastasis and growth in the bone environment. Prostate 1999 Jun 1;39(4):246-61.

129. Bortell R, Owen TA, Bidwell JP, et al. Vitamin D-responsive protein-DNA interactions at multiple promoter regulatory elements that contribute to the level of rat osteocalcin gene expression. Proc Natl Acad Sci U S A 1992 Jul 1;89(13):6119-23.

130. Lian JB, Stein GS, Stein JL, van Wijnen AJ. Regulated expression of the bonespecific osteocalcin gene by vitamins and hormones. Vitam Horm 1999;55:443-509.

131. Banerjee C, Stein JL, Van Wijnen AJ, Frenkel B, Lian JB, Stein GS. Transforming growth factor-beta 1 responsiveness of the rat osteocalcin gene is mediated by an activator protein-1 binding site. Endocrinology 1996 May;137(5):19912000.

132. Banerjee C, Hiebert SW, Stein JL, Lian JB, Stein GS. An AML-1 consensus sequence binds an osteoblast-specific complex and transcriptionally activates the osteocalcin gene. Proc Natl Acad Sci U S A 1996 May 14;93(10):4968-73.

133. Ko SC, Cheon J, Kao C, et al. Osteocalcin promoter-based toxic gene therapy for the treatment of osteosarcoma in experimental models. Cancer Res 1996 Oct 15;56(20):4614-9.

134. Koeneman KS, Kao C, Ko SC, et al. Osteocalcin-directed gene therapy for prostate-cancer bone metastasis. World J Urol 2000;18(2):102-10.

135. Kubo H, Gardner TA, Wada Y, et al. Phase I dose escalation clinical trial of adenovirus vector carrying osteocalcin promoter-driven herpes simplex virus thymidine kinase in localized and metastatic hormone-refractory prostate cancer. Hum Gene Ther 2003 Feb 10;14(3):227-41.

136. Chiu CP, Harley CB. Replicative senescence and cell immortality: the role of telomeres and telomerase. Proc Soc Exp Biol Med 1997 Feb;214(2):99-106.

137. Kim NW, Piatyszek MA, Prowse KR, et al. Specific association of human telomerase activity with immortal cells and cancer. Science 1994 Dec 23;266(5193):2011-5.

138. Horikawa I, Cable PL, Afshari C, Barrett JC. Cloning and characterization of the promoter region of human telomerase reverse transcriptase gene. Cancer Res 1999 Feb 15;59(4):826-30. 
139. Sommerfeld HJ, Meeker AK, Piatyszek MA, Bova GS, Shay JW, Coffey DS. Telomerase activity: a prevalent marker of malignant human prostate tissue. Cancer Res 1996 Jan 1;56(1):218-22.

140. Lin HK, Wang L, Hu YC, Altuwaijri S, Chang C. Phosphorylation-dependent ubiquitylation and degradation of androgen receptor by Akt require Mdm2 E3 ligase. Embo J 2002 Aug 1;21(15):4037-48.

141. Gu J, Andreeff M, Roth JA, Fang B. hTERT promoter induces tumor-specific Bax gene expression and cell killing in syngenic mouse tumor model and prevents systemic toxicity. Gene Ther 2002 Jan;9(1):30-7.

142. Kawashima T, Kagawa S, Kobayashi N, et al. Telomerase-specific replicationselective virotherapy for human cancer. Clin Cancer Res 2004 Jan 1;10(1 Pt 1):285-92.

143. Office of Biotechnology Activities' Recombinant DNA and Gene Transfer Web Page, www4.od.nih.gov/oba/rdna.htm (Accession date: February 29, 2008).

144. Levine AJ. p53, the cellular gatekeeper for growth and division. Cell 1997 Feb 7;88(3):323-31.

145. Sherr CJ. Cancer cell cycles. Science 1996 Dec 6;274(5293):1672-7.

146. Downing SR, Russell PJ, Jackson P. Alterations of p53 are common in early stage prostate cancer. Can J Urol 2003 Aug;10(4):1924-33.

147. Eastham JA, Stapleton AM, Gousse AE, et al. Association of p53 mutations with metastatic prostate cancer. Clin Cancer Res 1995 Oct;1(10):1111-8.

148. Yang C, Cirielli C, Capogrossi MC, Passaniti A. Adenovirus-mediated Wild-Type p53 Expression Induces Apoptosis and Suppresses Tumorigenesis of Prostatic Tumor Cells. Cancer research 1995;55:4210-3.

149. Eastham JA, Hall SJ, Sehgal I, et al. In vivo gene therapy with p53 or p21 adenovirus for prostate cancer. Cancer Res 1995 Nov 15;55(22):5151-5.

150. Ko SC, Gotoh A, Thalmann GN, et al. Molecular therapy with recombinant p53 adenovirus in an androgen-independent, metastatic human prostate cancer model. Hum Gene Ther 1996 Sep 10;7(14):1683-91.

151. Eastham JA, Grafton W, Martin CM, Williams BJ. Suppression of primary tumor growth and the progression to metastasis with p53 adenovirus in human prostate cancer. J Urol 2000 Sep;164(3 Pt 1):814-9.

152. Hernandez I, Maddison LA, Wei Y, et al. Prostate-specific expression of p53(R172L) differentially regulates $\mathrm{p} 21, \mathrm{Bax}$, and $\mathrm{mdm} 2$ to inhibit prostate cancer progression and prolong survival. Mol Cancer Res 2003 Dec;1(14):1036-47.

153. Gurnani M, Lipari P, Dell J, Shi B, Nielsen LL. Adenovirus-mediated p53 gene therapy has greater efficacy when combined with chemotherapy against human head and neck, ovarian, prostate, and breast cancer. Cancer Chemother Pharmacol 1999;44(2):143-51.

154. Colletier PJ, Ashoori F, Cowen D, et al. Adenoviral-mediated p53 transgene expression sensitizes both wild-type and null p53 prostate cancer cells in vitro to radiation. Int J Radiat Oncol Biol Phys 2000 Dec 1;48(5):1507-12.

155. Sasaki R, Shirakawa T, Zhang ZJ, et al. Additional gene therapy with Ad5CMVp53 enhanced the efficacy of radiotherapy in human prostate cancer cells. Int $\mathrm{J}$ Radiat Oncol Biol Phys 2001 Dec 1;51(5):1336-45.

156. Cowen D, Salem N, Ashoori F, et al. Prostate cancer radiosensitization in vivo with adenovirus-mediated p53 gene therapy. Clin Cancer Res 2000 Nov;6(11):4402-8.

157. Pantuck AJ, Zisman A, Belldegrun AS. Gene therapy for prostate cancer at the University of California, Los Angeles: preliminary results and future directions. World $\mathrm{J}$ Urol 2000 Apr;18(2):143-7.

158. Sweeney P, Pisters LL. Ad5CMVp53 gene therapy for locally advanced prostate cancer--where do we stand? World J Urol 2000 Apr;18(2):121-4. 
159. Maki CG. Oligomerization is required for p53 to be efficiently ubiquitinated by MDM2. J Biol Chem 1999 Jun 4;274(23):16531-5.

160. Fuchs SY, Adler V, Buschmann T, Wu X, Ronai Z. Mdm2 association with p53 targets its ubiquitination. Oncogene 1998 Nov 12;17(19):2543-7.

161. Haupt $Y$, Maya R, Kazaz A, Oren M. Mdm2 promotes the rapid degradation of p53. Nature 1997 May 15;387(6630):296-9.

162. Kussie PH, Gorina S, Marechal V, et al. Structure of the MDM2 oncoprotein bound to the p53 tumor suppressor transactivation domain. Science 1996 Nov 8;274(5289):948-53.

163. Leite KR, Franco MF, Srougi M, et al. Abnormal expression of MDM2 in prostate carcinoma. Mod Pathol 2001 May;14(5):428-36.

164. Zhang Z, Li M, Wang H, Agrawal S, Zhang R. Antisense therapy targeting MDM2 oncogene in prostate cancer: Effects on proliferation, apoptosis, multiple gene expression, and chemotherapy. Proc Natl Acad Sci U S A 2003 Sep 30;100(20):1163641.

165. Wang $\mathrm{H}$, Oliver $\mathrm{P}$, Zhang Z, Agrawal S, Zhang R. Chemosensitization and radiosensitization of human cancer by antisense anti-MDM2 oligonucleotides: in vitro and in vivo activities and mechanisms. Ann N Y Acad Sci 2003 Dec;1002:217-35.

166. Zhang Z, Wang H, Prasad G, et al. Radiosensitization by antisense anti-MDM2 mixed-backbone oligonucleotide in in vitro and in vivo human cancer models. Clin Cancer Res 2004 Feb 15;10(4):1263-73.

167. Mu Z, Hachem P, Agrawal S, Pollack A. Antisense MDM2 oligonucleotides restore the apoptotic response of prostate cancer cells to androgen deprivation. Prostate 2004 Aug 1;60(3):187-96.

168. Maehama T, Dixon JE. The tumor suppressor, PTEN/MMAC1, dephosphorylates the lipid second messenger, phosphatidylinositol 3,4,5-trisphosphate. J Biol Chem 1998 May 29;273(22):13375-8.

169. Burgering BM, Kops GJ. Cell cycle and death control: long live Forkheads. Trends Biochem Sci 2002 Jul;27(7):352-60.

170. Kotelevets L, van Hengel J, Bruyneel E, Mareel M, van Roy F, Chastre E. The lipid phosphatase activity of PTEN is critical for stabilizing intercellular junctions and reverting invasiveness. J Cell Biol 2001 Dec 24;155(7):1129-35.

171. Stambolic V, Suzuki A, de la Pompa JL, et al. Negative regulation of PKB/Aktdependent cell survival by the tumor suppressor PTEN. Cell 1998 Oct 2;95(1):29-39.

172. Zundel W, Schindler C, Haas-Kogan D, et al. Loss of PTEN facilitates HIF-1mediated gene expression. Genes Dev 2000 Feb 15;14(4):391-6.

173. Davies MA, Kim SJ, Parikh NU, Dong Z, Bucana CD, Gallick GE. Adenoviralmediated expression of MMAC/PTEN inhibits proliferation and metastasis of human prostate cancer cells. Clin Cancer Res 2002 Jun;8(6):1904-14.

174. Rosser CJ, Tanaka M, Pisters LL, et al. Adenoviral-mediated PTEN transgene expression sensitizes Bcl-2-expressing prostate cancer cells to radiation. Cancer Gene Ther 2004 Apr;11(4):273-9.

175. Tanaka M, Rosser CJ, Grossman HB. PTEN gene therapy induces growth inhibition and increases efficacy of chemotherapy in prostate cancer. Cancer Detect Prev 2005;29(2):170-4.

176. Deng J, Xia W, Hung MC. Adenovirus 5 E1A-mediated tumor suppression associated with E1A-mediated apoptosis in vivo. Oncogene 1998 Oct 29;17(17):216775.

177. Bischoff JR, Kirn DH, Williams A, et al. An adenovirus mutant that replicates selectively in p53-deficient human tumor cells. Science 1996;274:373-6. 
178. Heise C, Sampson-Johannes A, Williams A, McCormick F, Von Hoff DD, Kirn DH. ONYX-015, an E1B gene-attenuated adenovirus, causes tumor-specific cytolysis and antitumoral efficacy that can be augmented by standard chemotherapeutic agents. Nat Med 1997 Jun;3(6):639-45.

179. Heise CC, Williams AM, Xue S, Propst M, Kirn DH. Intravenous administration of ONYX-015, a selectively replicating adenovirus, induces antitumoral efficacy. Cancer Res 1999;59(11):2623-8.

180. Ganly I, Kirn D, Eckhardt G, et al. A phase I study of Onyx-015, an E1B attenuated adenovirus, administered intratumorally to patients with recurrent head and neck cancer. Clin Cancer Res 2000 Mar;6(3):798-806.

181. Nemunaitis J, Khuri F, Ganly I, et al. Phase II trial of intratumoral administration of ONYX-015, a replication-selective adenovirus, in patients with refractory head and neck cancer. J Clin Oncol 2001 Jan 15;19(2):289-98.

182. Khuri FR, Nemunaitis J, Ganly I, et al. a controlled trial of intratumoral ONYX015, a selectively-replicating adenovirus, in combination with cisplatin and 5-fluorouracil in patients with recurrent head and neck cancer. Nat Med 2000 Aug;6(8):879-85.

183. Hecht JR, Bedford R, Abbruzzese JL, et al. A phase I/II trial of intratumoral endoscopic ultrasound injection of ONYX-015 with intravenous gemcitabine in unresectable pancreatic carcinoma. Clin Cancer Res 2003 Feb;9(2):555-61.

184. Morley S, MacDonald G, Kirn D, Kaye S, Brown R, Soutar D. The dl1520 virus is found preferentially in tumor tissue after direct intratumoral injection in oral carcinoma. Clin Cancer Res 2004 Jul 1;10(13):4357-62.

185. Reid TR, Freeman S, Post L, McCormick F, Sze DY. Effects of Onyx-015 among metastatic colorectal cancer patients that have failed prior treatment with 5FU/leucovorin. Cancer Gene Ther 2005 Aug;12(8):673-81.

186. Mulvihill S, Warren R, Venook A, et al. Safety and feasibility of injection with an E1B-55 kDa gene-deleted, replication-selective adenovirus (ONYX-015) into primary carcinomas of the pancreas: a phase I trial. Gene Ther 2001 Feb;8(4):308-15.

187. Nemunaitis J, Ganly I, Khuri F, et al. Selective replication and oncolysis in p53 mutant tumors with ONYX-015, an E1B-55kD gene-deleted adenovirus, in patients with advanced head and neck cancer: a phase II trial. Cancer Res 2000 Nov 15;60(22):635966.

188. Goodrum FD, Ornelles DA. p53 status does not determine outcome of E1B 55kilodalton mutant adenovirus lytic infection. Journal of virology $1998 \mathrm{Dec} ; 72(12): 9479$ 90.

189. Rothmann T, Hengstermann A, Whitaker NJ, Scheffner M, zur Hausen $H$. Replication of ONYX-015, a potential anticancer adenovirus, is independent of p53 status in tumor cells. Journal of virology 1998 Dec;72(12):9470-8.

190. Edwards SJ, Dix BR, Myers CJ, et al. Evidence that replication of the antitumor adenovirus ONYX-015 is not controlled by the p53 and p14(ARF) tumor suppressor genes. Journal of virology 2002 Dec;76(24):12483-90.

191. O'Shea CC, Johnson L, Bagus B, et al. Late viral RNA export, rather than p53 inactivation, determines ONYX-015 tumor selectivity. Cancer Cell 2004 Dec;6(6):611-23.

192. Freytag SO, Rogulski KR, Paielli DL, Gilbert JD, Kim JH. A novel three-pronged approach to kill cancer cells selectively: concomitant viral, double suicide gene, and radiotherapy. Hum Gene Ther 1998 Jun 10;9(9):1323-33.

193. Rodriguez R, Schuur ER, Lim HY, Henderson GA, Simons JW, Henderson DR. Prostate attenuated replication competent adenovirus (ARCA) CN706: a selective cytotoxic for prostate-specific antigen-positive prostate cancer cells. Cancer Res 1997;57(13):2559-63. 
194. DeWeese TL, van der Poel H, Li S, et al. A phase I trial of CV706, a replicationcompetent, PSA selective oncolytic adenovirus, for the treatment of locally recurrent prostate cancer following radiation therapy. Cancer Res 2001 Oct 15;61(20):7464-72.

195. Yu DC, Chen Y, Seng M, Dilley J, Henderson DR. The addition of adenovirus type 5 region E3 enables calydon virus 787 to eliminate distant prostate tumor xenografts. Cancer Res 1999;59(17):4200-3.

196. Dilley J, Reddy S, Ko D, et al. Oncolytic adenovirus CG7870 in combination with radiation demonstrates synergistic enhancements of antitumor efficacy without loss of specificity. Cancer Gene Ther 2005 Aug;12(8):715-22.

197. Yu DC, Chen Y, Dilley J, et al. Antitumor synergy of CV787, a prostate cancerspecific adenovirus, and paclitaxel and docetaxel. Cancer Res 2001 Jan 15;61(2):51725.

198. Matsubara S, Wada Y, Gardner TA, et al. A conditional replication-competent adenoviral vector, Ad-OC-E1a, to cotarget prostate cancer and bone stroma in an experimental model of androgen-independent prostate cancer bone metastasis. Cancer Res 2001;61(16):6012-9.

199. Hsieh CL, Yang L, Miao L, et al. A Novel Targeting Modality to Enhance Adenoviral Replication by Vitamin $\mathrm{D}(3)$ in Androgen-independent Human Prostate Cancer Cells and Tumors. Cancer Res 2002 Jun 1;62(11):3084-92.

200. Konety BR, Johnson CS, Trump DL, Getzenberg RH. Vitamin D in the prevention and treatment of prostate cancer. Semin Urol Oncol 1999 May;17(2):77-84.

201. Getzenberg RH, Light BW, Lapco PE, et al. Vitamin D inhibition of prostate adenocarcinoma growth and metastasis in the Dunning rat prostate model system. Urology 1997 Dec;50(6):999-1006.

202. Zhao XY, Feldman D. The role of vitamin D in prostate cancer. Steroids 2001 Mar-May;66(3-5):293-300.

203. Doronin K, Kuppuswamy M, Toth K, et al. Tissue-specific, tumor-selective, replication-competent adenovirus vector for cancer gene therapy. Journal of virology $2001 \mathrm{Apr} ; 75(7): 3314-24$.

204. Banerjee NS, Rivera AA, Wang M, et al. Analyses of melanoma-targeted oncolytic adenoviruses with tyrosinase enhancer/promoter-driven E1A, E4, or both in submerged cells and organotypic cultures. Molecular cancer therapeutics 2004 Apr;3(4):437-49.

205. Li X, Zhang YP, Kim HS, et al. Gene Therapy for Prostate Cancer by Controlling Adenovirus E1a and E4 Gene Expression with PSES Enhancer. Cancer Res 2005 Mar $1 ; 65(5): 1941-51$.

206. Harrison D, Sauthoff H, Heitner S, Jagirdar J, Rom WN, Hay JG. Wild-type adenovirus decreases tumor xenograft growth, but despite viral persistence complete tumor responses are rarely achieved--deletion of the viral E1b-19-kD gene increases the viral oncolytic effect. Hum Gene Ther 2001 Jul 1;12(10):1323-32.

207. Sauthoff H, Hu J, Maca C, et al. Intratumoral spread of wild-type adenovirus is limited after local injection of human xenograft tumors: virus persists and spreads systemically at late time points. Hum Gene Ther 2003 Mar 20;14(5):425-33.

208. Shen $\mathrm{BH}$, Hermiston TW. Effect of hypoxia on Ad5 infection, transgene expression and replication. Gene Ther 2005 Feb 3.

209. Lee CT, Park KH, Yanagisawa K, et al. Combination therapy with conditionally replicating adenovirus and replication defective adenovirus. Cancer Res 2004 Sep 15;64(18):6660-5. 
210. Li X, Raikwar SP, Liu YH, et al. Combination therapy of androgen-independent prostate cancer using a prostate restricted replicative adenovirus and a replicationdefective adenovirus encoding human endostatin-angiostatin fusion gene. Molecular cancer therapeutics 2006 Mar;5(3):676-84.

211. Li X, Liu YH, Lee SJ, Gardner TA, Jeng MH, Kao C. Prostate-restricted replicative adenovirus expressing human endostatin-angiostatin fusion gene exhibiting dramatic antitumor efficacy. Clin Cancer Res 2008 Jan 1;14(1):291-9.

212. Kleinberger T, Shenk T. Adenovirus E4orf4 protein binds to protein phosphatase $2 \mathrm{~A}$, and the complex down regulates $\mathrm{E} 1 \mathrm{~A}$-enhanced junB transcription. Journal of virology 1993 Dec;67(12):7556-60.

213. Huang MM, Hearing $P$. Adenovirus early region 4 encodes two gene products with redundant effects in lytic infection. Journal of virology 1989 Jun;63(6):2605-15.

214. Fang B, Eisensmith RC, Wang H, et al. Gene therapy for hemophilia B: host immunosuppression prolongs the therapeutic effect of adenovirus-mediated factor IX expression. Hum Gene Ther 1995 Aug;6(8):1039-44.

215. Kay MA, Meuse L, Gown AM, et al. Transient immunomodulation with anti-CD40 ligand antibody and CTLA4Ig enhances persistence and secondary adenovirusmediated gene transfer into mouse liver. Proc Natl Acad Sci U S A 1997 Apr 29;94(9):4686-91.

216. Poller W, Schneider-Rasp S, Liebert U, et al. Stabilization of transgene expression by incorporation of E3 region genes into an adenoviral factor IX vector and by transient anti-CD4 treatment of the host. Gene Ther 1996 Jun;3(6):521-30.

217. Kuzmin Al, Finegold MJ, Eisensmith RC. Macrophage depletion increases the safety, efficacy and persistence of adenovirus-mediated gene transfer in vivo. Gene Ther 1997 Apr;4(4):309-16.

218. Wojtowicz-Praga S. Reversal of tumor-induced immunosuppression by TGF-beta inhibitors. Invest New Drugs 2003 Feb;21(1):21-32.

219. O'Riordan CR, Lachapelle A, Delgado C, et al. PEGylation of adenovirus with retention of infectivity and protection from neutralizing antibody in vitro and in vivo. Hum Gene Ther 1999 May 20;10(8):1349-58.

220. Mok H, Palmer DJ, Ng P, Barry MA. Evaluation of polyethylene glycol modification of first-generation and helper-dependent adenoviral vectors to reduce innate immune responses. Mol Ther 2005 Jan;11(1):66-79.

221. Croyle MA, Chirmule N, Zhang Y, Wilson JM. "Stealth" adenoviruses blunt cellmediated and humoral immune responses against the virus and allow for significant gene expression upon readministration in the lung. Journal of virology 2001 May;75(10):4792-801.

222. Mesnil M, Piccoli C, Tiraby G, Willecke K, Yamasaki H. Bystander killing of cancer cells by herpes simplex virus thymidine kinase gene is mediated by connexins. Proc Natl Acad Sci U S A 1996 Mar 5;93(5):1831-5.

223. Eastham JA, Chen SH, Sehgal I, et al. Prostate cancer gene therapy: herpes simplex virus thymidine kinase gene transduction followed by ganciclovir in mouse and human prostate cancer models. Hum Gene Ther 1996 Mar 1;7(4):515-23.

224. Cheon J, Kim HK, Moon DG, Yoon DK, Cho JH, Koh SK. Adenovirus-mediated suicide-gene therapy using the herpes simplex virus thymidine kinase gene in cell and animal models of human prostate cancer: changes in tumour cell proliferative activity. BJU Int 2000 Apr;85(6):759-66.

225. Hall SJ, Mutchnik SE, Chen SH, Woo SL, Thompson TC. Adenovirus-mediated herpes simplex virus thymidine kinase gene and ganciclovir therapy leads to systemic activity against spontaneous and induced metastasis in an orthotopic mouse model of prostate cancer. Int J Cancer 1997 Jan 17;70(2):183-7. 
226. Hinata N, Shirakawa T, Terao S, et al. Progress report on phase I/II clinical trial of Ad-OC-TK plus VAL therapy for metastatic or locally recurrent prostate cancer: Initial experience at Kobe University. Int J Urol 2006 Jun;13(6):834-7.

227. Ahn M, Lee SJ, Li X, et al. Enhanced combined tumor-specific oncolysis and suicide gene therapy for prostate cancer using M6 promoter. Cancer Gene Ther 2008 Sep 5.

228. Freytag SO, Khil M, Stricker $\mathrm{H}$, et al. Phase I study of replication-competent adenovirus-mediated double suicide gene therapy for the treatment of locally recurrent prostate cancer. Cancer Res 2002 Sep 1;62(17):4968-76.

229. Freytag SO, Paielli D, Wing M, et al. Efficacy and toxicity of replicationcompetent adenovirus-mediated double suicide gene therapy in combination with radiation therapy in an orthotopic mouse prostate cancer model. Int $\mathrm{J}$ Radiat Oncol Biol Phys 2002 Nov 1;54(3):873-85.

230. Freytag SO, Stricker H, Pegg J, et al. Phase I study of replication-competent adenovirus-mediated double-suicide gene therapy in combination with conventionaldose three-dimensional conformal radiation therapy for the treatment of newly diagnosed, intermediate- to high-risk prostate cancer. Cancer Res 2003 Nov 1;63(21):7497-506.

231. Bander $\mathrm{NH}$, Yao D, Liu H, et al. MHC class I and II expression in prostate carcinoma and modulation by interferon-alpha and -gamma. Prostate 1997 Dec 1;33(4):233-9.

232. Kwon ED, Hurwitz AA, Foster BA, et al. Manipulation of T cell costimulatory and inhibitory signals for immunotherapy of prostate cancer. Proc Natl Acad Sci U S A 1997 Jul 22;94(15):8099-103.

233. Sanda MG, Ayyagari SR, Jaffee EM, et al. Demonstration of a rational strategy for human prostate cancer gene therapy. J Urol 1994 Mar;151(3):622-8.

234. Vieweg J, Rosenthal FM, Bannerji R, et al. Immunotherapy of prostate cancer in the Dunning rat model: use of cytokine gene modified tumor vaccines. Cancer Res 1994 Apr 1;54(7):1760-5.

235. Simons JW, Mikhak B, Chang JF, et al. Induction of immunity to prostate cancer antigens: results of a clinical trial of vaccination with irradiated autologous prostate tumor cells engineered to secrete granulocyte-macrophage colony-stimulating factor using ex vivo gene transfer. Cancer Res 1999 Oct 15;59(20):5160-8.

236. Simmons SJ, Tjoa BA, Rogers M, et al. GM-CSF as a systemic adjuvant in a phase II prostate cancer vaccine trial. Prostate 1999 Jun 1;39(4):291-7.

237. Simons JW, Nelson W, Nemunaitis J, et al. Phase II trials of a GM-CSF genetransduced prostate cancer cell vaccine (GVAX) in hormone refractory prostate cancer. Proc Am Soc Clin Oncol 2002;21:729A.

238. Simons JW, Higano C, Corman J, et al. A phase I/II study of high dose allogeneic GM-CSF gene-transduced prostate cancer cell line vaccine in patients with metastatic hormone-refractory prostate cancer. Proc Am Soc Clin Oncol 2003;22:166A.

239. Tjoa B, Boynton A, Kenny G, Ragde H, Misrock SL, Murphy G. Presentation of prostate tumor antigens by dendritic cells stimulates T-cell proliferation and cytotoxicity. Prostate 1996 Jan;28(1):65-9.

240. Murphy G, Tjoa B, Ragde H, Kenny G, Boynton A. Phase I clinical trial: T-cell therapy for prostate cancer using autologous dendritic cells pulsed with HLA-A0201specific peptides from prostate-specific membrane antigen. Prostate 1996 Dec;29(6):371-80.

241. Tjoa BA, Erickson SJ, Bowes VA, et al. Follow-up evaluation of prostate cancer patients infused with autologous dendritic cells pulsed with PSMA peptides. Prostate 1997 Sep 1;32(4):272-8. 
242. Tjoa BA, Simmons SJ, Elgamal A, et al. Follow-up evaluation of a phase II prostate cancer vaccine trial. Prostate 1999 Jul 1;40(2):125-9.

243. Triest JA, Grignon DJ, Cher ML, et al. Systemic interleukin 2 therapy for human prostate tumors in a nude mouse model. Clin Cancer Res 1998 Aug;4(8):2009-14.

244. Belldegrun A, Tso CL, Zisman A, et al. Interleukin 2 gene therapy for prostate cancer: phase I clinical trial and basic biology. Hum Gene Ther 2001 May 20;12(8):88392.

245. Trudel S, Trachtenberg J, Toi A, et al. A phase I trial of adenovector-mediated delivery of interleukin-2 (AdIL-2) in high-risk localized prostate cancer. Cancer Gene Ther 2003 Oct; 10(10):755-63.

246. Hu HM, Winter H, Ma J, Croft M, Urba WJ, Fox BA. CD28, TNF receptor, and IL12 are critical for CD4-independent cross-priming of therapeutic antitumor CD8+ T cells. J Immunol 2002 Nov 1;169(9):4897-904.

247. Sunamura M, Sun L, Lozonschi L, et al. The antiangiogenesis effect of interleukin 12 during early growth of human pancreatic cancer in SCID mice. Pancreas 2000 Apr;20(3):227-33.

248. He TC, Zhou S, da Costa LT, Yu J, Kinzler KW, Vogelstein B. A simplified system for generating recombinant adenoviruses. Proc Natl Acad Sci U S A 1998;95(5):2509-14.

249. Fallaux FJ, Kranenburg O, Cramer SJ, et al. Characterization of 911: a new helper cell line for the titration and propagation of early region 1-deleted adenoviral vectors. Hum Gene Ther 1996 Jan 20;7(2):215-22.

250. Sramkoski RM, Pretlow TG, 2nd, Giaconia JM, et al. A new human prostate carcinoma cell line, 22Rv1. In Vitro Cell Dev Biol Anim 1999;35(7):403-9.

251. Horoszewicz JS, Leong SS, Kawinski E, et al. LNCaP model of human prostatic carcinoma. Cancer Res 1983 Apr;43(4):1809-18.

252. Wu HC, Hsieh JT, Gleave ME, Brown NM, Pathak S, Chung LW. Derivation of androgen-independent human $\mathrm{LNCaP}$ prostatic cancer cell sublines: role of bone stromal cells. Int J Cancer 1994 May 1;57(3):406-12.

253. Kaighn ME, Narayan KS, Ohnuki Y, Lechner JF, Jones LW. Establishment and characterization of a human prostatic carcinoma cell line (PC-3). Invest Urology 1979;17:16-23.

254. Stone KR, Mickey DD, Wunderli H, Mickey GH, Paulson DF. Isolation of a human prostate carcinoma cell line (DU145). Int J Cancer 1978;21:274-81.

255. Drewinko B, Yang LY, Barlogie B, et al. Further biologic characteristics of a human carcinoembryonic antigen-producing colon carcinoma cell line. J Natl Cancer Inst $1978 \mathrm{Jul} ; 61(1): 75-83$.

256. Bubenik J, Baresova M, Viklicky V, Jakoubkova J, Sainerova H, Donner J. Established cell line of urinary bladder carcinoma (T24) containing tumour-specific antigen. Int J Cancer 1973 May;11(3):765-73.

257. Moyer MP, Manzano LA, Merriman RL, Stauffer JS, Tanzer LR. NCM460, a normal human colon mucosal epithelial cell line. In Vitro Cell Dev Biol Anim 1996 Jun;32(6):315-7.

258. Han M, Partin AW, Pound CR, Epstein JI, Walsh PC. Long-term biochemical disease-free and cancer-specific survival following anatomic radical retropubic prostatectomy. The 15-year Johns Hopkins experience. Urol Clin North Am 2001 Aug;28(3):555-65.

259. Petrylak DP, Tangen CM, Hussain $\mathrm{MH}$, et al. Docetaxel and estramustine compared with mitoxantrone and prednisone for advanced refractory prostate cancer. $\mathrm{N}$ Engl J Med 2004 Oct 7;351(15):1513-20. 
260. Tannock IF, de Wit R, Berry WR, et al. Docetaxel plus prednisone or mitoxantrone plus prednisone for advanced prostate cancer. N Engl J Med 2004 Oct 7;351(15):1502-12.

261. Wiley SR, Schooley K, Smolak PJ, et al. Identification and characterization of a new member of the TNF family that induces apoptosis. Immunity $1995 \mathrm{Dec} ; 3(6): 673-82$.

262. Pitti RM, Marsters SA, Ruppert S, Donahue CJ, Moore A, Ashkenazi A. Induction of apoptosis by Apo-2 ligand, a new member of the tumor necrosis factor cytokine family. J Biol Chem 1996 May 31;271(22):12687-90.

263. Pan G, O'Rourke K, Chinnaiyan AM, et al. The receptor for the cytotoxic ligand TRAIL. Science 1997 Apr 4;276(5309):111-3.

264. Pan G, Ni J, Wei YF, Yu G, Gentz R, Dixit VM. An antagonist decoy receptor and a death domain-containing receptor for TRAIL. Science 1997 Aug 8;277(5327):815-8.

265. Kuang AA, Diehl GE, Zhang J, Winoto A. FADD is required for DR4- and DR5mediated apoptosis: lack of trail-induced apoptosis in FADD-deficient mouse embryonic fibroblasts. J Biol Chem 2000 Aug 18;275(33):25065-8.

266. Takeda K, Hayakawa Y, Smyth MJ, et al. Involvement of tumor necrosis factorrelated apoptosis-inducing ligand in surveillance of tumor metastasis by liver natural killer cells. Nat Med 2001 Jan;7(1):94-100.

267. Degli-Esposti MA, Dougall WC, Smolak PJ, Waugh JY, Smith CA, Goodwin RG. The novel receptor TRAIL-R4 induces NF-kappaB and protects against TRAIL-mediated apoptosis, yet retains an incomplete death domain. Immunity 1997 Dec;7(6):813-20.

268. Emery JG, McDonnell P, Burke MB, et al. Osteoprotegerin is a receptor for the cytotoxic ligand TRAIL. J Biol Chem 1998 Jun 5;273(23):14363-7.

269. Nesterov A, Lu X, Johnson M, Miller GJ, Ivashchenko Y, Kraft AS. Elevated AKT activity protects the prostate cancer cell line LNCaP from TRAIL-induced apoptosis. $J$ Biol Chem 2001 Apr 6;276(14):10767-74.

270. Voelkel-Johnson C, King DL, Norris JS. Resistance of prostate cancer cells to soluble TNF-related apoptosis-inducing ligand (TRAIL/Apo2L) can be overcome by doxorubicin or adenoviral delivery of full-length TRAIL. Cancer Gene Ther 2002 Feb;9(2):164-72.

271. Shankar S, Chen X, Srivastava RK. Effects of sequential treatments with chemotherapeutic drugs followed by TRAIL on prostate cancer in vitro and in vivo. Prostate 2005 Feb 1;62(2):165-86.

272. Shankar S, Singh TR, Srivastava RK. Ionizing radiation enhances the therapeutic potential of TRAIL in prostate cancer in vitro and in vivo: Intracellular mechanisms. Prostate 2004 Sep 15;61(1):35-49.

273. Ashkenazi A, Pai RC, Fong S, et al. Safety and antitumor activity of recombinant soluble Apo2 ligand. The Journal of clinical investigation 1999 Jul;104(2):155-62.

274. Walczak H, Miller RE, Ariail K, et al. Tumoricidal activity of tumor necrosis factorrelated apoptosis-inducing ligand in vivo. Nat Med 1999 Feb;5(2):157-63.

275. Jo M, Kim TH, Seol DW, et al. Apoptosis induced in normal human hepatocytes by tumor necrosis factor-related apoptosis-inducing ligand. Nat Med 2000 May;6(5):5647.

276. Lawrence D, Shahrokh Z, Marsters S, et al. Differential hepatocyte toxicity of recombinant Apo2L/TRAIL versions. Nat Med 2001 Apr;7(4):383-5.

277. Bruder JT, Appiah A, Kirkman WM, 3rd, et al. Improved production of adenovirus vectors expressing apoptotic transgenes. Hum Gene Ther 2000 Jan 1;11(1):139-49.

278. Griffith TS, Broghammer EL. Suppression of tumor growth following intralesional therapy with TRAIL recombinant adenovirus. Mol Ther 2001 Sep;4(3):257-66. 
279. Hu B, Zhu H, Qiu S, et al. Enhanced TRAIL sensitivity by E1A expression in human cancer and normal cell lines: inhibition by adenovirus E1B19K and E3 proteins. Biochem Biophys Res Commun 2004 Dec 24;325(4):1153-62.

280. Bolla $M$, Collette $L$, Blank L, et al. Long-term results with immediate androgen suppression and external irradiation in patients with locally advanced prostate cancer (an EORTC study): a phase III randomised trial. Lancet 2002 Jul 13;360(9327):103-6.

281. D'Amico AV, Manola J, Loffredo M, Renshaw AA, DellaCroce A, Kantoff PW. 6month androgen suppression plus radiation therapy vs radiation therapy alone for patients with clinically localized prostate cancer: a randomized controlled trial. Jama 2004 Aug 18;292(7):821-7.

282. Swanson GP, Thompson IM, Tangen C, et al. Phase III Randomized Study of Adjuvant Radiation Therapy versus Oservation in Patients with Pathologic T3 Prostate Cancer (SWOG 8794). Int J Radiat Oncol Biol Phys 2005;6(Supplement 1):S1.

283. Kupelian $P$, Thames $H$, Levy L, et al. Year of treatment as independent predictor of relapse-free survival in patients with localized prostate cancer treated with definitive radiotherapy in the PSA era. Int J Radiat Oncol Biol Phys 2005 Nov 1;63(3):795-9.

284. Kupelian PA, Buchsbaum JC, Elshaikh M, Reddy CA, Zippe C, Klein EA. Factors affecting recurrence rates after prostatectomy or radiotherapy in localized prostate carcinoma patients with biopsy Gleason score 8 or above. Cancer 2002 Dec 1;95(11):2302-7.

285. Cooper JS, Ang KK. Concomitant chemotherapy and radiation therapy certainly improves local control. Int J Radiat Oncol Biol Phys 2005 Jan 1;61(1):7-9.

286. Bernier J, Domenge C, Ozsahin M, et al. Postoperative irradiation with or without concomitant chemotherapy for locally advanced head and neck cancer. $\mathrm{N}$ Engl $\mathrm{J}$ Med 2004 May 6;350(19):1945-52.

287. Sause W, Kolesar P, Taylor SI, et al. Final results of phase III trial in regionally advanced unresectable non-small cell lung cancer: Radiation Therapy Oncology Group, Eastern Cooperative Oncology Group, and Southwest Oncology Group. Chest 2000 Feb;117(2):358-64.

288. Pearcey $R$, Brundage $M$, Drouin $P$, et al. Phase III trial comparing radical radiotherapy with and without cisplatin chemotherapy in patients with advanced squamous cell cancer of the cervix. J Clin Oncol 2002 Feb 15;20(4):966-72.

289. Kumar $P$, Perrotti $M$, Weiss $R$, et al. Phase I trial of weekly docetaxel with concurrent three-dimensional conformal radiation therapy in the treatment of unfavorable localized adenocarcinoma of the prostate. J Clin Oncol 2004 May 15;22(10):1909-15.

290. Perrotti M, Doyle T, Kumar P, et al. Phase I/II trial of docetaxel and concurrent radiation therapy in localized high risk prostate cancer (AGUSG 03-10). Urol Oncol 2008 May-Jun;26(3):276-80.

291. Gong B, Almasan A. Apo2 ligand/TNF-related apoptosis-inducing ligand and death receptor 5 mediate the apoptotic signaling induced by ionizing radiation in leukemic cells. Cancer Res 2000 Oct 15;60(20):5754-60.

292. Unnithan J, Macklis RM. TRAIL induction by radiation in lymphoma patients. Cancer investigation 2004;22(4):522-5.

293. Finnberg N, Gruber JJ, Fei P, et al. DR5 knockout mice are compromised in radiation-induced apoptosis. Molecular and cellular biology 2005 Mar;25(5):2000-13.

294. Wendt J, von Haefen C, Hemmati P, Belka C, Dorken B, Daniel PT. TRAIL sensitizes for ionizing irradiation-induced apoptosis through an entirely Bax-dependent mitochondrial cell death pathway. Oncogene 2005 Jun 9;24(25):4052-64. 
295. Abou El Hassan MA, Mastenbroek DC, Gerritsen WR, Giaccone G, Kruyt FA. Overexpression of Bcl2 abrogates chemo- and radiotherapy-induced sensitisation of $\mathrm{NCl}-\mathrm{H} 460$ non-small-cell lung cancer cells to adenovirus-mediated expression of fulllength TRAIL. British journal of cancer 2004 Jul 5;91(1):171-7.

296. Wissink EH, Verbrugge I, Vink SR, et al. TRAIL enhances efficacy of radiotherapy in a p53 mutant, Bcl-2 overexpressing lymphoid malignancy. Radiother Oncol 2006 Aug;80(2):214-22.

297. Chang JY, Zhang X, Komaki R, Cheung R, Fang B. Tumor-specific apoptotic gene targeting overcomes radiation resistance in esophageal adenocarcinoma. Int $\mathrm{J}$ Radiat Oncol Biol Phys 2006 Apr 1;64(5):1482-94.

298. Zhang X, Cheung RM, Komaki R, Fang B, Chang JY. Radiotherapy sensitization by tumor-specific TRAIL gene targeting improves survival of mice bearing human nonsmall cell lung cancer. Clin Cancer Res 2005 Sep 15;11(18):6657-68.

299. Kaliberov SA, Kaliberova LN, Stockard CR, Grizzle WE, Buchsbaum DJ. Adenovirus-mediated FLT1-targeted proapoptotic gene therapy of human prostate cancer. Mol Ther 2004 Dec;10(6):1059-70.

300. Pawlik TM, Keyomarsi K. Role of cell cycle in mediating sensitivity to radiotherapy. Int J Radiat Oncol Biol Phys 2004 Jul 15;59(4):928-42.

301. van Beusechem VW, van den Doel PB, Grill J, Pinedo HM, Gerritsen WR. Conditionally replicative adenovirus expressing p53 exhibits enhanced oncolytic potency. Cancer Res 2002 Nov 1;62(21):6165-71.

302. Hallenbeck PL, Chang YN, Hay C, et al. A novel tumor-specific replicationrestricted adenoviral vector for gene therapy of hepatocellular carcinoma. Hum Gene Ther 1999;10(10):1721-33.

303. Mi J, Li ZY, Ni S, Steinwaerder D, Lieber A. Induced apoptosis supports spread of adenovirus vectors in tumors. Hum Gene Ther $2001 \mathrm{Jul}$ 1;12(10):1343-52.

304. Steinwaerder DS, Carlson CA, Otto DL, Li ZY, Ni S, Lieber A. Tumor-specific gene expression in hepatic metastases by a replication-activated adenovirus vector. Nat Med 2001 Feb;7(2):240-3.

305. Qiu S, Ruan H, Pei Z, et al. Combination of Targeting Gene-ViroTherapy with 5FU enhances antitumor efficacy in malignant colorectal carcinoma. J Interferon Cytokine Res 2004 Apr;24(4):219-30.

306. Dong F, Wang L, Davis JJ, et al. Eliminating established tumor in nu/nu nude mice by a tumor necrosis factor-alpha-related apoptosis-inducing ligand-armed oncolytic adenovirus. Clin Cancer Res 2006 Sep 1;12(17):5224-30.

307. Ren XW, Liang $M$, Meng $X$, et al. A tumor-specific conditionally replicative adenovirus vector expressing TRAIL for gene therapy of hepatocellular carcinoma. Cancer Gene Ther 2006 Feb;13(2):159-68.

308. Stevens $\mathrm{CW}$, Zeng M, Cerniglia GJ. Ionizing radiation greatly improves gene transfer efficiency in mammalian cells. Hum Gene Ther 1996 Sep 10;7(14):1727-34.

309. Zeng M, Cerniglia GJ, Eck SL, Stevens CW. High-efficiency stable gene transfer of adenovirus into mammalian cells using ionizing radiation. Hum Gene Ther 1997 Jun 10;8(9):1025-32.

310. Marini P, Jendrossek V, Durand E, Gruber C, Budach W, Belka C. Molecular requirements for the combined effects of TRAIL and ionising radiation. Radiother Oncol 2003 Aug;68(2):189-98.

311. Zhang J, Kabra NH, Cado D, Kang C, Winoto A. FADD-deficient T cells exhibit a disaccord in regulation of the cell cycle machinery. J Biol Chem 2001 Aug 10;276(32):29815-8 . 
312. Alappat EC, Feig C, Boyerinas B, et al. Phosphorylation of FADD at serine 194 by CKlalpha regulates its nonapoptotic activities. Molecular cell 2005 Aug 5;19(3):32132.

313. Scaffidi C, Volkland J, Blomberg I, Hoffmann I, Krammer PH, Peter ME. Phosphorylation of FADD/ MORT1 at serine 194 and association with a 70-kDa cell cycle-regulated protein kinase. J Immunol 2000 Feb 1;164(3):1236-42.

314. Shimada K, Nakamura M, Ishida E, Konishi N. Molecular roles of MAP kinases and FADD phosphorylation in prostate cancer. Histol Histopathol 2006 Apr;21(4):415-22.

315. Kim M, Liao J, Dowling ML, et al. TRAIL inactivates the mitotic checkpoint and potentiates death induced by microtubule-targeting agents in human cancer cells. Cancer Res 2008 May 1;68(9):3440-9. 


\title{
Curriculum Vitae
}

Juan Antonio Jiménez

\section{Education:}

M.D. (2010)

Ph.D. 2008

Indiana University School of Medicine, Indianapolis, IN. Indiana University, Indianapolis, IN. Microbiology and Immunology

B.S. 2002 Indiana University, Bloomington, IN. Microbiology

Professional Experience:

2002 - present
2005 - present
2005 (Fall)

Research Associate

Department of Urology Research Laboratory, Indiana

University Medical Center, Indianapolis, IN.

Physician Extern, Chief

Department of Emergency Medicine, Henry County Hospital,

New Castle, IN.

Associate Instructor

Department of Microbiology, Indiana University School of Medicine, Indianapolis, IN.

\section{Awards:}

2008 - present $2003-2008$

\author{
NIH Medical Scientist Training Program, 1T32GM077229-01A1. \\ $\mathrm{NIH}$ National Research Service Award Individual Fellowship, \\ NCI 1F31CA106215-01, Principle Investigator.
}

2002

\section{Publications:}

1. Jiménez, JA, Li, X, Zhang, YP, Bae, KH, Mohammadi, Y, Pandya, P, Kao, C and Gardner, TA: Antitumor activity of Ad-IU2, a prostate-specific replication-competent adenovirus encoding the apoptosis inducer, TRAIL. Cancer Gene Ther (Submitted).

2. Jiménez, JA, Mohammadi, Y, Zhang, YP, Mendonca, MS, Ko, SC, Kao, C, Gardner, TA: Radio-sensitization of androgen-independent prostate cancer by TRAIL gene therapy. (In progress).

3. Mellon MJ, Bae KH, Steding CE, Jiménez JA, Kao C and Gardner TA: Suppression of renal cell carcinoma growth and metastasis with sustained antiangiogenic gene therapy. Hum Gene Ther. 19: 487-95, 2008.

4. Mellon MJ, Ahn M, Jiménez JA, Kao C and Gardner TA: Anti-angiogenic gene therapy for metastatic renal cell carcinoma produces tumor growth suppression in an athymic nude mouse model. J Urol. 179: 737-42, 2008.

5. Ahn M, Lee SJ, Li X, Jiménez JA, Zhang YP, Bae KH, Mohammadi Y, Kao C and Gardner TA: Enhanced combined tumor-specific oncolysis and suicide gene therapy for prostate cancer using M6 promoter. Cancer Gene Ther, 2008.

6. Jiménez, JA, Li, X, Gardner, TA, and Kao, C: Gene Therapy for Advanced Prostate Cancer. Prostate Cancer: Novel Biology, Genetics and Therapeutics. $2^{\text {nd }}$ Edition. 2007. 
7. Li, X, Steding, CE, Jiménez, JA, Gardner, TA, and Kao, C: Development of prostaterestricted replication-competent adenoviral vectors. Cancer and Gene Therapy, 2007.

8. Jiménez JA, Kao $C$, Raikwar $S$ and Gardner TA: Current status of anti-angiogenesis therapy for prostate cancer. Urol Oncol. 24: 260-8, 2006.

9. Desai $P$, Jiménez JA, Kao $C$ and Gardner TA: Future innovations in treating advanced prostate cancer. Urol Clin North Am. 33: 247-72, viii, 2006.

10. Jiménez, JA, Kao, C, Lee, SJ, Jung, C, and Gardner, TA:, Gene Therapy for Prostate Cancer. Prostate Cancer: Basic Mechanisms and Therapeutic approaches. 2005.

11. Li X, Zhang YP, Kim HS, Bae KH, Stantz KM, Lee SJ, Jung C, Jiménez JA, Gardner $\mathrm{TA}$, Jeng $\mathrm{MH}$ et al:: Gene therapy for prostate cancer by controlling adenovirus E1a and E4 gene expression with PSES enhancer. Cancer Res. 65: 1941-51, 2005.

12. Gardner, TA, Jiménez, JA, Chung, LWK, and Kao, C: Antimetastatic Gene Therapy: Prostate Cancer Theory to Therapy. Cancer Gene Therapy. 2004.

13. Cho YM, Woodard GL, Dunbar M, Gocken T, Jiménez JA and Foley J: Hair-cycledependent expression of parathyroid hormone-related protein and its type I receptor: evidence for regulation at the anagen to catagen transition. J Invest Dermatol. 120: 715-27, 2003.

14. Foley J, King CS, Jiménez JA, Wysolmerski JJ and Philbrick WM: Activation of PTHrP gene expression in squamous carcinoma cell lines by mutant isoforms of the tumor suppressor p53. Oncol Res. 12: 71-81, 2000.

\section{Abstracts:}

1. American Society for Therapeutic Radiology and Oncology, Boston, MA, September 2008. Oral presentation.

2. American Society of Gene Therapy, Boston, MA, May 2008. Poster.

3. American Urologic Association, Orlando, Fl, May 2008. Poster.

4. American Society of Clinical Oncology Genitourinary Cancers Symposium, San Francisco, CA, February 2008. Poster.

5. Department of Defense Innovative Minds in Prostate Cancer Today, Atlanta, GA, September, 2007. Poster.

6. American Society of Clinical Oncology Prostate Cancer Symposium, San Franscisco, CA, February 2007. Poster.

7. International Emerging Technologies in Drug and Gene-based Therapeutics Conference, Crete, Greece, September 2005. Oral presentation. 